\title{
Neuropsychological functioning of college students with Attention Deficit Hyperactivity Disorder
}

\author{
Melanie Ann Nuszkowski \\ West Virginia University
}

Follow this and additional works at: https://researchrepository.wvu.edu/etd

\section{Recommended Citation}

Nuszkowski, Melanie Ann, "Neuropsychological functioning of college students with Attention Deficit Hyperactivity Disorder" (2008). Graduate Theses, Dissertations, and Problem Reports. 4411. https://researchrepository.wvu.edu/etd/4411

This Dissertation is protected by copyright and/or related rights. It has been brought to you by the The Research Repository @ WVU with permission from the rights-holder(s). You are free to use this Dissertation in any way that is permitted by the copyright and related rights legislation that applies to your use. For other uses you must obtain permission from the rights-holder(s) directly, unless additional rights are indicated by a Creative Commons license in the record and/ or on the work itself. This Dissertation has been accepted for inclusion in WVU Graduate Theses, Dissertations, and Problem Reports collection by an authorized administrator of The Research Repository @ WVU. For more information, please contact researchrepository@mail.wvu.edu. 
Neuropsychological Functioning of College Students with Attention Deficit Hyperactivity Disorder

Melanie Ann Nuszkowski

Dissertation submitted to the College of Human Resources and Education

at West Virginia University in partial fulfillment of the requirements for the degree of

Doctor of Philosophy

in

Counseling Psychology

Margaret Glenn, Ed.D., Chair

James Bartee, Ph.D.

Deborah J. Hendricks, Ed.D.

Larry Kontosh, Ph.D.

Daniel G. Long II, Ph.D.

Ann Richards, Ph.D.

Department of Counseling, Rehabilitation Counseling, and Counseling Psychology

Morgantown, West Virginia

2008

Keywords: ADHD, college students, neuropsychological, cognitive, executive function

Copyright 2008 Melanie A. Nuszkowski 


\title{
ABSTRACT \\ Neuropsychological Functioning of College Students with Attention Deficit \\ Hyperactivity Disorder
}

Melanie Ann Nuszkowski

\begin{abstract}
Individuals with Attention Deficit Hyperactivity Disorder (ADHD) often experience significant impairment in multiple life domains not only during childhood, but also throughout their adult lives. While there is no single litmus test for identifying ADHD, neuropsychological tests have been proposed as one means of enhancing identification of ADHD in adults. Theories of ADHD have suggested that deficits in executive functioning underlie the symptom appearance for ADHD; however, meta-analyses have suggested that non-executive functioning skills provide nearly equal discriminatory results. Given the increasing proportion of students with ADHD who are pursuing postsecondary education, the need for further research addressing the impact of ADHD on these students is paramount. This study examined the neuropsychological performance of college students with ADHD on measures of attention, response inhibition, cognition, and executive functioning. Data from 101 students at a large university in the midAtlantic region who were diagnosed with ADHD or a Learning Disorder following neuropsychological assessment were included in the study. Data analyses revealed that students with ADHD had significantly higher WAIS-III Verbal Comprehension and Perceptual Organization Index scores, comparable WAIS-III Working Memory and Processing Speed Index scores, and significantly lower IVA Full Scale Attention and Response Control Quotient scores when compared with the WAIS-III and IVA standardization samples, respectively. In addition, results indicated that students with ADHD exhibited diffuse deficits on measures of executive functioning, although results across domains were inconsistent. Clinical implications and limitations are discussed as well as suggestions for future research.
\end{abstract}




\section{ACKNOWLEDGEMENTS}

I would like to express sincere gratitude to several individuals for their support through this process. First, I would like to thank my Chair, Dr. Glenn for encouraging independence in pursuing my ideas, patiently providing the structure and parameters for making this document readable, and for helping me to maintain a focus on the clinical implications of my work. Secondly, I would like to thank Dr. Dan Long II for his unwavering enthusiasm and brilliant ideas with every aspect of this dissertation. I would also like to thank Dr. Hendricks for her humbling kindness and statistical expertise. I am especially appreciative to Dr. Bartee for his detailed review of the document, theoretical ideas, flexibility in scheduling the defense, and for the phenomenal job he does everyday in maintaining the integrity of our Counseling Psychology program. I would like to express thanks to Dr. Kontosh for his efforts in going above and beyond in helping me to address all relevant aspects of this data, particularly with hypothesis testing. Thanks also to Dr. Richards for her willingness to jump on board for the culmination of this dissertation with the departure of Dr. Stone.

I would also like to express immense thanks to T. Anne Hawkins and Tandy McClung who have graced my life with their sage wisdom, kindness, and humor. I cannot thank you enough for the tremendous influence you have had on my academic and personal development. I would also like to thank my family and friends, especially Jamie and Melissa, for their encouragement, inspiration, and laughs as I finished my doctoral training. Finally, and most importantly, I would like to thank my husband, John, for reminding me to take one step at a time and for filling every moment of my life with love! 
Table of Contents

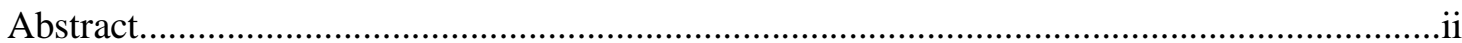

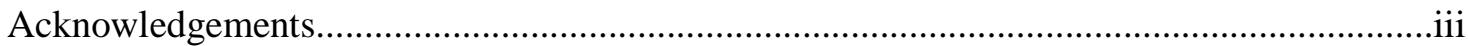

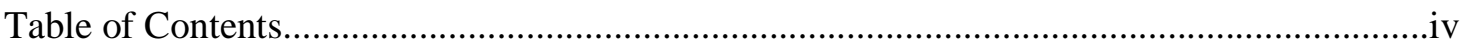

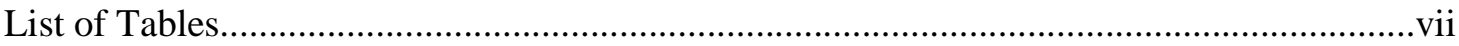

Chapter 1: Introduction and Literature Review..................................

History of Attention Deficit Hyperactivity Disorder...........................4

DSM-IV-TR Diagnostic Criteria for Attention-Deficit/Hyperactivity Disorder....5

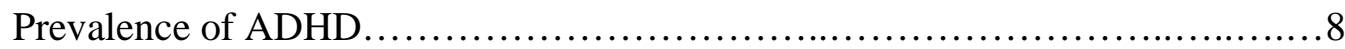

Comorbidity and Impairment in ADHD......................................12

Impairment in College Students with ADHD .................................17

Executive Functions........................................................ 18

Theoretical Models of ADHD................................................19

Brain Development and the Neurobiology of ADHD $\ldots \ldots \ldots \ldots \ldots \ldots \ldots \ldots \ldots . . \ldots \ldots$

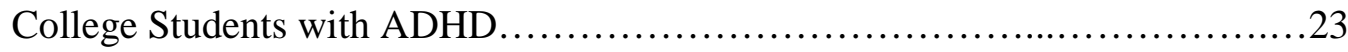

Assessment for ADHD in Adults...........................................26

Neuropsychological Functioning in Children and Adolescents with ADHD.....27

Neuropsychological Functioning in Adults with ADHD........................33

Studies Utilizing a Battery of Neuropsychological Tests.......................34

Predictive Models of ADHD...........................................44

Alternative Data Analyses and Interpretation................................46

ADHD Subtypes.......................................................... 48

Comorbidity and Specificity...........................................51

Metaanalytic Studies....................................................52 
Neuropsychological Functioning in College Students with ADHD.... .63

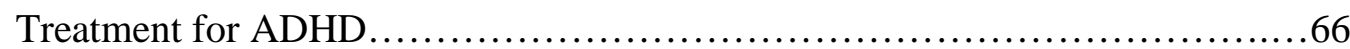

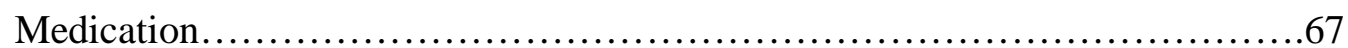

Psychotherapy......................................................68

Academic Accommodations.............................................70

Summary and Statement of the Problem...................................71

Purpose of the Current Study................................................73

Research Questions................................................73

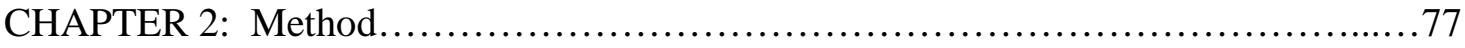

Participants........................................................ 77

Research Instruments...............................................77

ADHD Classification....................................................96

Research Design.........................................................97

CHAPTER 3: Results.......................................................... 101

Demographic Characteristics.........................................101

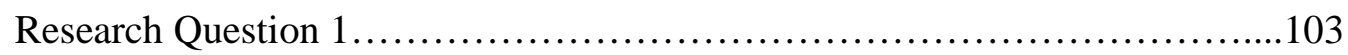

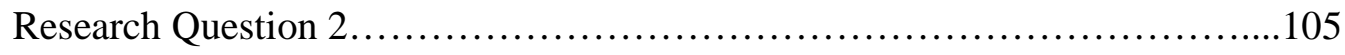

Research Question 3................................................106

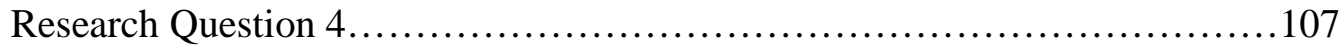

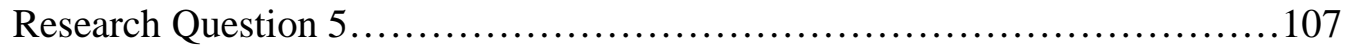

Research Question 6.................................................109

Additional Analyses...................................................111

Summary of Results..................................................112 
CHAPTER 4: Discussion........................................................ 114

Demographic Characteristics................................................ 118

Cognitive Functioning....................................................... 119

Attention and Response Inhibition..........................................122

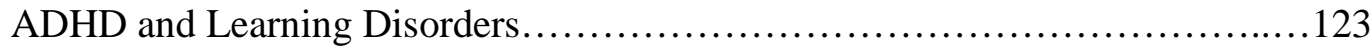

WAIS-III Index Discrepancy Scores.....................................123

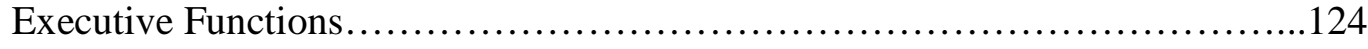

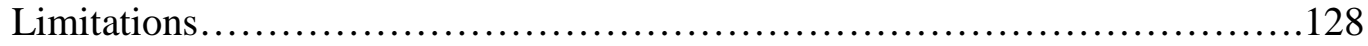

Implications for Intervention.......................................... 130

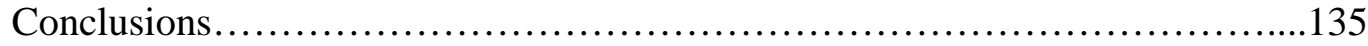

References........................................................... 138

Appendices................................................................. 153

Appendix A: ADHD/LD Assessment Screening Questionnaire.......................153

Appendix B: ADHD/LD Testing Background Information Form..................... 158 
List of Tables

Table 1. DSM-IV-TR Diagnostic Criteria for ADHD................................162

Table 2. Demographics of ADHD and ADHD/LD Participants.........................164

Table 3. Descriptive Statistics of ADHD and ADHD/LD Participants...................165

Table 4. Cognitive Functioning of Students with ADHD............................. 166

Table 5. Attention and Response Control of Students with ADHD.....................167

Table 6. WAIS-III Index Scores of Students with ADHD and ADHD/LD..............168

Table 7. Attention and Response Control of Students with ADHD and ADHD/LD.....169

Table 8. WAIS-III Index Score Discrepancy.................................... 171

Table 9. Deficits in Executive Functioning as measured by the D-KEFS ..............172

Table 10. Strengths in Executive Functioning as measured by the D-KEFS............173

Table 11. Descriptive Statistics for D-KEFS Tests of Executive Function..............174 


\section{CHAPTER 1}

Introduction and Review of the Literature

Many individuals with Attention Deficit Hyperactivity Disorder (ADHD) contend with impairment throughout their lives (Mick, Faraone, \& Biederman, 2004; Mick, Faraone, Biederman, \& Spencer, 2004; Murphy, Barkley, \& Bush, 2002; Torgersen, Gjervan, \& Rasmussen, 2006). Researchers suggest that college students with ADHD may represent a unique subset of individuals with ADHD who are less impaired (Biederman, 1993). Researchers have theorized that deficits in executive functioning underlie the symptom appearance and resulting impairment experienced by individuals with the hyperactive-impulsive and combined subtypes of ADHD (Barkley, 2006). Substantial research has addressed the relationship between executive functioning and ADHD in adults over the past decade (Hervey, Epstein, \& Curry, 2004; Schoechlin \& Engel, 2005; Woods, Lovejoy, \& Ball, 2002). Although adults with ADHD exhibit executive functioning deficits, these deficits alone have not accounted for the full symptom appearance of ADHD (Hervey, Epstein, \& Curry, 2004). Also, many of the executive functioning assessments used to date have relatively poor reliability and validity, and do not differentiate between the fundamental skills required for task completion and actual executive functioning skills (Lezak, 2002; Willcutt, Doyle, Nigg, Faraone, \& Pennington, 2005). This differentiation is particularly important in light of meta-analytic results which have suggested that fundamental skills provide nearly equal discriminatory validity for differentiating adults with and without ADHD (Boonstra et al., 2005). 
College students contending with ADHD remain an under researched population (Frazier, Youngstrom, Glutting, \& Watkins, 2007). This may be partially due to the relatively new focus on ADHD among adults. It may also be due to recent increases in the number of individuals with ADHD in the college population, following changes in the Americans with Disabilities Act (ADA) of 1990 which provided service eligibility for individuals with ADHD (Weyandt \& DuPaul, 2006).

Unfortunately, individuals with ADHD are less likely to enter college, and are even less likely to successfully complete a college education than are their non-ADHD counterparts (Barkley, 2002). Though research identifying factors that enable college students with ADHD to successfully enter and complete postsecondary education is in its infancy, research has suggested that college students with ADHD may differ from individuals with ADHD in the general population in level of education, degree of impairment, and cognitive abilities (Biederman, 1993). The development of a range of compensatory skills to adapt to academic demands (Heiligenstein, 1996; Ramsay \& Rostain, 2006; Richard, 1995; Weyandt, 2003) has also been hypothesized as a contributing factor to the success of students with ADHD. Compensatory skills and adequate performance in primary and secondary education, however, often masks ADHD. Many students with undiagnosed ADHD may experience significant academic problems for the first time upon arriving to college as their previous coping mechanisms may not continue to be as effective for the challenging demands of college life (ShawZirt, Popali-Lehane, Chaplin, \& Bergman, 2005). Thus, a substantial proportion of college students are first diagnosed with ADHD during their postsecondary education (Shaw-Zirt et al., 2005; Heiligenstein, 1996). 
The well-documented academic and psychosocial impairment among individuals with ADHD (Biederman et al., 2006; Brod, Perwien, Adler, Spencer, \& Johnston, 2005; Heiligenstein, Guenther, Levy, Savino, \& Fulwiler, 1999; Murphy, Barkley, \& Bush, 2002) brings to light the critical importance of a better understanding this disorder among college students. Better understanding their particular neuropsychological strengths and weaknesses may improve treatment planning and enable us to provide these students with support not only for academic success, but also for an improved quality of life.

Overall, individuals with ADHD appear to exhibit deficits on executive functioning tasks. However, neuropsychological profiles of adults with ADHD have varied. Researchers have hypothesized that this variation could be due to differences in samples, the diagnostic criteria used to establish a diagnosis for adult ADHD, the nature of the neuropsychological instruments used, and the difficulty in capturing the inconsistent attention that is fundamental to ADHD. Woods, Lovejoy, and Ball (2002) suggest that a battery of neuropsychological tests is needed to identify executive functioning deficits, rather than relying on a single measure. Additionally there is a tendency to assess for deficits in single domains (Hervey, Epstein, \& Curry, 2004), particularly attention or executive functioning, rather than considering that multiple pathways may better account for the heterogeneous nature of ADHD (Sonuga-Barke, 2005). Further, research regarding the neuropsychological performance of college students to date appears to suggest that college students with ADHD are less impaired than their counterparts in the general population, and may only be differentiated by a measure of continuous performance (Weyandt, Linterman, \& Rice, 1995; Weyandt, Rice, \& Linterman, 1998; Weyandt, Mitzlaff, \& Thomas, 2002; Advocat, Martino, Hill, \& 
Gouvier, 2007). This suggestion is based, however, on only a few studies with limited samples, power and generalizability, and clearly implicates the need for additional research addressing the neuropsychological performance of college students with ADHD. Thus, the purpose of this study is to examine neuropsychological functioning among college students with ADHD, using a comprehensive battery of neuropsychological measures with well established psychometric properties, to determine if a clearer picture of the neuropsychological profile exhibited by college students with ADHD can be obtained.

\section{Literature Overview}

\section{History of Attention Deficit Hyperactivity Disorder}

ADHD was first described by Still over a century ago and has been described as hyperactivity, hyperkinesis disorder of childhood, and minimal brain dysfunction (Seidman, Valera, \& Bush, 2004). The nomenclature of ADHD has undergone many changes since its inception. In the early 1900s symptoms were thought to result from a lack of moral control and failure to conform behaviors to environmental expectations (Barkley, 2006). In the 1920s, the relationship between neurological impairment and behavior disorders was noted, leading to the conception of ADHD as a neurological disorder, specifically a disorder of the frontal lobe (Wadsworth \& Harper, 2007).

In 1968, the DSM-II described children with "Hyperkinetic Reaction of Childhood" as overactive, restless, and distractible. ADHD subtypes were first presented in the DSM-III. Children were given a diagnosis of Attention Deficit Disorder, with or without Hyperactivity. Interestingly, the DSM-III-TR abandoned subtyping and instead presented a list of 14 symptoms, eight of which were required for a diagnosis. Children 
were diagnosed with ADHD, with the qualifiers of mild, moderate, or severe. The current nomenclature was first presented in 1994 with the DSM-IV. Subtypes were reintroduced as ADHD Predominantly Inattentive Type (ADHD-I), ADHD Predominantly Hyperactive-Impulsive Type (ADHD-H), ADHD Combined Type (ADHD-C), and ADHD Not Otherwise Specified (ADHD-NOS). Minor changes with the DSM-IV-TR will be presented below.

ADHD was historically conceived as a childhood disorder that was outgrown with the onset of puberty. However, by the 1970s, it became clear that a considerable proportion of children with ADHD continued to experience symptoms and associated impairment into adulthood. Doyle (2005) suggests that the move from diagnostic emphasis on motoric symptoms to cognitive functioning allowed for recognition that ADHD often persists in to adulthood.

DSM-IV-TR Diagnostic Criteria for Attention-Deficit/Hyperactivity Disorder

Please see Table 1 for the DSM-IV-TR criteria for ADHD.

In addition to the three code types presented above, ADHD-NOS is a category for disorders with prominent symptoms of inattention or hyperactivity-impulsivity that do not meet full criteria for ADHD (APA, 2000). Examples include individuals who meet criteria for ADHD, but whose age of onset is greater than seven years-old, or individuals with sub-threshold symptoms who exhibit a "behavioral pattern marked by sluggishness, daydreaming, and hypoactivity" (APA, 2000, p. 93).

Several researchers have questioned the validity of applying the diagnostic criteria for ADHD, which were developed and normed for children, to adults (Barkley, 2006; Heiligenstein, Conyers, Berns, \& Miller, 1998). Examples of criteria that do not appear 
developmentally appropriate include: often fidgets with hands or feet or squirms in seat, often leaves seat in classroom or in other situations in which remaining seated is expected, often has difficulty playing or engaging in leisure activities quietly, and often runs about or climbs excessively in situations in which it is inappropriate (in adolescents or adults, may be limited to subjective feelings of restlessness). Although a qualifier for adults has been added to the last criterion, Weyandt et al. (2003) suggest that research is needed to better understand, define, and measure the construct of subjective feelings of restlessness.

ADHD among college students, in particular, has received little scholarly attention. Some researchers have suggested that college students with ADHD may represent a unique subset of individuals with ADHD (Glutting, Youngstrom, \& Watkins, 2005; Heiligenstein, Conyers, Berns, \& Miller, 1998). Glutting et al. (2005) suggest that the outcomes obtained for children with ADHD may not apply for college students. The authors note that college students with ADHD are likely to have higher ability levels, better academic success in primary and secondary school, and greater compensatory skills than individuals with ADHD from the general population. The authors further suggest that college students with ADHD experience different stressors, namely adapting to the academic demands that accompany postsecondary education, than adults with ADHD who do not pursue a college education. Biederman et al. (1993) found that when compared to the general population, college students with ADHD have differed in cognitive abilities, degree of impairment, and education level.

ADHD criterion thresholds and the age of onset criterion have also been challenged. Heiligenstein et al. (1998) examined ADHD criteria thresholds in college 
students. A total of 448 students completed a modified version of the ADHD Rating Scale. First, the researchers found that the hyperactivity and total item scores significantly declined with increasing age, a finding that is consistent with previous reports (Murphy \& Barkley, 1996). The researchers also determined that a cutoff score of four for ADHD symptoms of hyperactivity-impulsivity and inattention would be sufficient to distinguish a college student with ADHD from the norm group. They suggest that this study provides preliminary evidence that the DSM-IV thresholds for ADHD symptoms may be too high when applied to college students. They argue that this is expected given that the DSM-IV criteria were developed and normed for children who display these behaviors more frequently. The results of this study question the applicability of the DSM-IV criteria to college students. Murphy and Barkley (1996) also found that four to five symptoms of ADHD are enough to set an adult with ADHD apart from the rest of population.

Gordon (2000), however, commented on the limitations of the Heiligenstein et al. (1998) study. In particular, he questioned the validity of an ADHD diagnosis given that several of the DSM-IV diagnostic criteria were not fully considered. He also questioned Heiligenstein's exuberance in suggesting a special subtype of ADHD for college students, based on the results of one study. Heiligenstein's response to Gordon, which reflected concern about the applicability of the DSM-IV's criteria for diagnosing ADHD in adults, is a concern shared by other researchers (Murphy, 2002; Murphy \& Barkley, 1996) and an area in need of clarification.

The age of onset criterion as defined in the DSM-IV-TR is also of concern as applied to both children and adults. Applegate et al. (1997) found that requiring age of 
onset before seven years actually reduced the accuracy of identification of currently impaired boys and girls. For adults, research has suggested that the age criterion of seven years is too young, particularly when requesting retrospective reports from individuals who may not recall their behaviors at this young age. Barkley and Biederman (1997) suggest the age of onset criterion for symptom onset or impairment should be very generously interpreted as occurring sometime in childhood until empirical justification for a precise age of onset criterion is established. Some researchers have suggested that a cut off for symptoms and impairment of approximately 12 years is more appropriate (Stavro, Ettenhofer, \& Nigg, 2007). In fact, this adjusted age cut off is now commonly used in studies of adult ADHD (Murphy, Barkley, \& Bush, 2001; Nigg et al., 2005). Despite concerns regarding the appropriateness of DSM-IV-TR diagnostic criteria for the diagnosis of ADHD among adults, the DSM-IV-TR remains the current standard for use in research and clinical practice (Hervey, Epstein, \& Curry, 2004). It is hoped that the DSM-V will provide improved diagnostic considerations for ADHD as experienced in adulthood.

Prevalence of $A D H D$

According to the DSM-IV-TR, the prevalence of ADHD has been estimated at $3 \%$ to $7 \%$ in school-age children (APA, 2000). Though prevalence rates vary due to the diagnostic definitions used, ADHD is thought to occur in approximately $4.7 \%$ of adults in the United States (Adler, 2006). Murphy \& Barkley (1996) evaluated the frequency of self-reported DSM-IV items from a large sample of 720 adults who were renewing their driver's licenses. Prevalence rates of $0.9 \%$ for ADHD-C, $2.5 \%$ for ADHD-H, and $1.3 \%$ for ADHD-I, with a total prevalence rate of $4.7 \%$ for all ADHD subtypes was found. The 
prevalence estimates did not, however, take into account all of the required criteria for a DSM-IV diagnosis of ADHD.

Several researchers have suggested that the majority of children diagnosed with ADHD display symptoms into adulthood, though the expression of symptoms often changes over time (Millstein, Wilens, Biederman, \& Spencer, 1997; Weiss, Murray, \& Weiss, 2002; Wilens, Biederman, \& Spencer, 2002). While hyperactive and impulsive symptoms attenuate for most individuals during late adolescence and adulthood, other adults may retain partial symptoms into mid-adulthood (APA, 2000). Millstein et al. (1997) found that symptoms of inattention remained prominent in up to $90 \%$ of clinically referred adults. In a national comorbidity study, Kessler (2006) found a 36\% persistence rate of ADHD in adults who were first diagnosed with ADHD in childhood. In reviewing follow-up studies, Barkley (2006) concluded that a consistent $30 \%$ to $80 \%$ of children diagnosed with ADHD continue to demonstrate persisting symptoms of ADHD with concurrent impairment into adolescence and young adulthood. Barkley (2006) suggests that more recent studies using more contemporary and rigorous diagnostic criteria consistently find higher rates of ADHD symptoms persistence than earlier, less methodologically rigorous studies have found.

Relatively little is known about the prevalence of ADHD among the college population. However, the recent interest in the persistence of ADHD into adulthood and the recent increase in the number of individuals with ADHD who are able to successfully enter postsecondary education may provide for better prevalence estimates. Wolf (2001) suggests that the past 25 years of special education and disability laws have enabled increasing numbers of students with disabilities to attend and graduate from college. The 
Individuals with Disabilities Act of 1975, Section 504 of the Rehabilitation Act of 1973, and the American with Disabilities Act enacted by Congress in 1990 mandate services and accommodations for students with disabilities. Wolf (2001) described ADHD, learning, and psychiatric disorders among students as "hidden disabilities." Henderson (1999) provided estimates on disabilities among a national survey of freshman entering colleges in the United States. Henderson indicated that in 1978, approximately $3 \%$ of freshman entering college reported a disability. In 1998, this number had tripled to more than $9 \%$. Approximately $42 \%$ of the students reported learning disorders, and $22 \%$ percent reported "other" disabilities, a category including ADHD, psychiatric disabilities, and individuals with multiple disabilities. Unfortunately, the exact number of students reporting ADHD can not be determined from this survey.

The majority of data on prevalence estimates of ADHD are derived from selfreport. Overall, estimates suggest that ADHD affects approximately $3 \%$ to $4 \%$ of the college student population in the United States (DuPaul et al., 2001; Heiligenstein, Conyers, Berns, \& Smith, 1998; Weyandt, Linterman, \& Rice, 1995). Weyandt et al. (1995) investigated the prevalence of self-reported ADHD symptoms in 770 college students (323 males, 447 females) using the Adult Rating Scale (ARS) and the Wender Utah Rating Scale (WURS). The authors considered a score of 1.5 standard deviations above the mean as indicative of having a high level of ADHD symptoms.

Results revealed that $7 \%$ of the students reported high ADHD symptoms on the ARS, indicative of current ADHD symptoms. A total of 8.7\% reported high ADHD symptoms on the WURS, a measure of ADHD in childhood. Only $2.5 \%$ reported high symptoms on both instruments. These rates are based, however, solely on self-report and 
may not reflect the prevalence of students meeting the full DSM-IV criteria for a diagnosis of ADHD. Similarly, Heiligenstein, Conyers, Berns, and Smith (1998) collected self-reports of DSM-IV symptoms from 448 college students in Wisconsin using the ADHD Rating Scale. They found an overall prevalence rate of $4 \%$ for all subtypes, with prevalence rates of $2.2 \%$ for ADHD-I, and $0.9 \%$ for both ADHD-C and ADHD-H.

In addition to estimates from the United States, DuPaul et al. (2001) provide data regarding the structure and prevalence of ADHD symptoms in 1209 university students from three countries: Italy, New Zealand, and the United States. Participants completed the Young Adult Rating Scale (YARS), a 24 item questionnaire constructed by the investigators, with 17 items directly from the DSM-IV symptom list and an additional 8 items which address difficulties that college students may encounter. The prevalence rates for ADHD by gender were: $2.9 \%$ for men and $3.9 \%$ for women in the United States; $7.4 \%$ for men and $0 \%$ for women in Italy; and $8.1 \%$ for men and $1.7 \%$ for women in New Zealand. The overall prevalence rate for male and female students in the US was approximately 3.5\%. Prevalence rates for gender and subtype for the US sample were: ADHD-I (0.7\% men, 0.8\% women), ADHD-H (2.0\% men, $2.3 \%$ women), and ADHD-C (0.2\% men, $0.8 \%$ women).

The study found no significant gender differences for self-reported symptoms of inattention or hyperactivity-impulsivity among the US sample. This finding differs from the vast literature attesting to a higher prevalence of ADHD among boys than girls (Barkley, 2006; Carlson \& Mann, 2000). One could speculate that fewer males with ADHD gain entrance to college, or that the gender ratios for boys and girls are skewed by 
referral patterns. The child literature has substantiated a higher prevalence of ADHD-H and ADHD-C among males (Gaub \& Carlson, 1997). The behaviors exhibited by these two subtypes are linked to more externalizing behaviors that are likely to attract attention. Unfortunately, it is suspected that many young females with ADHD go undetected. Another possibility is that this is a nonreferred sample of college students. This sampling strategy may improve the detection of otherwise overlooked individuals with ADHD. A limitation to the DuPaul et al. (2001) study, however, is the self-report nature of ADHD symptoms that have not been fully substantiated by other diagnostic criteria.

The prevalence rates of ADHD in college students, $4 \%$ from the Heiligenstein et al. (1998) study and 3.5\% from the DuPaul et al. (2001) study, appear to fall just below the $4.7 \%$ prevalence found in the general population. These results are promising, suggesting that people with ADHD are entering college at much the same rate as the general population. Research on adults with ADHD has shown that they are significantly less likely to graduate than adults without ADHD (Biederman et al., 2006). It is hoped that additional research and concurrent support and accommodations will enable more students with ADHD to successfully complete postsecondary education. Prevalence rates for the ADHD subtypes found among college students differed somewhat between the studies. In the DuPaul et al. study, ADHD-H was the most prominent subtype, where ADHD-I was most prominent in the Heiligenstein et al. study. This disparity may be due to sample and diagnostic assessment differences. Additional research is needed to clarify the nature and extent of ADHD among the college student population. Comorbidity and Impairment in Children, Adolescents, and Adults with ADHD 
High rates of comorbidity (Weiss, Murray, \& Weiss, 2002; Wilens, Biederman, \& Spencer, 2002) and significant functional impairment (Biederman et al., 1996; Biederman et al., 2006; Brod, Perwien, Adler, Spencer, \& Johnston, 2005; Stavro, Ettenhofer, \& Nigg, 2007; Torgersen, Gjervan, \& Rasmussen, 2006) have been found among both children and adults with ADHD. ADHD in childhood is associated with conduct disorder, oppositional defiant disorder, learning disorders, mood and anxiety disorders, and academic impairment (Biederman et al., 1996). In addition, difficulty with sustained attention is often interpreted by parents and teachers as "...indicating laziness, a poor sense of responsibility, and oppositional behavior" (APA, 2000, p. 88). This often exacerbates parent-child discord and can complicate familial dynamics (APA, 2000).

Biederman et al. (1996) conducted a prospective 1-year and 4-year follow-up study of 260 males, ages 6 to 17, with and without ADHD (140 with ADHD, 120 normal controls). They found that children with ADHD were significantly more likely to have enuresis, or a conduct, oppositional defiant, mood, anxiety, or tic disorder. Among males with ADHD, the rates of disruptive behavior, anxiety, mood, substance use, and language disorders increased significantly from baseline to follow-up assessment, whereas among the control males, only oppositional defiant and substance use disorders increased. Males with ADHD also performed lower on measures of intellectual functioning and academic achievement than did non-ADHD males at follow-up. On all assessed subscales of the WISC-R, males with ADHD had significantly more impaired scores than their control counterparts. Males with ADHD also had significantly more impaired reading and arithmetic achievement scores and significantly higher rates of learning disabilities and school dysfunction than non-ADHD males. During the follow-up period, rates of 
arithmetic disability, repeated grades, need for extra help, and placement in special classes significantly increased among males with ADHD. Impaired psychosocial functioning, including school behavior, spare-time activities, spare-time problems, problems with peers, siblings, and parents, and family functioning was also prominent among males with ADHD. Of particular interest is the finding that males with ADHD and psychiatric comorbidity did not differ significantly from males with ADHD and no comorbidity on school dysfunction, achievement, and measures of intelligence. This suggests that the cognitive deficits in males with ADHD may result from ADHD rather than comorbid disorders. In summary, males with ADHD were at high risk of experiencing a wide range of functional impairment and adverse outcomes. Additional research will be needed to determine if these results are generalizable for women.

Adults with ADHD share comorbidity with antisocial personality, mood, anxiety, conduct, and substance use disorders (Weiss, Murray, \& Weiss, 2002; Young, Toone, \& Tyson, 2003). In fact, comorbidity appears to be the rule rather than an exception. In a clinically referred group of adults, Millstein, Wilens, Biederman, \& Spencer (1997) found that $56 \%$ of their sample had four or more psychiatric comorbidities. Males were more likely to report conduct disorder, antisocial disorder, alcohol and drug dependence and stuttering, where females had higher rates of major depression, bulimia, and simple phobia. Though comorbidities differed by gender, no significant gender differences were reported in current ADHD symptomology.

Substantial impairment was also identified in a sample of 160 young adults, aged 17 to 28 years, 60 of whom were diagnosed with ADHD-C, 36 with ADHD-I, and 64 normal controls. Individuals with ADHD-C and ADHD-I were significantly more likely 
to have dysthymia, alcohol and cannabis abuse, and learning disorders when compared to normal controls. Young adults with ADHD-C were also more likely than both of the other groups to have Oppositional Defiant Disorder. Similarly, on the Symptom Checklist 90 - Revised, individuals with both subtypes of ADHD reported significantly more maladjustment in the domains of somatization, obsessive-compulsive, interpersonal sensitivity, depression, anxiety and psychoticism. Symptoms of impulsivity and paranoid ideation, as well as an arrest history were more significant among young adults with ADHD-C than both of the other groups.

The achievement problems exhibited by children and young adults have been confirmed by a metaanalysis. Frazier, Youngstrom, Glutting, and Watkins (2007) conducted a metaanalysis inclusive of 72 studies, 54 involving children, 7 with adolescents, 4 with college students, and 7 with adult populations. A medium effect size revealed that individuals with ADHD performed significantly poorer on overall measures of achievement than did the control group. The authors also converted all of the effect sizes into Wechsler metric, with results ranging from a standard score of 88 to 93 . They suggest that based on these results, clinicians can expect individuals with ADHD to obtain a standard score of 89 on measures of achievement.

The difficulties associated with ADHD frequently persist into adolescence and adulthood. Biederman et al. (2006) surveyed functional impairments in a non-referred sample of 1001 adults. Participants included 500 adults who self-reported an ADHD diagnosis and 501 non-ADHD adults drawn from a national sample representative of the US population. When compared with non-ADHD adults, adults with ADHD had significantly lower grades, lower income, higher divorce rates, and were more likely to 
engage in anti-social and addictive behaviors. Adults with ADHD were also significantly less likely to attain education, graduate from high-school, obtain a college degree, to be employed, to perceive that they fit in with peers, to have a good relationship with their parents, and to express a positive self-image or optimism. These results suggest that adults with ADHD have significant impairment in multiple life domains. Limitations to the study, however, include the self-report nature of the ADHD diagnosis and sampling differences between those who did and did not choose to complete the survey.

Similar results were found in a community recruited sample of 195 adults. Stavro, Ettenhofer, and Nigg (2007) found that when compared with non-ADHD adults, adults with ADHD had fewer years of education, attained lower incomes, and were more likely to be in the workforce or seeking work, and attending community or technical college. They were also less likely to attend full time university and when doing so had lower grade point averages.

Brod, Perwien, Adler, Spencer, and Johnston (2005) collected data from experts, published literature, and adults with ADHD to determine the links between ADHD symptoms, impairment, and quality of life. Results revealed that ADHD symptoms negatively impact multiple domains of functioning among adults with the disorder, including: work, daily activities, relationships, and physical and psychological wellbeing. The authors state that, "Furthermore, the symptoms and associated impairments tend to interact with others creates a cascading effect such that ADHD is not just a mental health condition but also a way of life" (Brod et al., 2005, p. 62). Adults with ADHD who have not been treated for the disorder are particularly impaired (Torgersen, Gjervan, \& Rasmussen, 2006). 


\section{Impairment in College Students with ADHD}

The significant impairment among children and adults with ADHD suggests that college students are also likely to experience impairment. Frazier, Youngstrom, Glutting, and Watkins (2007) investigated the one-year predictive validity of ADHD ratings in forecasting college achievement for 380 students. The College ADHD Response Evaluation was completed by the participants and their parents. Logistic regression revealed that as a set, the 5 predictors (student-rated inattentiveness, hyperactivity, and impulsivity; parent-rated inattentiveness and hyperactivity) distinguished college students on academic probation from those with average to above-average achievement. More specifically, student-rated inattentiveness and parent-rated inattentiveness made significant contributions to the prediction of academic success. These results support the importance of attentiveness in the prediction of academic achievement.

Using a retrospective chart review, Heiligenstein, Guenther, Levy, Savino, and Fulwiler (1999) investigated the psychological and academic impairments in college students presenting at the university counseling service. A total of 54 charts met the inclusion criteria of a documented diagnosis of ADHD or a request for career assessment, and with no active comorbidity. Twenty-six college students composed the ADHD group. A diagnosis of ADHD was determined by a Brown Adult ADHD Rating Scale score greater than 50, reported childhood symptoms of ADHD, and a Test of Variables of Attention (TOVA) consistent with ADHD. The control group consisted of 28 students who presented for career assessment and did not return beyond the career interpretation session. The students with ADHD reported significantly lower mean GPAs and were more likely to be on academic probation. Students with ADHD did not, however, report 
more psychological problems than did the control group. The absence of active comorbidity was part of the inclusion criteria for the control group, thus bringing into question the validity of the last finding. Also, psychological problems were identified from a questionnaire students fill out when presenting at the counseling center. Students may have been less willing to disclose their concerns on this form. Additional research is needed to clarify whether comorbidity and impairment among college students with ADHD is comparable to that found in the general population of adults with ADHD. A review of theoretical models of ADHD and the role of executive functions may provide a basis for better understanding the nature of ADHD among college students with ADHD.

\section{Executive Functions}

Intelligence has been defined as the "capacity of the individual to act purposefully, to think rationally, and to deal effectively with his environment" (Wechsler, 1997, p. 1). Executive functioning has been defined in numerous different ways. Barkley (2006, p. 305) defines executive functions as "a specific class of self-directed actions by the individual that are being used for self-regulation toward the future." Welsh and Pennington (1988, p. 201-202) define executive function as

... the ability to maintain an appropriate problem-solving set for attainment of a future goal. This set can involve one or more of the following: (a) an intention to inhibit a response or to defer it to a later more appropriate time, (b) a strategic plan of action sequences, and (c) a mental representation of the task, including the relevant stimulus information encoded in memory and the desired future goalstate. 
Nigg (2006, p. 106) states that "executive functioning" has begun to be replaced in the research literature by "cognitive control." He uses the terms interchangeably and describes them as functions that “... involve deliberate control of behavior, thought, or emotion. Such control is goal-directed and effortful, in that it requires mental resources." The concept of executive functions is also related to the cognitive psychology notion of a limited-capacity central processing system (Welsh and Pennington, 1988). Executive functions have been defined in many ways; however, the meaning of executive functioning is extremely ambiguous (Wolf \& Wasserstein, 2001). This creates difficulty in making comparisons across studies that define executive functioning differently.

\section{Theoretical Models of ADHD}

A comprehensive neuropsychological model of ADHD has yet to be proposed (Sagvolden, Johansen, Aase, \& Russell, 2005). This may relate to the tendency for traditional theories of ADHD to focus on single core dysfunctions in search of a "grand theory" of ADHD (Sonuga-Barke, 2002). Further, "The heterogeneity of its clinical expression and its multi-factorally determined aetiology makes achieving the sort of theoretical unity required by such models of AD/HD unlikely" (Sonuga-Barke, 2002, p. 29). The two primary theoretical models proposed are cognitive dysfunction models (Sonuga-Barke, 2005) and motivational dysfunction models (Sagvolden et al., 1998).

Cognitive dysfunction models posit that executive dysfunction results from deficient inhibitory control. Barkley (2006) provides a detailed elaboration of this theory of ADHD. He suggests that problems with behavioral inhibition interfere with four executive functions: working memory, self-regulation of affect/motivation/arousal, internalization of speech, and reconstitution. Barkley posits that deficits in the above four 
EFs lead to problems with motor control, fluency, and/or syntax. Concern about this theory emphasizes the finding that children and adults tend to perform poorly across nearly the entire range of neuropsychological measures. Thus, theories of ADHD that predict a broad array of neuropsychological deficits are likely to be supported by both current and future studies (Hervey, Epstein, \& Curry, 2004). Although neuropsychological research has consistently found a moderate association between executive dysfunction and ADHD, executive deficits alone do not appear to fully account for the expression of the disorder. Research addressing the role of inhibitory deficits and executive functioning will be elaborated in the following text.

Motivational dysfunction or delay aversion models suggest that ADHD is a functional expression of an underlying motivational style, in which children with ADHD are motivated to escape or avoid delay. The functional expression of this style is represented by ADHD behaviors such as inattention and hyperactivity. In more specific terms, the delay aversion hypothesis is defined as

... ADHD is the outcome of neurobiologic impairment in the power and efficiency with which the contingency between present action and future rewards is signaled. This leads to a reduction in the control exerted by future rewards on current behavior, a diminution in their "value," and an increase in the extent to which they are discounted ... (Sonuga-Barke, 2005, p. 1233).

Recently, there has been a transition from models positing a single core deficit to multiple-deficit models (Sonuga-Barke, 2005). This shift has encouraged the emergence of theoretical models that acknowledge and attempt to account for the neuropsychological heterogeneity of ADHD (Castellanos \& Tannock, 2002; Nigg et al. 
2005; Sonuga-Bark 2005). Sonuga-Barke (2005), for example, describes a dual pathway hypothesis of ADHD inclusive of tenets from both cognitive and motivational dysfunction models. This model suggests that “ ... alterations within the executive circuit modulated by mesocortical dopamine and the reward circuit modulated by mesolimbic dopamine constitute more or less discrete neuropsychologic bases for dissociable psychological processes leading to executive/inhibitory deficits and delay aversion, respectively" (Sonuga-Barke, 2005, p. 1233). Research supporting this theory found that measures of inhibitory control and delay aversion exhibit modest predictive validity when used individually, but exhibited excellent discriminate validity when used in combination (Solanto et al., 2001).

Although ADHD has aroused vast scientific interest, it remains one of the least well characterized mental disorders (Sonuga-Barke, 2002). In regards to the two primary theories, research with ADHD-C children has confirmed that both delay aversion and poor inhibitory control are core, but unrelated characteristics (Solanto et al., 2001). This finding highlights the difficulty in individually substantiating either the cognitive or motivational theory of ADHD. Though promising, this finding not only awaits replication with the other subtypes of ADHD, but also with the adult population. Given the lack of a well defined and empirically supported theory of ADHD, the results of the current study will be explored in light of literature in the field, including the neurobiology of ADHD and the role of brain development.

\section{Brain Development and the Neurobiology of ADHD}

Though the neurobiology of ADHD is not completely understood, research suggests that dysfunction in dopaminergic and noradrenergic systems underlie the 
disorder (Spencer, Biederman, \& Mick, 2007). Spencer et al. (2007, p. 78) indicate that "Prefrontal hypotheses in ADHD have primarily involved the dorsolateral prefrontal cortex, associated with organizational, planning, working memory, and attentional dysfunctions, and orbital lesions associated with social disinhibition and impulse control." Spencer, Biederman, and Mick (2007) suggest that brain imaging studies implicate frontosubcortical pathway dysfunction in individuals with ADHD. Spencer et al. (2007) indicate that these subcortical structures are part of the neural circuitry responsible for behavioral inhibition, executive functioning, motor control, and reward pathway modulation. Researchers suggest that medication for ADHD may be effective due to its impact on dopaminergic and/or noradrenergic pathways, which improve frontal inhibition (Faraone, 2004; Spencer et al., 2007).

The impact of brain development on ADHD at different ages is pertinent as brain maturation may partially explain changes in symptom appearance from childhood to adulthood. The most common findings from structural imaging studies of individuals with ADHD is smaller volumes in the frontal cortex, cerebellum, and subcortical structures. Castellanos et al. (2002) found smaller total cerebral brain volumes from childhood through adolescence. Castellanos et al. (2002) report that ADHD is associated with an approximate 3\% decrease in volume throughout the brain. They further found that "developmental curves for individuals with ADHD, although significantly lower, were essentially parallel to curves for healthy controls, with the exception of the caudate nucleus" (Castellanos et al., 2002, p. 1746). Castellanos et al. (2002, p. 1747) conclude that " ... fundamental developmental processes active during late childhood and 
adolescence are essentially healthy in ADHD, and that neuropsychiatric symptoms appear to reflect fixed earlier neurobiological insults or abnormalities."

The cerebellum and corpus callosum have also been implicated in the pathophysiology of ADHD as the cerebellum impacts cognitive functioning and the corpus callosum connects the two brain hemispheres (Castellanos, Lee, Sharp, et al., 2002). The authors suggest that size variations in these regions may decrease communication between the hemispheres, thus accounting for some of the cognitive and behavioral symptoms of ADHD (Castellanos, Lee, Sharp, et al., 2002).

Overall, studies suggest that the prefrontal cortex and frontal-subcortical networks are brain regions linked to executive functions and ADHD (Nigg, 2006). Neuroimaging studies have shown that these key regions remain underdeveloped in individuals with ADHD as late as adolescence (Castellanos, Lee, Sharp, et al., 2002; Seidman, Valera, \& Bush, 2004). Metabolic and structural differences associated with ADHD have also been shown to persist into adulthood (Hesslinger et al., 2002). In reviewing 29 neuropsychological and brain imaging studies addressing the etiology and pathophysiology of adults with ADHD, Faraone (2004, p. 37) concluded that, "These studies implicate frontosubcortical neural circuits in the pathophysiology of the disorder but also point to more widespread deficits, suggesting that any theory of the pathophysiology of ADHD must provide a model for understanding widely distributed brain dysfunction." A question that remains unknown is whether ADHD symptoms attenuate as these regions mature (Carr, Nigg, \& Henderson, 2006). 
In a longitudinal study of young adult outcomes of children with ADHD, only $22 \%$ of the sample entered college as compared with $78 \%$ of high school graduates overall, and of these students only 5\% eventually graduated (Barkley, 2002). The question of what is unique or different about the subpopulation of individuals with ADHD who are academically successful has yet to be answered. Research addressing the identification of factors that enable college students with ADHD to successfully enter and complete postsecondary education is clearly warranted.

Some college students with ADHD enter college with a diagnosis of ADHD, while others remain undetected and undiagnosed throughout their primary and secondary education. Though many students with ADHD exhibit academic impairment or behavioral problems which warrant early assessment and intervention, others do not. In fact, researchers have found that a substantial proportion of college students are first diagnosed with ADHD during college (Shaw-Zirt et al., 2005; Heiligenstein, 1996). College students with ADHD may differ from individuals with ADHD in the general population in level of education, degree of impairment, and cognitive abilities (Biederman, 1993). Researchers have suggested that college students with ADHD have developed a range of compensatory skills to adapt to academic demands (Heiligenstein, 1996; Ramsay \& Rostain, 2006; Richard, 1995; Weyandt, 2003). Thus, many of these students performed adequately during their primary and secondary education. Students whose performance does not fall below grade level, and students who do not display disruptive behavior problems are unlikely to meet criteria for school assessment (Heiligenstein, 1996; Richard, 1995). Further, individuals with sluggish cognitive tempo (SCT), "a behavioral pattern marked by sluggishness, daydreaming, and hypoactivity," 
(APA, 2000, p. 93) are also likely to remain undetected during their primary and secondary education (Barkley, 2006; Milich, Balentine, \& Lynam, 2001).

Some college students with ADHD may also represent a subset of individuals with ADHD who experience less adaptive impairment. Shaw-Zirt, Popali-Lehane, Chaplin, \& Bergman (2005) found that students who were first diagnosed with ADHD in college had similar symptom patterns as those found in the general population; however, the associated difficulties were less severe. The researchers hypothesized that the development of adaptive compensatory strategies likely contributed to better outcomes. Heiligenstein (1995) also found that $31 \%$ of the students who were diagnosed with ADHD during the 1993 academic year had presented for their first ADHD evaluation. Further, nearly half of these students evidenced no comorbidity.

Many students with undiagnosed ADHD may experience significant academic problems for the first time upon arriving to college as their previous coping mechanisms may not continue to be as effective for the challenging demands of college life (ShawZirt, Popali-Lehane, Chaplin, \& Bergman (2005). These difficulties include a transition to advanced education, which requires sustained attention and inhibition (Weyandt, 2003). At the postsecondary level, students are also now expected to take responsibility for planning, structuring their time, and securing their medical and academic accommodations that were previously taken care of by others (Ramsay \& Rostain, 2006).

A review of studies reporting the neuropsychological performance of college students to date (Weyandt, Linterman, \& Rice, 1995; Weyandt, Rice, \& Linterman, 1998; Weyandt, Mitzlaff, \& Thomas, 2002; Advocat, Martino, Hill, \& Gouvier, 2007) suggests that a test of continuous performance may be the only reliable measure to aid in 
discriminating students with and without ADHD. This finding appears to suggest that college students with ADHD are less impaired than their counterparts in the general population. This suggestion is based, however, on a minimal 4 studies, 3 of which were conducted by the same author, with limited sample sizes, which significantly diminishes power and generalizabiltiy. An area clearly in need of further exploration is the neuropsychological performance of college students with ADHD. Consistency in the methods of assessing for ADHD would enhance this effort.

\section{Assessment for ADHD in Adults}

Assessment for ADHD involves documenting current and past symptoms, establishing that the symptoms cause impairment, obtaining physical, developmental, and psychiatric histories, and cognitive evaluation (Weiss \& Murray, 2003). ADHD in adults is frequently misdiagnosed (Asherson, 2005). The overlap in symptom expression of ADHD and many other psychiatric or comorbid conditions often leads to this misdiagnosis. As there is no single definitive test for identifying ADHD, researchers suggest employing a battery of measures for assessing ADHD in adults (Schoechlin \& Engel, 2005). A diagnosis of ADHD should be systematically based on the individual's developmental and adult psychiatric history and current mental state (Asherson, 2005). There are many rating scales, both self and other report than can be useful in the diagnostic process. However, rating scales alone are not sufficient to ensure a correct clinical diagnosis. Asherson suggests that ADHD is a clinical and behavioral phenotype best evaluated by a clinical diagnostic interview with supporting evidence from informants. A clinical diagnostic interview by a well trained professional is essential in differentiating ADHD from other comorbid and psychiatric disorders. 
Neuropsychological testing can also contribute to an accurate diagnosis of ADHD as "... neurocognitive dysfunction is an integral component of the constellation of symptoms experienced by adults diagnosed with ADHD” (Woods, Lovejoy, \& Ball, 2002, p. 29). Tests of sustained and divided attention have consistently differentiated between adults with and adults without ADHD. Measures of complex information processing speed, response inhibition, and verbal fluency are also useful in identifying the unique functional impairments experienced by adults with ADHD (Woods et al., 2002).

Heiligenstein and Keeling (1995) found high levels of undiagnosed ADHD among college students presenting to university health services for assessment of mood and learning problems. Given the increasing numbers of, and academic difficulty experienced by college students with ADHD, psychologists working in secondary institutions should be well versed in identifying ADHD, particularly the role of neuropsychological functioning.

Neuropsychological Functioning in Children and Adolescents with ADHD

The relationship between executive functioning and ADHD among children and adolescents has been well researched (Biederman et al., 2004; Fischer, Barkley, Smallish, \& Fletcher, 2005; Hinshaw, Carte, Fan, Jassy, Owens, 2007; Schmitz et al., 2002; Verte, Ooserlaan, Roeyers, \& Sergeant, 2005), with results substantiated by metaanalyses (Pennington \& Ozonoff, 1996; Willcutt et al., 2005). Given the general consensus regarding the role of executive functioning deficits in children and adolescents with ADHD, only primary findings from a subset of these studies will be reviewed in the following paragraphs. 
First, Willcutt et al. (2005) conducted a metaanalysis of 83 studies that provided data on children with $(n=3734)$ and without $(n=2969)$ ADHD. Results revealed significant differences between the ADHD group and the control group on all 13 executive functioning tasks, with medium effect sizes across all comparisons. Significant group differences were obtained most consistently with Stop-Signal Reaction Time (response inhibition), Continuous Performance Test (CPT) omission errors (vigilance), planning (i.e. Tower of Hanoi, Porteus Mazes), and spatial working memory. When analyzed according to subtype, there were few consistent differences between ADHD-C and ADHD-I on any executive functioning measure. Only three studies included individuals with ADHD-H. The results found minimal executive functioning impairment among the ADHD-H subtype, suggesting that deficits in executive functioning may be related to symptoms of inattention rather than hyperactivity or impulsivity. Willcutt et al. (2005) conclude that "Executive dysfunction in domains such as response inhibition, planning, vigilance, and working memory plays an important role in the complex neuropsychology of ADHD. Nonetheless, executive functioning weaknesses are neither necessary nor sufficient to cause all cases of ADHD” (Willcutt et al., 2005, p. 1343).

In a seminal metaanalysis, Pennington and Ozonoff (1996) reviewed 18 studies examining executive functioning among children, adolescents, and young adults, ages 6 to 24, with ADHD. Significant differences between individuals with ADHD and controls were found in 15 of the 18 studies. Participants with ADHD performed significantly worse on 40 of the 60 executive functioning measures used in the studies. Further, ADHD participants did not perform significantly better on any of the 60 measures. The authors conclude that, "In sum, across these studies, ADHD subjects fairly consistently 
exhibit significantly poorer performance on measures of vigilance/perceptual speed, whereas they usually perform normally on a variety of verbal and nonverbal measures" (Pennington \& Ozonoff, 1996, p. 64). The Tower of Hanoi (planning), Matching Familiar Figures Test errors (inhibition), Stroop (interference control), Trails B (set shifting), and measures of motor inhibition (i.e. Go-NoGo, Stopping, Anti-Saccade, Conflict Motor Task) were the most consistently impaired measures.

While deficits in executive functioning are clearly prominent among individuals with ADHD, deficits in executive functioning alone do not cause ADHD, nor do they fully account for the symptom expression of the disorder. Guerts, Verte, Ooserlaan, Roeyers, and Sergeant (2005), for example, compared 16 normally developing boys, 16 boys with ADHD-C and 16 boys with ADHD-I on a battery of neurological measures. No significant differences were found between the ADHD-C and ADHD-I subgroups, but a few differences were noted between children with ADHD and normally developing boys. Significant differences were found between children with ADHD-C and non-ADHD children on Stop-Signal Reaction Time and the circle drawing task, both indicators of poor response inhibition, and a measure indicating less accuracy with cognitive flexibility. The ADHD-I group also appeared to be less sensitive to interference than normally developing boys. Differences were not found on measures of verbal fluency, working memory, planning, and an additional measure of cognitive flexibility. The authors discuss these findings in relation to Barkley's theory, suggesting that the results do not support his theory that children with ADHD-C and ADHD-H exhibit broad deficits across executive functioning domains. This study suffers, however, from small sample sizes and the exclusion of females. 
Studies by Schmitz et al. (2002) and Biederman et al. (2004) further elaborate on the role of executive functioning among children and adolescents with ADHD. Schmitz et al. (2002) compared 30 adolescents with ADHD (10 ADHD-I, 10 ADHD-C, 10 ADHD-H) to 60 healthy controls, aged 12 to 16 years, from state schools in Brazil. Four adolescents from the authors' ADHD clinic were also included to complete the ADHD subgroups. The Wisconsin Card-Sorting Test (WCST) was used to assess "broad executive function," the Stroop Test to assess selective attention, and Digit Span and Word Span to assess attention. ADHD diagnosis was assessed according to DSM-IV criteria. There were no significant differences between adolescents with ADHD-H and healthy controls on any of the neuropsychological measures, a finding that mirrors the metaanalytic results from Willcutt et al. (2005). ADHD-C adolescents performed worse than all of the other groups on total errors of the WCST, worse than control adolescents on conceptual responses on the WCST, and worse than ADHD-H adolescents on Digits Backwards, suggesting deficits in concept formation, set-shifting, and verbal working memory. When compared with control students, both the ADHD-I and the ADHD-C students performed worse on Digits Backwards, a measure of verbal memory. ADHD-I adolescents performed worse than control adolescents on the Stroop Color-Word error score and worse than all of the other groups in the time taken to complete the Stroop Color-Word task. The authors suggest that neuropsychological impairment seems to occur only in the ADHD subtypes with clinically significant attentional problems. Further, individuals with ADHD-C appear to perform worse on a broad measure of executive functioning, where individuals with ADHD-I exhibit poorer selective attention skills. Several limitations preclude the generalizability of these results. One limitation is 
the exclusion of any adolescents who had been treated for ADHD. This may limit a particular subset of those with ADHD, potentially those more severely impaired. Psychiatric comorbidities potentially impacting impairment were not assessed. Sample sizes were also very small. Nonetheless, these results suggest that there may be cognitive differences among ADHD subtypes.

Biederman et al. (2004) examine more specifically the role of executive functioning in children and adolescents independent of ADHD, and ADHD independent of executive functioning. The study sample size was large and included 121 male participants with ADHD, 103 male control participants, 138 female participants with ADHD, and 122 female control participants, ages 6 to 17. Psychiatric, psychosocial, cognitive, and neuropsychological assessments were completed. Though executive functions are generally considered to be comprised of several factors, the authors used the battery of neuropsychological assessments as a measure of a single factor. A factor analyses revealed an eigenvalue of 2.66 for the first factor, and 0.26 for the second factor. The authors suggest that these results support the notion that the battery is measuring a single latent construct. Four groups were defined for comparison: (a) control participants without executive function deficits (EFD) (control - EFD), control participants with EFD (control + EFD), ADHD participants without EFD (ADHD - EFD), and ADHD participants with EFD (ADHD + EFD). Participants were considered as having EFD if they scored 1.5 standard deviations below the mean on at least 2 measures of executive functioning.

Results revealed that EFD were more prominent in participants with ADHD (33\% verses $12 \%$, respectively). This association remained after statistical adjustment for 
gender, age, IQ, LD, and SES. In terms of ADHD symptomology, there were no differences on the number of hyperactive-impulsive or total symptoms between ADHD participants with and without executive functioning deficits. However, ADHD + EFD participants experienced significantly more inattentive symptoms than ADHD - EFD. In regards to academic functioning, ADHD participants with and without executive functioning deficits performed worse than control participants on achievement scores and measures of school functioning. Further, the ADHD + EFD participants demonstrated significantly poorer performance on every academic outcome assessed (repeated grade, extra help, special class, LD) relative to the ADHD - EFD participants. Whereas in control participants, school performance did not differ as a function of executive functioning deficits.

Additional analyses on the academic outcomes with ADHD participants found that ADHD + EFD were over 2 times more likely to repeat a grade, and almost 3 times more likely to have a LD when compared with ADHD - EFD participants. In addition, among participants with ADHD, EFD were associated with a statistically significant average decrease of over 10 points on the IQ score, and 4 points on the each WRAT-R score. Global and interpersonal functioning was more impaired among ADHD participants than control participants, but did not differ as a function of executive functioning deficits.

The results of this study reflect those commonly found in other studies (Fischer, Barkley, Smallish, \& Fletcher, 2005; Hinshaw, Carte, Fan, Jassy, \& Owens, 2007; Pennington \& Ozonoff, 1996; Schmitz et al., 2002; Verte, Ooserlaan, Roeyers, \& Sergeant, 2005; Willcutt et al., 2005). First, participants with ADHD were significantly 
more likely to have deficits in executive functioning relative to control participants. Second, individuals with ADHD and executive functioning deficits experienced significantly more inattentive symptoms than individuals with ADHD without deficits in executive functioning. The finding that inattention appears to be strongly linked to executive functioning deficits was also found in the Willcutt et al. (2005) and Schmitz et al. (2002) studies, and will be demonstrated in the adult literature. Third, among participants with ADHD, deficits in executive functioning increased the risk for grade retention, $\mathrm{LD}$, and lower academic achievement. In contrast, deficits in executive functioning among control participants did not affect functional outcomes. Therefore, individuals with ADHD and comorbid deficits in executive functioning are likely to have significantly more academic difficulty, implicating a greater need for assessment, accommodations, and intervention.

The finding of cognitive differences between ADHD subtypes has not been replicated in all studies (Hinshaw, Carte, Fan, Jassy, \& Owens, 2007; Nigg, Blaskey, Huang-Pollock, \& Rappley, 2002). For example, Nigg et al. (2002) compared executive functioning in 105 children, 46 with ADHD-C, 18 with ADHD-I and 41 controls and found minimal subgroup differences between children according to subgroup. Thus, Nigg et al. (2002) suggest that ADHD-C and ADHD-I are related disorders that share deficits in vigilance and may differ only in severity. The arguments regarding ADHD subtypes will be readdressed in the adult literature.

\section{Neuropsychological Functioning in Adults with ADHD}

A substantial amount of research has addressed the neuropsychological functioning of adults with ADHD. This review will begin with examination of individual 
studies, followed by metaanalytic results, and finally research with college students. Given the preponderance of available articles, individual studies were chosen based on the use of a battery of neuropsychological instruments, the inclusion of a college population, studies with strong methodology, and recency of publication.

Studies Utilizing a Battery of Neuropsychological Tests. Several studies have employed a battery of neuropsychological tests to assess executive functioning among adults with ADHD. Most studies include comparisons with a healthy control group, and a few also provide comparison with clinical control groups. The latter comparisons are particularly important for establishing specificity. That is, identifying that the deficits exhibited are specific to ADHD and not also present in other psychiatric disorders.

Utilizing a battery of executive functioning tasks improves the ability to detect specific differences, and has been suggested by prominent researchers in the field. This also allows for detection of inconsistency, which is a hallmark of ADHD (Barkely, 2006). An area that remains of concern, however, are the psychometric properties of executive functioning tests themselves. Most measures of executive functioning were developed a century ago for the assessment of brain damage. Reliability and validity for these measures are often moderate or unknown (Lezak, 2002; Willcutt, Doyle, Nigg, Faraone, \& Pennington, 2005). Pennington and Ozonoff (1996) discuss how the poor measurement properties of executive functioning tasks may contribute to apparent problems with discriminate validity. Further, many of these measures do not allow for differentiation of performance with fundamental skills necessary for task completion versus performance on actual executive functioning skills. This is particularly important in light of Boonstra et al.'s (2005) meta-analytic finding that non-executive functioning tasks provided nearly 
equal discriminatory results for adults with and without ADHD as did executive functioning tasks.

Recently, significant improvements have been made in tests of executive functioning, including updated versions of the tests, with solid reliability, validity and normative data. The D-KEFS, for example, is a battery of executive functioning measures that were standardized based on national norms, and that provide for differentiation of fundamental versus executive functioning skills. Given the recency of its release, and perhaps resistance to change, only a few studies using the D-KEFS are available. Although improvements in executive functioning measures were essential, the accuracy of comparisons of data obtained with different versions could be questionable. Nonetheless, results will be presented based on the data available. Summaries regarding the primary findings regarding executive functioning, the role of comorbidity, and differences between ADHD subtypes will be provided.

Murphy, Barkley, \& Bush (2001) compared the performance of young adults, ages 17 to 28 years, with $(n=105)$ and without $(n=64)$ ADHD on a battery of 14 executive functioning measures and a measure of olfactory identification. Fifty-five percent of the ADHD participants were diagnosed as ADHD-C, 34\% as ADHD-I, $2 \%$ as ADHD-H, and 9\% as ADHD-NOS. ADHD diagnosis was assessed according to DSM-IV criteria with an age of onset criterion adjustment of 12 years of age. The ADHD and control groups did not differ significantly in their age, sex composition, socioeconomic status, or percentage completing high-school. However, the group with ADHD had lower IQ scores and fewer years of education. Further, the young adults with ADHD reported 
significantly more symptoms of depression and oppositional defiant disorder, but not conduct disorder.

The authors present the results as representative of five major domains of executive functioning including: (a) response inhibition, (b) interference control, (c) sustained attention/vigilance, (d) fluency (verbal and ideational), and (e) working memory (verbal and nonverbal). The tests used and their respective executive functioning domain were as follows: Connors CPT (sustained attention and response inhibition); Stroop Color and Word Test (interference); WAIS-III Digit Span Subtest (verbal working memory); WAIS Digit Symbol subtest (attention); Simon game (non-verbal working memory); Controlled Oral Word Association Test (verbal fluency); Object usage test (creativity and ideational fluency); and, the Smell Identification test (olfaction). The authors combined the ADHD-C and ADHD-H subtypes as they shared a deficiency in response inhibition. Individuals with ADHD-NOS were not included in the analyses. An ANOVA revealed no significant differences in the neuropsychological performance between the ADHD-C/ADHD-H and the ADHD-I subgroups. Therefore, to maximize the sample size, all individuals with ADHD were combined into a single group for all subsequent comparisons. ANOVA analyses revealed significant group differences for 11 of the 14 measures in the domains of: inattention (CPT omission errors, CPT reaction time variability, WAIS-III Digit Symbol), response inhibition (CPT commission errors), interference control (Stroop interference, Stroop number completed), verbal fluency (Controlled Oral Word Association Test verbal fluency task), verbal working memory (WAIS-III Digit Span), non-verbal working memory (Simon game), and olfactory 
identification (Smell Identification Test). The three measures that did not differ between the groups were CPT hit reaction time, Stroop number of errors, and the object usage test.

When the analyses were repeated with IQ serving as a covariate, CPT omission errors, WAIS-III Digit Span, and the Controlled Oral Word Association Test did not retain their significance levels. The domains that remained significant include interference control, response inhibition, nonverbal working memory, and 2 of the 3 measures of inattention. Evaluation of sex differences revealed minimal differences. Young women did perform better than young men on the WAIS-III Digit Symbol Subtest and number of errors on the Smell Identification test. Analyses for the impact of comorbidity revealed no differences when covarying for Oppositional Defiant Disorder, and only one significant difference with Major Depressive Disorder on the WAIS-III Digit Symbol Test. Young adults with ADHD and Major Depressive Disorder performed more poorly than young adults with ADHD without Major Depressive Disorder. The relative lack of significant differences found suggested that comorbid Oppositional Defiant Disorder and Major Depressive Disorder are unlikely to account for the numerous deficits in executive functioning found in the study. Future research will need to address the potential impact of other frequently occurring comorbid disorders.

Using a much smaller sample size, and including executive functioning measures omitted in the Murphy et al. (2001) study, Murphy (2002) compared the neuropsychological performance of 18 males with ADHD to 18 control males on two measures of executive functioning, the Tower of Hanoi and the Trail Making Test (TMT) - B, and two control task measures, the TMT-A, and the Benton Facial Recognition Test. The ADHD and control males were recruited from a hospital community and did not 
differ in age or an estimated measure of IQ. A significant difference was found with the non-ADHD males performing significantly better on the Vocabulary subtest of the WAIS. ADHD diagnosis was determined based on a semi-structured interview and comorbidities were ruled out based on the Structured Clinical Interview for DSM-IV Axis I Disorders. Males were included if they met the diagnostic criteria for ADHD and were free of psychosis, major depression, mania, current alcohol and drug abuse, and dependence. All ADHD males met DSM-IV criteria for ADHD-C as children. A kappa value of .83 was obtained from specialist review of a small proportion of videotaped interviews. Results revealed that the ADHD males required a significantly larger number of moves for the Tower of Hanoi, and took significantly longer to complete both the TMT-B and the TMT-A. Males did not differ on time to first move, number of errors or time to solution on the Tower of Hanoi or on the Benton Facial Recognition Test. These results suggest that adult males with ADHD were less efficient at solving the Tower of Hanoi and demonstrated slower visual scanning with both the TMT-A and TMT-B. Murphy (2002) concludes that the deficits found in this study, the ability to complete tasks efficiently, reflect a hallmark of ADHD. This study suggests that although adult males with ADHD exhibit executive functioning deficits, the deficits exhibited are not confined to the executive control domain, a finding that is replicated throughout this review. Limitations to the study include a small sample size and the exclusion of female participants.

Also using a large sample, Nigg et al. (2005) examined the impact of selective executive functioning effects on ADHD symptoms domains among a nonreferred community sample of 195 adults, ages 18 to 37 . ADHD diagnoses were carefully derived 
from multiple self and informant reports and a structured clinical interview, and confirmed by consensus. A battery of neuropsychological tests were used to measure executive functioning, including: Trail Making Test, Stroop Color-Word Test, Wisconsin Card Sorting Test, Logan Stop Task, Tower of London, and Full Scale IQ from the WAIS-III. Significant group differences were found for Trails B Residual, Stop Reaction Time, and Reaction Time variability, measures of cognitive flexibility, inhibitory control, and consistency of sustained attention, respectfully. Stroop word and WCST categories were also significant, but lost significance when IQ was covaried. Covarying for past Major Depressive Disorder, current anxiety disorder, lifetime history of alcohol or substance dependence, and symptoms of antisocial personality disorder resulted in no significant changes in the results.

Further analyses revealed that executive functioning weaknesses observed in the ADHD group were not carried solely by individuals with ADHD-C, considered by some as the most severe type of ADHD. Multinomial logistic regression revealed that membership in the ADHD-C group was predicted by poor executive functioning (Trails B-residual, Stop Task Reaction Time, Stop Task response variability, WCST perseverative errors, WCST categories composite, and Tower total points), but not by slow response speed (Trails A, Stroop word, and Stroop color naming). Whereas, membership in the other ADHD group was predicted by slow response speed, but not by poor executive functioning. The authors discussed these findings as supporting recent two-factor theories of ADHD.

In a related study, Stavro, Ettenhofer, and Nigg (2007), using the same sample as Nigg et al. (2005), examined the relationship between ADHD symptoms, executive 
functioning, and adaptive functioning. Adaptive functioning was measured with the Young Adult Self-Report, Global Assessment of Functioning, and a semi-structured clinical interview. Using Structural Equation Modeling, the authors found a strong correlation between inattentive-disorganized and hyperactive-impulsive symptoms of ADHD, both of which were correlated with weaker executive functioning. Both ADHD symptom domains predicted poorer adaptive functioning, but executive functioning did not predict impairment independently of ADHD, a finding consistent with that found by Biederman et al. (2004). Further, inattentive-disorganized symptoms accounted for $67 \%$ of the variance, compared to only $4 \%$ by the hyperactive-impulsive symptoms. The consistency of these results was confirmed after covarying years of education, IQ and gender. The authors suggest that including a measure of working memory in the executive functioning battery may have provided more meaningful results as working memory has consistently been impaired in adults with ADHD. Nonetheless, "These results suggest that inattentive-disorganized symptoms have lasting and long-term effects upon adaptive functioning in adults with ADHD and may be the primary pathway through which executive functioning is related to adaptive functioning in adults with ADHD” (Stavro, 2007, p. 331).

Most studies comparing ADHD subtypes make those comparisons between individuals with ADHD-C and ADHD-I. Gansler et al. (1998), however, examined cognitive differences among adults with ADHD-I and ADHD-H. When compared with normal controls, adults with ADHD performed worse on three of five measures of executive functioning: TMT, a measure of cognitive flexibility, CPT, a measure of sustained attention, and Auditory Consonant Trigrams (ACT), a measure of short term 
memory. The groups performed comparably on general intellectual functioning (WAIS Block Design and Similarities), and learning and memory (WMS-R Logical Memory and Visual Reproduction). Further, as predicted, ADHD-I performed worse on a measure of short term memory (ACT), where ADHD-H performed worse on a measure of working memory, specifically spatial/temporal organization (WCST). The authors discuss the results as supporting theoretical models in which there are believed to be separate frontal subsystems among ADHD subtypes. Gansler et al. (1998, p. 780) conclude that “... these results may indicate the ADHD subtypes stem from dysregulation of different frontal subsystems and may ultimately be found to respond to different pharmacological and psychological therapies."

Walker, Shores, Troller, Lee, \& Sachdev (2000) compared the neuropsychological functioning of adults with $\operatorname{ADHD}(n=30)$ to a healthy control group $(n=30)$ and a psychiatric group $(n=30)$. Participants completed a neuropsychological battery including measures of intelligence (National Adult Reading Test, Revised), attention (CPT, Digit Span), psychomotor speed (Digit Symbol, Trail Making Test), arithmetic, and executive function (Controlled Oral Word Association Test (COWAT), animal fluency, Stroop). The Wender Utah Rating Scale, Beck Depression Inventory, and State-Trait Anxiety Inventory were used to evaluate ADHD symptoms and psychiatric status. The participants did not differ on gender, years of education, and estimated FSIQ. The psychiatric group was significantly older than both the ADHD and healthy control groups. Comparisons between the ADHD group and the psychiatric group revealed no significant differences. Comparisons between the ADHD group and healthy controls, however, revealed several differences. The ADHD group performed more poorly on the 
CPT omission, CPT commission, CPT hit standard error reaction time, CPT overall index, Digit Symbol, Stroop words, Stroop colors, Stroop interference task, Digits Backwards, and the Controlled Oral Word Association Test, suggesting deficits in sustained attention, response inhibition, psychomotor performance and mental speed, working memory, and verbal fluency. Overall, significant differences were found on 11 of the 18 neuropsychological variables measured. Specificity and sensitivity were also assessed in this study and will be reviewed below. In a relatively small sample, McLean et al. (2004) compared 19 adults with ADHD to 19 matched non-clinical control adults. Adults with ADHD performed significantly worse on spatial working memory, planning, and attentional-set shifting tests, and were significantly slower to respond on the go/nogo task.

While most studies utilized a battery of executive functioning tests encompassing broad domains of executive functioning, a couple of studies assessed for specific domains of executive functioning. Schweitzer, Hanford, and Medoff (2006) assessed the executive functioning domain of working memory, and Epstein, Johnson, Varia, and Conners (2001) assessed the domain of response inhibition. The study by Schweitzer, Hanford, and Medoff (2006) compared a sample of 51 participants (17 ADHD-C, 16 ADHD-I, and 18 normal controls) to assess for differences in working memory performance using the Wechsler Memory Scale $-3^{\text {rd }}$ Edition (WMS-III) and the Paced Auditory Serial Addition Task (PASAT). The groups did not differ by age, education, or IQ. ADHD was assessed using DSM-IV criteria and participants were included based on strict inclusion criteria. Normal controls performed significantly better than both ADHD groups on WMS-III Letter-Number Sequencing, better than ADHD-I on WMS-III Digit Span, and better than 
ADHD-C on both PASAT omission errors and longest sequence. No significant differences in working memory were found between the ADHD-C and ADHD-I subtypes. The authors hypothesize that the finding that the LNS produced the strongest differences suggests that the processing load for adults with ADHD may be taxed as the number of executive functions required to complete a task is increased. The authors also suggest future research including the Sluggish Cognitive Tempo (SCT) type of ADHD-I, as this is believed to be a "more pure form of the ADHD-I subtype" (Schweitzer, Hanford, \& Medoff, 2006, p. 51). The inclusion of this group may increase sensitivity to subtype differences. Limitations to this study include small sample sizes with relatively high IQs.

Epstein, Johnson, Varia, and Conners (2001) compared the performance of adults diagnosed with ADHD to a normal group and a psychiatric control group using three measures of response inhibition, the Continuous Performance Test (CPT), the Posner Visual Orienting Task (PVOT), and the Stop-Signal Task (SST). The participants consisted of 30 normal controls, 25 adults with ADHD, and 15 individuals with an anxiety disorder. Though comorbid diagnoses are presented in table format, the authors did not present statistics related to these potential confounds. The participants completed the Connors Adult ADHD Rating Scale; Speilberger State - Trait Anxiety Inventory; Schedule for the Assessment of Conduct, Hyperactivity, Anxiety, Mood and Psychoactive Substances; and the Semi-structured clinical interview for DSM-IV to determine eligibility for the study. Results revealed that the ADHD group had a significantly higher rate of commission errors than both the anxiety and normal groups on the CPT, which suggests higher rates of impulsive responding. No differences were 
detected between the anxiety and normal control groups. On the PVOT, though not statistically significant, there was a trend for the ADHD group to have increased numbers of impulsive errors compared to the normal group. There were no statistically significant differences between the groups on the SST task, suggesting no differences in their speed in inhibiting a response. In summary, this study found that response inhibition deficits were limited to only one of the neuropsychological tests, the CPT, and these deficits are specific to ADHD as they were not found to be present in the anxiety group. Although some variation was noted, it appears that the results found across the majority of studies using a battery of neuropsychological measures were mostly consistent. Adults with ADHD tend to exhibit broad neuropsychological deficits in the domains of sustained attention, response inhibition, interference control, verbal fluency, verbal and non-verbal working memory, cognitive flexibility, and planning.

Predictive Models of ADHD. While most studies examined differences between adults with ADHD and non-ADHD adults, a few studies sought to establish neuropsychological models predictive of ADHD membership. In addition to the general executive functioning results presented above, Walker, Shores, Troller, Lee, \& Sachdev (2000) also assessed specificity and sensitivity with logistic regression analyses. A model with four predictors (CPT commission errors, CPT reaction time variability, Stroop color-word score, and Digit Symbol) correctly classified 93\% of the ADHD group and $90 \%$ of the healthy control group. Classification for the psychiatric group, however, was poor.

Kovner et al. (1998) examined whether specific neuropsychological testing could differentiate 19 adults with ADHD from 10 individuals with other disorders. Their 
neuropsychological battery included tests in six areas using subtests from the following measures: WAIS-R, Boston Naming Test, Benton Test of Facial Recognition, Wide Range Achievement Test, Gates-McGinite Reading Test, Warrington Recognition Memory Test, Gold Letter-Number-Span Test, Connors Continuous Performance Test and Shifting Sets Test. Participants also completed the Beck Depression Inventory and the Spielberger State - Trait Anxiety Scales. The groups did not differ significantly on major demographic variables, variables associated with learning disabilities, or psychiatric factors. Only three variables differed significantly between the groups: Digits Backwards (WAIS-R), and mean reaction time and reaction time variability from the fourth set of the Shifting Sets Test. Logistic regression revealed that the former two variables were sufficient to discriminate individuals with ADHD from individuals in the control group. Adding the reaction time variability did not improve the predictive power. The authors report that the probability of correctly classifying a randomly selected pair of individuals, one with and one without ADHD, is between 91\% and 95\%. They conclude that adults with ADHD may have deficits in reversing, inhibiting, and re-engaging specific cognitive and motor sets, while deficits were not detected on other cognitive measures of attention (short term memory and working memory) or on other measures of sustained attention (CPTs). Although interesting, these results are based on a very small sample size. Additional studies with sufficient samples sizes are needed to corroborate these results.

In summary, Walker, Shores, Troller, Lee, Sachdev (2000) found that a model with four predictors (CPT commission errors, CPT reaction time variability, Stroop color-word score, and Digit Symbol) correctly classified 93\% of the ADHD group and 
$90 \%$ of the healthy control group. Kovner et al. (1998) also achieved strong predictive power, $91 \%$ to $95 \%$, with only 2 measures, Digits Backwards from the WAIS-R, and mean reaction time from the fourth set of the Shifting Sets Test. While Walker et al. (2000) found the CPT to successfully predict ADHD membership, Kovner et al. (1998) did not.

Alternative Data Analyses and Interpretation. Although few studies have used alternative means of data analyses and interpretation to examine the neuropsychological performance of adults with ADHD, three exceptions are presented. The first, a study by Armstrong, Hayes, and Martin (2001) sought to subtype adults with ADHD based on neuropsychological profiles. Participants consisted of twenty-five adults (16 female, 9 male) who met the DSM-IV criteria for ADHD without comorbidity. Each participant was administered a 5 hour battery of tests of neurocognitive and psychoeducational functioning. Cluster analysis resulted in three subgroups that did not differ in age, ADHD subtype, substance abuse history, smoking history, or previous psychiatric history. The three groups were significantly differentiated by 10 test scores, which were entered into a discriminate analysis. Five of these tests, visual immediate memory span, delayed simple figure recall, immediate and delayed complex figure recall, and object naming, differentiated the three subgroups with an overall accuracy of $96 \%$. The first and second subgroups were differentiated by $100 \%$, and the third subgroup by $86 \%$. The first subgroup was characterized by no neuropsychological impairment, with the exception of impaired CPT scores. Subgroup 2 had selective deficits in speed of visuospatial perception, in working memory for visual information, and in memory for complex information. The third subgroup demonstrated slightly greater cognitive impairment than 
the first two subgroups, with impairment in generalized processing speed, attentional dyscontrol, impairment in most aspects of memory, and in problem solving. The three subgroups were similarly impaired on the CPT. The resulting three subtype classification led to a very high classification of the neurocognitive pattern of individuals with ADHD. The authors discuss the possibility that the third subgroup may represent individuals with learning difficulties that were not severe enough to result in labeling as learning disabled. The results of this study suggest that adults with ADHD exhibit one of three neurocognitive profiles. These neurocognitive profiles do not appear to correlate with the current subsets according to the DSM-IV-TR. Future studies with sufficient sample sizes are needed to determine if these results are replicated.

Woods, Lovejoy, Stutts, Ball, and Fals-Stewart (2002), employed an intraindividual discrepancy analysis to identify patterns of neuropsychological strengths and weaknesses relative to one's global ability level. Significant group differences were identified between the adults with ADHD and controls on each of the neuropsychological measures used in the study. ADHD adults exhibited greater discrepancies between their IQ and measures of cognitive flexibility (SNST), verbal fluency (Controlled Word Association Test, information processing speed (TMT A), divided attention (TMT B), freedom from distractibility (WAIS-R), and recall component of a word list learning (California Verbal Learning Test). Though few studies have used alternative means of data analyses and interpretation, the results of this study show that this may be a next logical step.

A third example of alternate data analyses and interpretation comes from the work of Walker, Shores, Troller, Lee, \& Sachdev (2000). The authors discussed that, when 
compared to normative data (scores at least one standard deviation below the mean) the ADHD group displayed impairment on only 4 measures: CPT inattention, CPT increased response latency with slower stimulus presentation, and slowed information processing on Trails B and Stroop tasks. "These findings suggest that inattention and slowed information processing may be the primary residual neuropsychological impairments in adulthood" (Walker et al., 2000, p. 123).

The results from these studies utilizing alternative methods of data analyses and interpretation are promising. Alternative methods may clarify discrepancies found in traditional studies. Remaining open to unexplored possibilities has been suggested by several researchers. In their concluding thoughts regarding a special edition devoted to adult ADHD, Wolf and Wasserstein (2001) suggest maintaining a broad outlook to avoid premature conclusions and oversimplification of concepts. The authors conclude that " $\ldots$ executive functioning is a central concept in understanding the real world difficulties encountered by adults with ADHD and may underlie much of the disabling aspect of the disorder" (Wolf \& Wasserstein, 2001, p. 404). They caution, however, that the meaning of executive functioning is extremely ambiguous. The results discussed above as well as the conclusions that will be drawn from the metaanalyses mirror the conclusions proposed by Wolf and Wasserstein.

ADHD Subtypes. Several researchers have suggested that inconsistent neurological profiles among individuals with ADHD may be due to deficits specific to ADHD subtype. Although some differences have been noted, the results have been largely discrepant, with a majority suggesting that there are no consistent neuropsychological profile differences among ADHD subtypes. Milich et al. (2001) 
argue that the differences among individuals with ADHD may not be captured according to the current diagnostic subsets. For example, a difference of one symptom (i.e. 6 symptoms of inattention plus 5 hyperactive-impulsive symptoms $=$ ADHD-I; 6 symptoms of inattention plus 6 hyperactive-impulsive symptoms = ADHD-C; 5 symptoms of inattention plus 5 hyperactive-impulsive symptoms $=$ ADHD-NOS) would differentiate individuals who otherwise exhibit a similar symptom presentation. Similarly, individuals with different symptom presentations may receive the same diagnosis (i.e. 9 symptoms of inattention and 0 symptoms of hyperactivity or impulsivity = ADHD:I; 6 symptoms of inattention and 5 symptoms of hyperactivity or impulsivity = ADHD:I). These examples highlight the possibility of obscuring real differences among individuals with ADHD given the current diagnostic subtyping system. Further, as symptoms of hyperactivity and impulsivity tend to diminish over time, children originally diagnosed with ADHD-C may receive a diagnosis of ADHD-I as an adult. A question that remains to be answered is whether there are any clinically relevant differences between the subtypes. It is plausible that there are greater within group differences than between group differences for the ADHD subtypes.

Differences and similarities among individuals with various ADHD symptoms are clearly in need of additional research. This need is particularly prominent in light of hypotheses suggesting that individuals with "sluggish cognitive tempo" (SCT) represent a distinct and unrelated disorder (Barkley, 2006; Milich, Balentine, \& Lynam, 2001). Of particular interest is that items related to SCT were found to specifically correlate with ADHD-I in the DSM-IV field trials (Frick et al., 1994). However, because of their poor negative predictive power, and the DSM-IV workgroup's decision to include a single list 
of inattentive symptoms, the items were excluded from the DSM-IV final diagnostic criteria (Carlson \& Mann, 2002). The debate regarding SCT continues with several researchers suggesting further examination of the role of SCT (Carlson \& Mann, 2002; McBurnett, Pfiffner, \& Frick, 2001; Milich et al., 2001) while others do not (Todd, Rasmussen, Wood, Levy, \& Hay, 2004). According to the DSM-IV-TR, individuals exhibiting the characteristics associated with SCT, namely "a behavioral pattern marked by sluggishness, daydreaming, and hypoactivity" (APA, 2000, p. 93) should be diagnosed with ADHD-NOS. Research addressing individuals with ADHD-NOS may be promising; however, individuals with subthreshold symptoms are also given this diagnosis, again likely obscuring a homogenous presentation.

To further support their hypothesis regarding differences among individuals with various subtypes of ADHD, Milich et al. (2001) provide an excellent review of research addressing ADHD subtypes. They suggest that individuals with ADHD-I are more likely to be shy, passive and withdrawn, less responsive to stimulant medication with effective responses exhibited at a lower dose than the ADHD-C group, and are more likely to have a math disability, suggesting that there is a difference in the core processing deficits in the two groups. In contrast, children with ADHD-C are more likely to be male, to have comorbid externalizing disorders, to have an earlier age of onset or referral, and to be actively rejected by their peers. Milich et al. suggest that the differences exhibited by ADHD-C and ADHD-I, namely, disinhibited, overactive, externalizing, and energetic, verses inhibited, hypoactive, internalizing, and sluggish, respectively, appear to fall at opposite ends of a continuum. Other disorders, such as Conduct Disorder and Oppositional Defiant Disorder are suggested to have more in common with ADHD-C 
than ADHD-I (Milich et al., 2001). Barkley (2006) also emphasizes that the identification of more homogenous, clinically meaningful subtypes of ADHD is immensely important to enable better understanding of the comorbidities, developmental course, etiologies, outcomes, and response to interventions among individuals with ADHD (Barkley, 2006). Results regarding differences in the neuropsychological performance among ADHD subtypes are mixed. Several studies found significant differences between ADHD subtypes, while others did not. Nigg et al. (2005) found that membership in the ADHD-C group was predicted by poor executive functioning, but not by slow response speed. Membership in the other ADHD group was predicted by slow response speed, but not by poor executive functioning. Using the same sample, Stavro, Ettenhofer, and Nigg (2007) suggest that inattentive-disorganized symptoms in adults with ADHD may be the principal pathway through which executive functioning is related to adaptive functioning. Gansler et al. (1998) also found differences in the neuropsychological performance between adults with ADHD-I and ADHD-H, leading the authors to suggest that ADHD subtypes result from dysregulation of different frontal subsystems. However, several studies did not find significant differences in the neuropsychological performance between the ADHD subtypes (Murphy, Barkley, \& Bush, 2001; Schweitzer, Hanford, \& Medoff, 2006). The latter authors also suggest future research including the Sluggish Cognitive Tempo (SCT) type of ADHD-I. The inclusion of this group may increase sensitivity in better understanding subtype differences.

Comorbidity and Specificity. The frequency of comorbid disorders among individuals with ADHD necessitates research evaluating the potential influence of comorbidity on the neuropsychological performance of adults with ADHD. Though it 
might be expected that individuals with ADHD and comorbid disorders would exhibit greater deficits than individuals with ADHD alone, the results are inconsistent. Some studies have found minimal (Murphy, Barkley, \& Bush, 2001) or no differences (Nigg et al., 2005) when controlling for comorbid conditions. The results of a recent metaanalysis, however, revealed that adults with ADHD and a comorbid condition performed worse on most neuropsychological tests (Hervey, Epstein, \& Curry, 2004). Thus, the question of whether comorbid conditions exacerbate neuropsychological deficits among adults with ADHD remains inconclusive.

Studies comparing adults with ADHD to clinical control groups have also revealed minimal differences between the groups (Walker, Shores, Troller, Lee, \& Sachdev, 2000). One exception to this was found by Epstein, Johnson, Varia, and Conners (2001). They found that response inhibition deficits were specific to ADHD when compared with an anxiety clinical control group. Thus, although comorbidity does not appear to negatively impact the neuropsychological performance of adults with ADHD, the deficits exhibited may not be specific to ADHD.

Metaanalytic Studies. The results from metaanalyses will be presented to provide a more robust overview of the research addressing neuropsychological functioning among adults with ADHD. As will become apparent, adults with ADHD largely exhibit deficits in executive functioning, though the particular tasks identified and the executive functions measured by the tasks have differed. Four metaanalyses, three pertaining to executive functioning, one pertaining to IQ, and a meta-analytic review will be presented. In reviewing these metaanalyses, the reader is encouraged to remain aware that many of the studies included in each metaanalysis are also represented by the other metaanalyses. 
Each metaanalysis is unique, however, in their inclusion/exclusion criteria, resulting sample size, and method of data presentation and interpretation.

Boonstra, Oosterlaan, Sergeant, and Buitelaar (2005) conducted a metaanalysis inclusive of 13 studies examining executive functioning and non-executive functioning among adults with and without ADHD. This metaanalysis employed stricter criteria than the other metaanalyses reviewed. First, each study had to include at least one executive functioning measure in one or more of the five domains indicated. Second, studies had to compare the performance of an adult ADHD group with a group of normal control participants, all ages 18 or older. Third, sufficient information for the calculation of effect sizes had to be available. Fourth, diagnoses had to be made according to either DSM-IIIR or DSM-IV criteria. Fifth, executive functioning measures were only included if at least four studies with an adult ADHD sample provided information on the same version of the test and on the same dependent variables. The final criteria required the total number of participants in both the ADHD and control groups to exceed 50 for each dependent variable, in order to obtain sufficient power to find significant results for at least medium effect sizes.

Among the executive functioning measures, medium effect sizes were found with the Controlled Oral Word Association Test (COWAT), CPT attentiveness, CPT commission errors, and the Trail Making Test - B (TMT-B). These results suggest that when compared with non-ADHD adults, adults with ADHD exhibited poorer performance on measures of verbal fluency, the ability to distinguish important from nonimportant information on a stimulus level, response inhibition, and set shifting. A small effect size was also found for WAIS Digit Span Backward, indicating that adults with 
ADHD exhibited more difficulty with verbal working memory than did the control group. The groups did not differ in their response style to the CPT as indicated by the CPT risk taking score. In regards to non- executive functioning results, medium effect sizes were found for Stroop Color Card, Stroop Word Card, CPT omission errors, and CPT variability in reaction-times, suggesting difficulty with color name reading, color naming, and vigilance. Small effect sizes were also found for the TMT-A and the WAIS Digit Span Forward, suggesting slight deficits in serial information processing, visual scanning, motor speed, and verbal memory span.

Boonstra et al. (2005) discuss their findings in light of theories of ADHD. They suggest that poor performance on tests such as the TMT-A, WAIS Digit Span Forward, Stroop, and CPT omissions, seem to point towards general slowing on more cognitive responses, even though motor response (CPT reaction time) is not slower. Boonstra et al. question the validity of theories of ADHD based largely on executive functioning. Averaging the effect sizes across the executive functioning domain and the non-executive functioning domains, resulted in similar effect sizes (.40, and .43 , respectively). The authors suggest that individuals with ADHD exhibit deficits in various areas of cognitive functioning, including executive functioning. They also emphasize the importance of differentiating between fundamental cognitive function and executive functioning.

Schoechlin and Engel (2005) conducted a meta-analysis to review the relationship between subjective reports and objective neurological tests of cognitive deficits. Twentyfour studies using one of 50 common neuropsychological tests to examine the neurocognitive performance of clinically diagnosed ADHD patients aged 16 years or older to a control group were included. Of the thirty-seven studies identified, thirteen 
studies were excluded due to use of uncommon neuropsychological tests, incompatible experimental design, or limit data availability. Unlike the Boonstra metaanalysis, six of the studies in the current metaanalysis included a clinical group, either serving as the control group, or were presented in addition to a normal control group. A total of 867 ADHD adults and 806 controls were included. The patients had a mean age of 31 and most had high levels of education. Eighteen studies compared ADHD patients to healthy controls, six used an outpatient control group, and 3 studies included ADHD patients with a comorbid major psychiatric disorder (depression, substance abuse, obsessive compulsive disorder). Psychotropic drugs were not permitted prior to testing in most of the studies.

Schoechlin and Engel (2005) assigned each measure to 1 of 10 functional domains, though there may have been more than one fit for the measures. The domains and corresponding measures of executive functioning are as follows: a) Verbal intelligence (WAIS-R Similarities, Information, Vocabulary, Reading); b) Executive functions (Wisconsin Card Sorting Test (WCST); Tower of Hanoi); c) Fluency (Controlled Oral Word Association Test; Design Fluency); d) Visual-figural problem solving (WAIS-R Block Design, Picture Completion, Picture Arrangement; ReyOsterrieth Complex Figure; Embedded Figures Test); e) Abstract problem solving/working memory (WAIS-R arithmetic; Auditory Consonant Trigrams); f) Simple attention (Digit Span Forwards; Freedom-from-Distractibility (WAIS-R); Stroop Word Color; Trail Making Test-A; Visual CPT reaction time; 3RT Simple; Wechsler Memory Scale-Attention, Target Orientation Test); g) sustained attention (different versions of the CPT); h) Focused attention (WAIS-R Digit Symbol; Digit Span Backwards; Stroop 
Interference; Trail Making Test-B; reaction time pattern comparison, GDS distractibility, TOAD); i) Verbal memory (California Verbal Learning Test; Wechsler Memory Scale); and j) Figural memory (Rey-Osterrieth Complex Figure Test; Kimura Recurring Figures Test).

Results revealed that ADHD patients exhibited significant performance deficits in 8 of 10 functional domains. Verbal memory, focused attention, sustained attention, and abstract problem solving requiring working memory were the four domains with the most pronounced deficits for ADHD patients. Surprisingly, small effect sizes were found for the executive functioning domain, which included measures of cognitive flexibility and planning. Though results have been mixed for the Tower Test, the finding that adults with ADHD do not exhibit deficits on the WCST is a finding that has been replicated in many studies. The authors also suggest that the results may be confounded by not differentiating among the diagnostic subgroups of ADHD. They suggest that further research focusing on subtypes among adults may provide more consistent neuropsychological profiles. The results of this metaanalysis suggest that overall, adults with ADHD appear to exhibit deficits in tasks requiring focused and sustained attention, verbal memory, and abstract problem solving requiring working memory.

In the most inclusive metaanalysis, Hervey, Epstein, and Curry (2004) included 33 studies that provided data from neuropsychological testing in adults with ADHD. Inclusion criteria included published studies with participants aged 18 years or older who were diagnosed with ADHD as well as a control group. While Schoechlin and Engel (2005) established 10 cognitive domains, Hervey et al. (2004) present results within seven domains: a) attention, b) response inhibition, c) other tests of executive 
functioning, d) memory, e) processing speed and motor speed, f) intelligence, and g) a category labeled "other." Hervey et al. also include effect sizes calculated for single studies. Although these are meaningful, less emphasis will be placed on them.

In regards to the attention domain, medium effect sizes were found for measures of omission and variability in reaction time with CPTs, and the Stroop Color-Word test. Large effect sizes were found for the total time with the Paced Auditory Serial-Addition Task-Revised, and reaction time variability with the Shifting Sets Task, although these results are based on only one to two studies. These results, using standardized and commonly accepted measures of attention, suggest that adults with ADHD exhibit deficits in attention when compared with controls. Response inhibition, as measured by CPT commission errors and Reaction Time on Stop Signal Task trials, also appears to distinguish adults with ADHD from non-ADHD adults with medium and large effect sizes, respectfully.

Results from other tests of executive functioning are mixed. Medium effect sizes were found for TMT-B and the Controlled Word Association Test, suggesting deficits in set-shifting and verbal fluency. Although reported by only a single study, medium to large effect sizes were also found for the Animal Fluency Test, a measure of verbal fluency, and the Rey-Osterrieth Complex Figure Test and Tower of Hanoi, both of which assess planning abilities. Consistent with other studies (Schoechlin \& Engel, 2005; Woods, Lovejoy, \& Ball, 2002), the WCST, a classic test of executive functioning, demonstrated almost no between groups effect. Thus, executive functioning involving complex motor movements or verbal mediation requiring planning and cognitive 
flexibility are abilities more likely to be impaired in adults with ADHD as compared with non-ADHD adults.

Adults with ADHD exhibited deficits in memory, as identified by medium to large effect sizes on 6 of 7 items from the California Verbal Learning Test. Memory deficits were also detected with the Auditory Consonant trigrams, but this has been substantiated by only one to two studies. Interestingly, adults with ADHD did not appear to have difficulty with memory when presented with figural visual stimuli, as evidenced by tests of Visual Reproduction on the Wechsler Memory Scale and memory measures from the Rey-Osterrieth Complex Figure Test.

Measures of processing and motor speed are particularly important in determining whether reaction time or motor speed account for poorer performance on neuropsychological tests. These meta-analytic results suggest that in general, adults with ADHD perform similarly to non-ADHD adults on processing and motor speed. "One trend that can be seen in these data is that when increasing cognitive processing demands co-occur with motor activity, there appears to be a relative increase in response time as a function of those processing demands" (Hervey, Epstein, \& Curry, 2004, p. 494).

Hervey et al. (2004) conclude that neuropsychological deficits among adults with ADHD are demonstrated by a wide variety of performance differences. The authors note that deficits were demonstrated in a portion of the assessment instruments used within each domain, which does not implicate a domain specific neuropsychological deficit. Further, the presence of deficits in non-executive functioning tasks such as memory, processing speed, and motor speed suggests that deficits in executive functioning only partially characterize the impairments among adults with ADHD. 
Most of the studies in this metaanalysis included a measure of IQ either as a sample descriptor, a baseline measure of cognitive performance, or an outcome measure. Overall, adults with ADHD performed worse than non-ADHD adults on the measures of intelligence. Effect sizes in the medium range were found for the Arithmetic and Digit Symbol subscales, suggesting that they may measure cognitive processes related to ADHD deficits. Measures of creativity did not appear to discriminate adults with ADHD from non-ADHD adults. Overall, the results from the Hervey et al. (2004) metaanalysis suggest that neuropsychological deficits are expressed in adults with ADHD across many domains of functioning, with prominent deficits in attention, memory, response inhibition, planning, set-shifting, and verbal fluency, whereas normal performance is noted in simple reaction time.

A topic of significant debate is the role of IQ in studies of executive functioning among individuals with ADHD (Bridgett \& Walker, 2006). A lower IQ is generally found among individuals with ADHD, though results have not been consistent (Bridgett \& Walker, 2006; Hervey, Epstein, \& Curry, 2004). The debate regards whether IQ accounts for executive functioning deficits, executive functioning deficits account for differences in IQ, or the role of shared variability. Researchers have provided compelling arguments for each of the possibilities. Hervey, Epstein, and Curry (2006, p. 498) argue that "removing variability associated with differential IQ performance may be removing part of what constitutes ADHD.” Bridgett and Walker (2006) also argue that a diagnosis of ADHD does not necessitate that IQ be controlled for. However, given that no consensus has been reached regarding this question, it has been recommended that results 
be presented both with and without controlling for or covarying IQ (Bridgett \& Walker, 2006; Murphy, Barkley, \& Bush, 2001).

Bridgett and Walker (2006) conducted a meta-analysis to highlight intellectual differences between adults with and without ADHD. Thirty-three studies, collectively comparing 1512 adults with ADHD and 1258 non-ADHD adults, were included in the metaanalysis. A significant, but small, mean effect size from analysis of WAIS-derived FSIQ revealed that non-ADHD adults intellectually outperformed adults with ADHD by an average of 2.94 IQ points. These results are moderated, however, by the diagnostic criteria used, method used to reach a diagnosis of ADHD, and inclusion of comorbidity. For example, studies that established the ADHD diagnosis with outside documentation found adults with ADHD to outperform non-ADHD adults. Among studies with strict exclusionary criteria, adults with and without ADHD performed similarly.

Consistent with results for overall estimates of FSIQ, adults with ADHD performed significantly lower than non-ADHD adults on several index and subtest findings. These include: Verbal IQ, Performance IQ, Verbal Comprehension, Working Memory, Freedom from Distractibility, Block Design, Digit Symbol, Digit Span, and Arithmetic. The authors suggest that in isolation, these results are not clinically significant, since they are moderated by several variables. When these variables are taken into account, adults with ADHD may perform similarly, below, or even above nonADHD adults on measures of intellectual functioning. An additional avenue for exploration, as suggested by Woods, Lovejoy, and Ball (2002), is the discrepancy between an individuals scores. Woods et al. (2002) found that adults with ADHD have demonstrated index score discrepancies. Particularly, adults with ADHD have scored 8.3 
points lower on Working Memory Index then Verbal Comprehension Index, and 7.5 points lower on Processing Speed Index than Perceptual Organization Index. Thus, the evaluation of IQ discrepancy scores may provide a valuable addition to a battery of neuropsychological measures recommended for the identification of ADHD among adults.

In summary, the four metaanalyses provide similar conclusions. In the most exclusive metaanalysis (Boonstra et al., 2005), verbal fluency, response inhibition, and set shifting were the three domains of executive functioning that discriminated adults with ADHD from non-ADHD adults. Boonstra et al. also found that the non-executive functioning tasks of color name reading, color naming, and vigilance provided nearly equal discriminatory results. Schoechlin and Engel (2005) found that fluency (verbal and nonverbal), focused and sustained attention, and abstract problem solving requiring working memory successfully discriminated adults with ADHD from non-ADHD adults, but found only a small effect size for executive functioning. In the most inclusive metaanalysis, Hervey et al. (2004) also found that verbal fluency, attention, memory, response inhibition, planning and set shifting distinguished ADHD participants from participants without ADHD. However, the results from Hervey et al. (2004) do not indicate domain specific deficits as impairments were found in a portion of the assessment instruments used within each domain, including non-executive functioning tasks. In addition, Hervey et al. (2004) and Bridgett and Walker (2006) found that adults with ADHD tend to perform worse on measures of IQ. All together, it appears that adults with ADHD exhibit deficits in a portion of instruments used to asses the executive 
functioning and non-executive functioning domains of verbal fluency, attention, working memory, response inhibition, set shifting, and planning.

As expected, these results mirror those found from individual studies. Further, the conclusions drawn were also similar to those reported in a qualitative literature review of 35 studies examining the neuropsychological characteristics of adults with ADHD. Woods, Lovejoy, and Ball (2002) found that adults with ADHD exhibited deficits on sustained attention, response inhibition, verbal fluency, interference control, and working memory when compared to normal controls. Woods et al. (2002) noted, however, that measures of verbal fluency and interference control have not consistently discriminated adults with ADHD from adults with psychiatric disorders. They also found that the Wisconsin Card Sorting Test was consistent in poorly differentiating adults with ADHD from healthy controls.

In light of the review of metaanalytic results, limitations to metaanalyses must be noted. First, individual differences in studies cannot be accounted for in a metaanalysis. For example, the diagnostic procedures for identifying and confirming ADHD differ among the studies (Hervey, Epstein, \& Curry, 2004; Schoechlin \& Engel, 2005). The heterogeneity of ADHD, particularly in regards to subtypes, may "hide" the presence of differences when they are lumped together (Hervey et al., 2004). Given the frequent comorbidity with other psychiatric disorders, which are sometimes controlled for and sometimes not, questions are raised question regarding the specificity of neuropsychological performance to ADHD verses a comorbid disorder (Boonstra, Oosterlaan, Sergeant, \& Buitelaar, 2005; Hervey et al., 2004; Schoechlin \& Engel, 2005). Some studies match participants and control for various factors such as education, 
socioeconomic status, and IQ while other studies do not (Hervey, Epstein, \& Curry, 2004). Publication bias is also a potential problem (Boonstra et al., 2005; Pennington \& Ozonoff, 1996). Calculation of a "Fail Safe N" can account for the potential influence of studies finding non-significant results, but Fail Safe N's are not always calculated (Schoechlin \& Engel, 2005). Further, the metaanalyses presented here include many of the same studies. Seven of the twelve individual studies presented were also included in one or more of the metaanalyses. A review of the metaanalyses are presented, nonetheless, as a measure of the robustness of the results found in individual studies and across the metaanalyses regardless of their exclusion/inclusion criteria and sample sizes. Neuropsychological Functioning in College Students with ADHD

While meta-analytic results are available for neuropsychological functioning in adults with ADHD, only a few studies have examined such functioning in college students. Advocat, Martino, Hill, and Gouvier (2007) compared the Continuous Performance Test performance of 95 college students who presented for a comprehensive psychoeducational evaluation at a large southern university between the spring of 2000 and the spring of 2003. The participants were grouped into four groups, using criteria in the DSM-IV-TR to determine diagnoses: college students with ADHD $(n=26)$, students with no diagnosis $(n=30)$, students with a psychiatric disorder $(n=17)$, or students with various cognitive deficits $(n=22)$. There were no significant differences between the groups in age, gender distribution, race, or the Wechsler Adult Intelligence Scale-Third Edition Full Scale IQ. The average IQ of all groups was in the normal range. Results revealed that although the ADHD group performed worse than the other groups on four of the five measures (omissions, response time, response time standard error, standard 
error variability), parametric analyses revealed no significant differences among the groups.

Weyandt, Mitzlaff, and Thomas (2002) compared a group of individuals, primarily college students, with ADHD $(n=17)$ to a control group consisting entirely of college students $(n=62)$. Participants were administered the Test of Variables of Attention (a CPT test) and the Wechsler Adult Intelligence Scale-Revised. The only significant difference between the ADHD and non-ADHD group was found on the TOVA errors of omission, suggesting a deficit in vigilance. The small sample, particularly for the ADHD group, and an unknown percentage of participants with ADHD who were recruited from the university or the community, limited the generalizability of the study. Further, the diagnosis of ADHD was accepted via simple written documentation by the office of disability services, a psychologist or a physician. Therefore, the accuracy of the diagnoses are unknown.

Weyandt, Rice, and Linterman (1998) compared the neuropsychological performance of college students with ADHD $(n=21)$ to students with developmental reading disorders $(D R D)(n=19)$ and controls $(n=24)$. Results revealed that the DRD group exhibited more errors on the WCST than both the ADHD group and the control group. The ADHD group did not perform differently than either group on any of the additional neurological measures: Towers of Hanoi, and TOVA (CPT). These results must be interpreted with caution given the extensive limitations of the study including small sample sizes, and less stringent inclusion criteria, with 8 of the 21 individuals with ADHD continuing the use of their medication at the time of testing. 
Weyandt, Linterman, and Rice (1995) explored the neuropsychological performance of 35 students (17 with high ADHD symptoms, 18 controls) with and without reported ADHD symptoms. The students completed the Adult Rating Scale (ARS), the Wender Utah Rating Scale (WURS), the Brief Symptom Inventory (BSI), and the following neuropsychological tasks: Wisconsin Card Sorting Test (WCST), Stroop Screening Test, Visual Search Attention Test, and Raven's Coloured Progressive Matrices (RCPM). Students with ratings that were 1.5 standard deviations above the group mean on the ARS and the WURS were considered to have high ADHD symptoms. Students that scored within 0.5 standard deviations of the mean on the ARS and WURS were randomly chosen as the control group. In regards to neuropsychological tasks, there was only one significant difference found, albeit in the reverse direction hypothesized. The group with high ADHD symptoms scored significantly better than the control group on the RCPM. Further, no gender differences were found between males and females on the rating scales or the neuropsychological tasks. The authors suggest that the lack of significant differences was not unexpected given the inclusion criteria, small sample size, and low statistical power. Another possibility could be that college students with ADHD exhibit fewer cognitive deficits than adults with ADHD in the general population. Although the results are intriguing, they should be interpreted with caution given the limitations discussed above and the lack of a confirmed ADHD diagnosis as defined by the DSM-IV-TR.

A paucity of research addressing the neuropsychological performance of college students is apparent. A review of the available studies suggests that impaired performance on a measure of continuous performance (CPT, TOVA) may be the only 
reliable measure to aid in discriminating students with and without ADHD. This suggestion is based on a minimal 4 studies, 3 of which were conducted by the same author, which significantly diminishes generalizabiltiy. An area clearly in need of further exploration is the neuropsychological performance of college students with ADHD. Perhaps college students with ADHD do represent a specific subset of individuals with ADHD who are less impaired, as hypothesized by Heiligenstein, Conyers, Berns, and Miller (1998). The recent changes in ADA, however, may alter the make-up of college student populations. Given this potential change, a review of recommended treatments for ADHD will be provided.

\section{Treatment for ADHD}

Asherson (2005) recommends that treatment for ADHD should be multimodal, including pharmacotherapy, psychoeducation, and psychotherapeutic interventions such as coaching, cognitive behavioral therapy, or other types of counseling. Ramsay and Rostain (2006) emphasize the important contribution that neuropsychological testing can provide in identifying areas of relative strength and weakness that could affect functioning. The authors suggest that the identification of strengths and weakness may be particularly useful for college students seeking academic accommodations. Ramsay and Rostain (2006) recommend the use of a neuropsychological battery inclusive of a measure of intelligence, continuous performance, working memory, and executive functions to provide an overview of the student's strengths and weaknesses. This information can guide the development of treatment plans tailored to each student's relative strengths and weaknesses. Unfortunately, research investigating the use of neuropsychological testing in guiding treatment planning is not currently available. 
However, many of the treatments as recommended by Asherson (2005) are available for college students diagnosed with ADHD, and will be elaborated below.

Medication. Stimulant medication is considered the treatment of choice for ADHD in both children and adults (Asherson, 2005; Faraone, Spencer, Aleardi, Pagano, \& Biederman, 2004; Fitzgerald, 2001; Weiss, Murray, \& Weiss, 2002). Results from a recent systematic literature review of pharmacotherapy for adults with ADHD concluded that compounds with predominantly dopaminergic and/or noradrenergic properties appear to be the most efficacious for treating ADHD (Wilens, 2003). Atomoxetine (Strattera), a norepinephrine reuptake inhibitor, is the first non-stimulant drug approved for the treatment of ADHD (Asherson, 2005). Though less effective than stimulants, atomoxetine causes few adverse side effects, and may be particularly useful for adults with ADHD and comorbid anxiety, mood or tic disorders. Bupropion (Wellbutrin) and tricyclic antidepressants also appear useful for adults who do not respond to stimulants, and for adults with concurrent psychiatric disorders (Fitzgerald, 2001). While stimulants produce an immediate effect, antidepressants typically require up to four weeks. To date, serotonergic antidepressants do not appear useful for reducing ADHD symptoms (Fitzgerald, 2001).

In addition to symptom reduction, children with ADHD have shown improvements in performance on tests of executive function, attention, and vigilance following stimulant medication (Mehta et al., 2004). Adults with ADHD have also demonstrated improvements in response inhibition (Schweitzer et al., 2004), and working memory, sustained attention, and motor and processing speed following methylphenidate (Turner, Blackwell, Dowson, McLean, \& Sahakian, 2005). Further, methylphenidate has 
been associated with improved occupational and marital dysfunction (Wender, Wolf, \& Wasserstein, 2001). Though medication can improve many of the core symptoms of ADHD, it does not inherently provide individuals with coping skills and strategies for managing functional impairment (Safren, Sprich, Chulvick, \& Otto, 2004). Quality of life impairments associated with ADHD (i.e. relationship difficulties, underachievement, guilt) encourage the application of additional psychotherapeutic interventions (Safren et al., 2004).

Psychotherapy. While medication is considered a first-line treatment for ADHD, therapeutic interventions such as psychotherapy, coaching, social skills training, support groups, and group therapy have also demonstrated some efficacy in improving functioning among adults with ADHD (Asherson, 2005; Safren, 2006; Weiss, Murray, \& Weiss, 2002). Weiss et al. (2002) emphasize that treatment of ADHD should include both medication and psychotherapy. Psychotherapeutic treatments can aid in teaching specific strategies to improve daily functioning, in modifying beliefs about the meaning of ADHD symptoms, and in addressing any problems that may have developed as a result of the ADHD.

Psychotherapy for adults with ADHD typically involves cognitive and behavioral interventions (Safren, Sprich, Chulvick, \& Otto, 2004). Behavioral interventions are geared toward helping adults develop compensatory strategies for core neuropsychiatric deficits. Cognitive interventions focus on improving cognitive styles and problem-solving strategies, in effort to reduce avoidance of tasks that place adults at risk for academic, mood and relationship problems. Safren et al. (2004) emphasize the importance of 
compensatory strategies (i.e. step-by-step planning, sequential goal attainment) for managing the attentional, planning, and organizational deficits that characterize ADHD. Safren (2006) and colleagues developed a Cognitive Behavioral Therapy (CBT) approach to ADHD treatment in adulthood, and completed a randomized controlled trial of the treatment. Participants in the study were assigned to receive CBT treatment or to continue with pharmacology alone. Adults who received the CBT treatment exhibited a decrease in ADHD symptoms, as well as a decrease in symptoms of anxiety and depression. The CBT treatment protocol was organized into three core modules (organizing and planning, distractibility, and cognitive restructuring) and three optional modules (procrastination, anger and frustration management, and communication skills). The first module involved providing rationale for the treatment, initiating a calendar and notebook, and training in problem-solving skills and prioritization. Problem-solving skills focused on learning to manage external demands by breaking tasks into manageable steps, which reduces the likelihood of avoidance. The second module focused on identifying the limits of each individual's attention span, learning to divide goal-directed activities into steps that correspond to their attention span limits, and writing down distracting thoughts so they can be attended to later. Module three focused on improving adaptive thinking (Safren, 2006).

Although empirical investigations of psychotherapeutic approaches for ADHD are just beginning, the results from Safren (2006) suggest that psychotherapeutic approaches can significantly add to the benefit achieved by medication. This treatment modality may also be particularly helpful for individuals who do not respond to medication or cannot tolerate the side-effects. To date, research investigating the efficacy of interventions 
tailored specifically to an individual's strengths and weaknesses as identified by neuropsychological testing is not available. Future research in this area may prove particularly useful for college students with ADHD.

Academic Accommodations

Post secondary institutions are required to provide accommodations to qualified students with disabilities. Disability is defined by the ADA and Section 504 as “... a physical or mental impairment that substantially limits one or more major life activities" (ADA, 1990). An individual is considered "substantially limited" if they are "... unable to perform major life activities, or significantly restricted as to the condition, manner, or duration under which a major life activity can be performed, in comparison to the average person" (ADA, 1990). Institutions are required to make modifications to academic requirements as necessary to ensure that students with disabilities are not discriminated against. Accommodations are determined on an individual basis and may include: extended time in which to complete examinations, note taking services, a distraction free environment for examinations, and extended deadlines (Ramsay \& Rostain, 2006; Wolf, 2001). The accommodations are often prescribed by an institution's office of Disability Services, following the provision of thorough documentation of the disability. The Office of Disability Services at this large university in the mid-Atlantic region, for example, requires "appropriate documentation" for an ADHD diagnosis prior to receiving disability based accommodations. The requirements for documentation include an evaluation that has been signed and dated by a qualified or licensed professional, testing that utilized adult normed tests such as the WAIS-III, and comprehensive evaluation of 
aptitude (IQ), achievement (Reading, Math, and Written Language), and processing (speed, visual-motor and auditory).

Research has supported the efficacy of several academic interventions in improving the academic performance of students with ADHD. Raggi and Chronis (2006) provide a review of the available literature for addressing academic impairment in children and adolescents with ADHD. The authors concluded that the following interventions have proven beneficial for children and adolescents with ADHD: classwide peer tutoring and parent tutoring (i.e. one-to-one instruction, immediate and frequent feedback), strategy training (i.e. note taking, study and organizational skills), instructional and task modifications (i.e. assignment choices, oral presentation, explicit instructions, computer-assisted instruction), self-monitoring and reinforcement, homework interventions including goal setting and external structuring, and classroom function assessment procedures. The authors elaborate that peer tutoring, self-monitoring, and multimodal programs which employ several of the above interventions appear to have the largest effect on improving the functional impairment of students with ADHD (Raggi \& Chronis, 2006).

Literature regarding the efficacy of academic accommodations for college students with ADHD is largely absent. Although academic accommodations for college students with ADHD make intuitive sense, most lack empirical studies investigating their effectiveness (Weyandt \& DuPaul, 2006). Additional research with non-medical interventions is clearly needed to guide practice with the growing population of college students with ADHD (Weyandt and DuPaul, 2006). 
Individuals diagnosed with ADHD often experience lifelong impairment in several functional domains. Deficits in executive functioning have been theorized as underlying the symptom appearance of ADHD in adults. Neuropsychological tests examining this hypothesis have suggested that adults with ADHD exhibit broad deficits in executive functioning; however, the results have been inconsistent. In addition, deficits in non- executive functioning tasks, such as visual scanning, simple attention, word reading, and uncomplicated processing speed have also been identified among adults with ADHD. Several authors have recommended that a battery of neuropsychological measures be used to identify executive functioning, rather than relying on a single measure. Neuropsychological tests with improved reliability, validity, and normative data have also been called for (Pennington \& Ozonoff, 1996).

The assessment of ADHD, as conducted at this university includes a comprehensive battery of neuropsychological measures with solid psychometric properties. Assessment instruments used include the: Delis-Kaplan Executive Function System (D-KEFS), Integrated Visual \& Auditory Continuous Performance Test (IVA), Wechsler Adult Intelligence Scale-Third Edition (WAIS-III), College ADHD Response Evaluation - Student Response Inventory (CARE-SRI), and College ADHD Response Evaluation - Parent Response Inventory (CARE-PRI). The D-KEFS provides a battery of tests of executive functioning that have been normed on a nationally representative sample (Delis, Kaplan, \& Kramer, 2001). The D-KEFS also provides additional scores which enable the differentiation of fundamental and executive functioning skills. This information provides for a better understanding of the cognitive skills and deficits exhibited by college students with ADHD. In addition, the IVA, a CPT that includes both 
auditory and visual modalities across periods of frequent and non-frequent responding, provides information on attention and response inhibition (Sandford \& Turner, 2000). The WAIS-III, a nationally normed instrument used to assess general cognitive abilities, provides a measure of working memory, a domain that has been associated with ADHD (Wechsler, 1997). Finally, the CARE is a self-report assessment system designed exclusively for individuals at the university level, and allows for a cross-informant, multidimensional evaluation of behaviors common to ADHD (Glutting, Sheslow, \& Adams, 2002). Additional psychometric properties of the instruments used are presented below.

To date, there are few studies examining the neuropsychological functioning of college students with ADHD. Better understanding the neuropsychological strengths and weaknesses among college students with ADHD may improve treatment planning and enable us to provide these students with the support they need not only for academic success, but also for an improved quality of life. Purpose of the Current Study

The purpose of the current study was to examine neuropsychological functioning among college students with ADHD using a comprehensive battery of neuropsychological measures with solid psychometric properties.

Research Question 1: Do students diagnosed with ADHD exhibit deficits in cognitive functioning as compared to the WAIS-III standardization sample? (Measured by the WAIS-III)

Hypothesis 1: There will be no statistically significant difference in the WAIS-III IQ index scores (Verbal Comprehension Index (VCI), Perceptual Organization 
Index (POI), Working Memory Index (WMI), and Processing Speed Index (PSI)) obtained for the ADHD group when compared with the WAIS-III standardization sample.

Research Question 2: Do students diagnosed with ADHD exhibit deficits in attention and response control as compared to the IVA standardization sample? (Measured by the IVA)

Hypothesis 2: There will be no statistically significant difference in attention and response control scores (Full Scale Attention Quotient, Full Scale Response Control Quotient) obtained for the ADHD group when compared with the IVA standardization sample.

Research Question 3: Do students diagnosed with ADHD exhibit deficits in cognitive functioning as compared to students diagnosed with ADHD and a comorbid LD? (Measured by the WAIS-III)

Hypothesis 3: There will be no statistically significant difference in WAIS-III IQ index scores (VCI, POI, WMI, and PSI) obtained for students diagnosed with ADHD as compared to students diagnosed with ADHD and a comorbid LD. Research Question 4: Do students diagnosed with ADHD exhibit deficits in attention and response control as compared to students diagnosed with ADHD and a comorbid LD? (Measured by the IVA)

Hypothesis 4: There will be no statistically significant difference in IVA attention and response control scores (Full Scale Attention Quotient, Full Scale Response Control Quotient) obtained for students diagnosed with ADHD as compared to students diagnosed with ADHD and a comorbid LD. 
Research Question 5: Do students diagnosed with ADHD exhibit significant and unusual discrepancies between their index scores (VCI/POI, VCI/WMI, VCI/PSI, POI/WMI, POI/PSI, WMI/PSI) as compared to the estimated base rates of such differences occurring in the WAIS-III standardization sample? (Measured by the WAIS-III) Hypothesis 5: Students diagnosed with ADHD will not exhibit significant and unusual discrepancies between their index scores (VCI/POI, VCI/WMI, VCI/PSI, POI/WMI, POI/PSI, WMI/PSI) as compared to the estimated base rates of such differences occurring in the WAIS-III standardization sample.

Research Question 6: Do students diagnosed with ADHD exhibit deficits in executive functioning ( $\geq 1$ SD below standardization sample mean)? (Measured by the D-KEFS) Hypothesis 6a: Students diagnosed with ADHD will not exhibit deficits in cognitive flexibility as measured by the following D-KEFS scores: TMT Number-Letter Switching Completion Time, Verbal Fluency Category Switching Total Correct Responses, Design Fluency Total Correct Switching, and ColorWord Interference Test Inhibition/Switching Completion Time.

Hypothesis 6b: Students diagnosed with ADHD will not exhibit deficits in verbal fluency as measured by the following D-KEFS scores: Verbal Fluency Letter Fluency Total Correct, and Verbal Fluency Category Fluency Total Correct. Hypothesis 6c: Students diagnosed with ADHD will not exhibit deficits in nonverbal fluency, as measured by the following D-KEFS scores: Design Fluency Total Correct Filled Dots, and Design Fluency Total Correct Empty Dots. Hypothesis 6d: Students diagnosed with ADHD will not exhibit deficits in concept formation as measured by the following D-KEFS scores: Sorting Test 
Free Sorting Confirmed Correct Sorts, Sorting Test Free Sorting Description Score, Sorting Test Sort Recognition Description Score, Twenty Questions Test Initial Abstraction Score, Proverb Test Free Inquiry Total Achievement Score, and Word Context Total Consecutively Correct.

Hypothesis 6e: Students diagnosed with ADHD will not exhibit deficits in problem solving as measured by the D-KEFS Twenty Questions Test Total Weighted Achievement score.

Hypothesis 6f: Students diagnosed with ADHD will not exhibit deficits in planning as measured by the D-KEFS Tower Test Total Achievement Score. 


\section{CHAPTER 2}

\section{Method}

\section{Participants}

Study participants included a clinical, non-random sample of college students ( $\mathrm{n}=70$ ADHD, $\mathrm{n}=31$ ADHD/LD) who completed an ADHD or combined ADHD/LD assessment battery at a large university in the mid-Atlantic region between the dates of $8 / 1 / 2003$ to $3 / 1 / 2008$. The majority of students who presented for testing were between the ages of 18 and 24. Students paid $\$ 220$ for ADHD testing, and $\$ 320$ for combined LD/ADHD testing. Students typically sought ADHD/LD assessments for the purpose of receiving accommodations for their disability. As such, the testing was completed for diagnostic purposes. All participants completed an informed consent waiver. The informed consent waiver specified that the students' data may be used for research purposes following IRB approval. IRB determined that the research was exempt.

\section{Materials}

ADHD/LD Assessment Screening Questionnaire. This questionnaire obtained selfreport data of the student's specific academic difficulties (see Appendix A).

ADHD/LD Testing Background Information Form. This questionnaire obtained self-report data of the student's age, college major and year, GPA, academic history, and medical, familial, and psychosocial history (see Appendix B).

Clinical Interview. Each student was interviewed to further explore and clarify information regarding their academic, medical, familial, and psychosocial history for diagnostic purposes. The ADHD/LD Assessment Screening Questionnaire and the ADHD/LD Testing Background Information Form were used to guide the interview. 
Additional psychological inventories were administered as appropriate (i.e. Beck Depression Inventory, Beck Anxiety Inventory).

College ADHD Response Evaluation (CARE). The CARE is the first assessment system that was designed and normed exclusively for individuals at the university level (CARE; Glutting, Sheslow, \& Adams, 2002). The CARE encourages consensual validity because it includes co-normed student and parent assessments: the Student Response Inventory (CARE-SRI) and the Parent Response Inventory (CARE-PRI) (Frazier, Youngstrom, Glutting, \& Watkins, 2007). The CARE allows for a cross-informant, multidimensional evaluation of behaviors common to ADHD. The CARE self-report items were developed from two sources, which allow for multidimensional evaluation. The CARE is derived both from factor analytic studies with college students, and from clinical criteria from the DSM-IV. The CARE provides two frameworks for interpretation. The first allows for comparison with the average college student and was normed using a representative sample of college students across the U.S. The second framework provides for comparison with criteria used for the general population as specified in the DSM-IV. The results can be interpreted with reference to general national norms for college students, or to male or female gender specific norms. A matched sample of 1080 college students and their parents were selected for inclusion in the normative sample. Each student completed the CARE-SRI and one of their parents completed the CARE-PRI. The CARE standardization model accounted for ethnicity, gender, ability level, age-grade level, region of residence, and ADHD diagnosis.

College ADHD Response Evaluation - Student Response Inventory (CARE-SRI). 
The CARE-SRI is a 59 question self-report inventory designed to provide information relevant to the assessment of ADHD at the college level. Eighteen CARE-SRI items come directly from criteria in the DSM-IV. The CARE is relatively brief and requires an average of 5 to 15 minutes to complete. Mental health professionals (postsecondary disability service providers, college counselors, psychologists, psychiatrists) experienced in working with students with ADHD at the college level contributed to item development via interviews and recorded questions. For each CARE-SRI item, students indicate whether they agree, disagree, or are undecided about whether the item's content applies to their day-to-day life. The CARE-SRI generates percentile ranks on both DSMIV scales derived by comparison to DSM-IV diagnostic criteria (Inattention Scale, Hyperactive Impulsive Scale) and Factor Scales derived from research (Inattention Scale, Hyperactivity Scale, Impulsivity Scale, Total Score). The CARE-SRI provide scores based on results from factor analyses as well as scores founded on ADHD criteria in the DSM-IV. The CARE-SRI provides a total of six scores (factor-based Inattentiveness, factor-based Hyperactivity, factor-based Impulsivity; DSM-IV Inattentiveness, DSM-IV Hyperactivity; and a Total score). Percentiles are the primary derived score for interpretation on the CARE-SRI. However, raw scores can be transformed to percentiles and $\mathrm{T}$ scores $(\mathrm{M}=50, \mathrm{SD}=10)$.

Exploratory and confirmatory factor analyses with independent replications support the three factor-based scores from the CARE-SRI (Glutting et al., 2002). Studies examining external validity, including one that evaluated diagnostic validity between college students with ADHD and those without ADHD, are reviewed in the CARE manual (Glutting et al., 2002). Internal consistency reliabilities for the CARE-SRI norm 
sample were higher for the factor-based scales than the DSM-IV scales. For the factorbased scales, internal consistency was .82 for the Inattentiveness scale, .87 for the Hyperactivity scale, .77 for the Impulsivity scale, and .90 for the Total scale. These consistency scores are high and exceed the suggested minimum of .70. In contrast, for the DSM-IV scales, internal consistency was .63 for Inattentiveness and .65 for Hyperactivity/Impulsivity. These results suggest that the factor-based scores should receive greater consideration than DSM-IV scores for the CARE-SRI. Test-retest reliability coefficients are as follows: .88 for Inattentiveness, .87 for Hyperactivity, .77 for Impulsivity, .91 for Combined, .84 for DSM-IV Inattentiveness, and .81 for DSM-IV Hyperactivity/Impulsivity. These reliability coefficients demonstrate that scores on the CARE-SRI possess satisfactory (.77) to excellent (.91) stability (Glutting et al., 2002). College ADHD Response Evaluation - Parent Response Inventory (CARE-PRI). The CARE-PRI is a 46 item, objective rating scale, completed by the student's parent(s) to assess ADHD symptoms. The CARE-PRI was developed to supplement and enhance data supplied by students on the student report. The CARE-PRI uses the same item format as the student report, with parents indicating whether they agree, disagree, or are undecided about how an item's content applies to their child. The CARE-PRI also requires approximately 5 to 15 minutes to complete. The CARE-PRI asks parents to give an opinion about what their child was like when they were in elementary school (approximately 5 to 8 years old). This information can aid examiners in clarifying whether a student's difficulties are persistent or whether they are a reaction to more recent stressful events. The CARE-PRI provides the parent's view, which is compared with the ratings of other parents of college students in the norm group. The CARE-PRI 
provides DSM-IV and Factor scales as described above for the CARE-SRI. The CAREPRI provides scores based on results from factor analyses as well as scores founded on ADHD criteria in the DSM-IV. The CARE-SRI provides a total of five scores (factorbased Inattentiveness, factor-based Hyperactivity/Impulsivity, DSM-IV Inattentiveness, DSM-IV Hyperactivity, and a Total score). Percentiles are the primary derived score for interpretation on the CARE-PRI. However, raw scores may be transformed to percentiles and $\mathrm{T}$ scores $(\mathrm{M}=50, \mathrm{SD}=10)$.

The two factor-based scores from the CARE-PRI were supported through exploratory and confirmatory factor analyses (Glutting et al., 2002). Studies examining external validity are presented in the CARE manual. Similar to the CARE-SRI, internal consistency reliabilities for the CARE-PRI norm sample were higher for the factor-based scales than the DSM-IV scales. However, reliabilities for the two DSM-IV scales and the factor based scales both exceeded the .70 criterion. For the factor-based scales, internal consistency was .85 for the Inattentiveness scale, .86 for the Hyperactivity scale, and .89 for the Total scale. For the DSM-IV scales, internal consistency was .79 for Inattentiveness and .74 for Hyperactivity/Impulsivity. These results suggest that both the factor-based scores and DSM-IV scores can be used with confidence. Test-retest reliabilities were not available for the CARE-PRI.

Wechsler Adult Intelligence Scale - Third Edition (WAIS-III). The WAIS-III (Wechsler, 1997), the latest version of the Wechsler scales for adults, was published in 1997. The WAIS-III was designed for adults' ages 16 to 89 years, and standardized on an American sample of 2,450 adults. The sample was divided into 13 age bands and stratified along demographic variables, including age, sex, race, education level, and 
geographic region according to the U.S. census data. The WAIS-III contains 14 subtests, with seven subtests in the Verbal scale and seven in the Performance Scale. The Verbal scale is comprised of the following subtests: Vocabulary, Similarities, Arithmetic, Digit Span, Information, Comprehension, and Letter-Number Sequencing. The Performance scale consists of: Picture Completion, Digit-Symbol Coding, Block Design, Matrix Reasoning, Picture Arrangement, Symbol Search, and Object Assembly. Object Assembly is a supplementary subtest. The WAIS-III also delineates index scores according to the following: Verbal Comprehension (Vocabulary, Similarities, Information), Perceptual Organization (Picture Completion, Block Design, Matrix Reasoning), Working Memory (Arithmetic, Digit Span, Letter-Number Sequencing), and Processing Speed (Coding, Symbol Search).

The derivation of subtest scaled scores included conversion of each age group's subtest raw scores into percentiles and then to a scale with a mean of 10 and a standardization deviation of 3. Cumulative frequency distributions of raw scores for each age group were normalized and any minor sampling fluctuations were smoothed. The sums of subtest scaled scores were formed by calculating the means and standard deviations of the sums of scaled score distributions for each group. Analysis of variance did not reveal statistically significant variation by age group in the mean scores for the scales and indices. Thus, the age groups were combined. This resulted in the conversion of the distribution of the sums of scaled scores to a scale with a mean of 100 and a standard deviation of 15 .

Evidence of the reliability of the WAIS-III is reviewed in the WAIS-III Technical Manual (Wechsler, 1997). Test reliability refers to the accuracy, consistency, and 
stability of test scores across situations. The average reliability coefficients of most of the WAIS-III subtests (except Picture Arrangement, Symbol Search, and Object assembly) range from .82 to .93 (Wechsler, 1997). Coefficients are extremely high ( $\geq .90)$ for the Vocabulary, Digit Span, Information, and Matrix Reasoning subtests. The Arithmetic, Comprehension, Letter-Number Sequencing, Picture Completion, Digit-Symbol Coding, Similarities, and Block Design subtests have coefficients ranging from .82 to .88 . An average test-retest coefficient of .77 was found for Symbol Search, which is relatively high for test-retest reliability. Picture Arrangement and Object Assembly subtests have lower coefficients, .74 and .70 , respectively. The Object Assembly subtest was excluded from the computation of IQ and Index scores based on its low reliability.

The average reliability coefficients for the WAIS-III IQ and index scales are generally higher than those of the individual subtests, and range from .88 to .97 . This difference is explained as occurring because each subtest represents only a small part of an individual's entire intellectual functioning. The IQ and index scores summarize the individual's performance on a wider range of abilities. Thus, higher reliability coefficients for the WAIS-III IQ scores and indexes are expected. The WAIS-III Processing Speed index score $(r=.88)$ has somewhat lower reliability coefficients. This is expected based on the relatively small number of subtests (Coding and Symbol Search) composing this index. The WAIS-III IQ and index scores have excellent reliability. The Full Scale, Verbal IQ, and Performance IQ scales have internal consistency reliability coefficients ranging from .93 to .98 , across all age groups. The average internal consistency reliability coefficients, based on the 13 age groups, are .98 for the Full Scale IQ, .97 for the Verbal Scale IQ, and .94 for the Performance Scale IQ (Sattler, 2002). 
Test-retest reliability consisted of re-testing after a 2 to 12 week period, with a mean of 34.6 days. A total of 394 adults, evenly divided into 13 age groups, showed that the WAIS-III scores possess adequate stability across time and for all age groups (Wechsler, 1997). Full Scale and Verbal IQ scores are somewhat more stable than Performance IQ scores. For test-retest stability coefficient statistical analysis, scores were calculated for four pooled age groups. The stability coefficients were $.91, .96, .96$, and .96 for the Full Scale IQ, $.91, .95, .97$, and .95 for the Verbal IQ, and $.83, .88, .91$, and .93 for the Performance IQ. The stability coefficients for the subtests ranged from a low of .48 for Letter-Number Sequencing at 16 to 29 years to a high of .94 for Information at 75 to 89 years. Overall, the WAIS appears to show adequate concurrent, criterion, and construct validity (Wechsler, 1997).

Criterion-related validity refers to the relationship between test scores and some type of criterion or outcome, such as ratings or classification (Sattler, 2001). A study with 192 adults examining the relationship between the WAIS-III and WAIS-R is reviewed in the WAIS-III Technical Manual (1997). The two measures revealed similar scale and subtest scores. The correlation coefficients for the sample are $.94, .86$, and .93 for the VIQ, PIQ, and FSIQ scores, respectively, suggesting that the WAIS-III measures essentially the same construct as does the WAIS-R. The Full Scale IQ, VIQ, and PIQ scores were 2.9, 1.2, and 4.8 points lower on average for the WAIS-III than the WAIS-R, respectively. Correlation coefficients between the WAIS-III and WISC-III IQ scores were also calculated, and found to be very high and statistically significant. The correlation coefficients were as follows: $.88, .78$, and .88 for the VIQ, PIQ, and FSIQ scores, respectively (Wechsler, 1997). In addition, a correlation coefficient of .88 was 
found between the WAIS-III FSIQ and the Stanford-Binet Intelligence Scale-Fourth Edition composite score.

Construct validity is defined as the extent to which the test measures the psychological construct of interest (Sattler, 2001). Overall, there is support for the construct validity of the WAIS-III. Results from factor analyses support that the test measures the construct it was designed to measure (Wechsler, 1997). The results from most of the exploratory and confirmatory factor analysis support that the four-factor model (Verbal Comprehension, Perceptual Organization, Working Memory, Processing Speed) best fits the data for the total sample (Wechsler, 1997). Hierarchical regression analysis indicated that three subtests are sufficient to adequately measure the ability tapped by the indexes. Further, Sattler's (2002) factor analyses of the WAIS-III standardization sample indicated that all 14 subtests measure $(\mathrm{g})$ or general intelligence with a moderate or high degree of success. The pattern of intercorrelations also provided evidence of convergent and discriminate validity.

The WAIS-III provides an estimate of an individual's IQ. The Verbal IQ, Performance IQ, Full Scale IQ, Verbal Comprehension Index, Perceptual Organization Index, Working Memory Index, and Processing Speed Index scores have means of 100 and standard deviations of 15 points. An IQ score of 100 defines the performance of the average adult. Subtest scores have a mean of 10 and a standard deviation of 3 . The WAIS-III also allows for the identification of discrepancy scores as compared to the normative population or the individual's peer group. The WAIS-III Technical Manual (Wechsler, 1997) provides a complete review of the psychometric properties of all composite indices and subtests. For ease of interpretation, subtest scores will be presented 
in the standard manner, as age corrected scale scores with a mean of 10 and standard deviation of 3 , and factor indices are standardized with a mean of 100 and standard deviation of 15 .

Integrated Visual \& Auditory Continuous Performance Test (IVA). The IVA CPT is an integrated 13 minute test designed to aid in the diagnosis and quantification of ADHD symptoms. The IVA combines two types of CPTs for both visual and auditory modalities and uses periods of frequent responding and non-frequent responding, designed to assess response control and attention (Sandford \& Turner, 2000). The test administration is computerized, and involves responding or inhibiting a response to counter-balanced auditory and visual stimuli for 500 trials, 1.5 seconds in length. The test demands constant, sustained attention for adequate performance (Sandford \& Turner, 2000). The test requires students to click a computer mouse only when a "1" is seen or heard and not to click when a " 2 " is seen or heard. Scores are reported with a mean of 100 and a standard deviation of 15 . Performance on the IVA may fluctuate as it can be influenced by motivational, emotional, and physical states.

The IVA consists of 22 subscales that provide information regarding inattention, inhibition, response consistency, variability in attention, and overall speed of discriminating reaction time. The IVA can be administered to individuals ages 5 to adulthood. The test is intended to be mildly boring and to produce errors of commission (impulsivity/response inhibition) and omission (inattention). The IVA also provides two global scales, the Full Scale Response Control Quotient (FSRCQ) and the Full Scale Attention Quotient (FSAQ). The FSRCQ provides a measure of commission and impulsivity. It is comprised of scores from Prudence (impulsivity and response 
inhibition), Consistency (reliability of response time and staying on task), and Stamina (sustained attention and effort over time). The FSAQ measures omission and attending errors, and is comprised of scores from Vigilance (inattention), Focus (speed of mental processing), and Speed (reaction time). Each of the above scores are reported separately for both auditory and visual modalities, and again as a combined score. The IVA was also developed to aid in differentiating ADHD subtypes (Sandford \& Turner, 2000).

The IVA manual presents evidence of reliability and validity. A test re-test reliability study was conducted with 70 individuals without identified attention, learning, neurological, psychological, or self-control problems. Significant correlations for testretest scores (.37 to .75) were found for all IVA composite quotients, demonstrating stability of the IVA over time. Moderately strong positive relationships were found for the response control quotients, and the attention quotient score correlations demonstrated very strong positive relationships (Sandford \& Turner, 2000). Twenty of the 22 IVA scale raw scores had significant positive relationships, eighteen of which showed a moderately strong to very strong relationship (.46 to .88 ). The authors suggest that these results substantiate the CPT as a significantly stable measure of performance both globally and with specific scales.

Evidence of concurrent and diagnostic validity of the IVA are also presented in the IVA manual. A group consisting of 26 children, ages 7 to 12 years old, who were diagnosed as having ADHD comprised the first group. The second group, comprised of 31 children, ages 7 to 12 years old, except for one 15 year old, were included as a "normal" control group. Criteria for the control group included no indication of neurological, learning, emotional, or ADHD related problems based on parental report. 
Instruments for comparison included the IVA, the Test of Variables of Attention (TOVA) CPT, the Gordon CPT, the CPRS-39 ADHD rating scale, and the Children's Attention Score (CAS). Comparison of the accuracy of the IVA to diagnoses made by a physician or psychologist who had independently evaluated the child previously also provided diagnostic discriminate validity. The IVA's overall accuracy was found to be significant $(\mathrm{p}<.0001)($ Sandford \& Turner, 2000).

Results suggest that the IVA has sufficient sensitivity (92\%) (proportion of ADHD children who are found positive on the measure). The positive predictive power (the proportion of test negatives that do not have ADHD) was 93\%. The specificity (proportion of non-ADHD children who received a negative finding on the measure) of the IVA was $90 \%$. The authors note that the IVA had the lowest rate of false negatives among various CPT test instruments. Further, concurrent validity was examined by comparing the children identified as ADHD by the IVA to children identified as ADHD by the other diagnostic instruments. The percent of agreement was as follows: $90 \%$ for the TOVA, $100 \%$ for the Gordon, $92 \%$ for the CPRS-39, and $100 \%$ for the CAS.

The comparative accuracy of the IVA and various other diagnostic instruments was assessed by examining false negative rates with the criterion reference of the prestudy clinical diagnosis. The percent of false negatives were as follows: $7.7 \%$ for the IVA, $12.5 \%$ for the TOVA, $36.0 \%$ for the Gordon, $45.5 \%$ for the CPRS-39, and $59.1 \%$ for the CAS. Sandford and Turner (2000) conclude that validity research substantiates the IVA as an accurate cognitive test which can provide important objective data for inclusion in the assessment of ADHD. 
Delis-Kaplan Executive Function System (D-KEFS). The Delis-Kaplan Executive Function System (D-KEFS; Delis, Kaplan, \& Kramer, 2001) provides a comprehensive collection of standardized tests for the assessment of executive functions in children, adolescents, and adults between the ages of 8 and 89 . The D-KEFS provides the first set of tests normed on a large and representative national sample designed exclusively for the assessment of executive functions, including abstract thinking, concept formation, creativity, flexibility of thinking, impulse control, inhibition, planning, and problem solving, in both verbal and spatial modalities. An objective in designing the D-KEFS was to provide psychologists with a comprehensive battery of tests to assess the complex and multifactorial domains of frontal lobe functioning (Homack, Lee, \& Riccio, 2005). The national standardization study included over 1700 children and adults, from ages 8 to 89, selected to match the demographic characteristics of the US population. There are a total of nine tests comprising the D-KEFS, which can be used individually or in conjunction with the other measures of executive functioning. The tests measure a wide array of verbal and nonverbal executive functions.

The D-KEFS employs a "cognitive-process approach," which allows for assessment of the component functions of higher-level cognitive tasks. The D-KEFS allows for isolation and measurement of two types of component processes: fundamental cognitive skills on which the higher-level executive functions depend (i.e. attention, perception, language), and various higher-order cognitive functions (i.e. concept formation, inhibition, planning, and cognitive flexibility) that may contribute to successful task execution. These component process scores aid in illuminating the diverse 
nature of the cognitive skills required for successful task execution, and in identifying the mechanisms underlying impairment (Homack, Lee, \& Riccio, 2005).

The D-KEFS is composed of nine tests that are either relatively new tests, or adaptations of tests that have been used in the past. The following nine stand-alone tests can be administered individually or as a group: Trail Making Test, Verbal Fluency Test, Design Fluency Test, Color-Word Interference Test, Sorting Test, Twenty Questions Test, Word Context Test, Tower Test, and Proverb Test. D-KEFS test includes primary and optional measures and provides between 6 and 34 scores. In addition, five D-KEFS tests provide several primary or optional "contrast" measures. Raw scores are converted to scaled scores, correcting for 16 separate age groups. Scaled scores have a mean of 10 and a standard deviation of 3. Raw scores for measures that have limited ranges in the normative group are converted to cumulative percentile ranks. Normative scores are derived for each of the tests. Factor analytic techniques were not applied for the D-KEFS. Thus, empirically derived index scores or factor scores are not available.

The D-KEFS was standardized on a nationally representative, stratified sample of 1750 non-clinical children, adolescents, and adults, ages 8 to 89 years. The D-KEFS normative sample was based on the 2000 U.S. Census. Age, geographic region, race/ethnicity, sex, and years of education were considered for population proportionate sampling. A minimum of 75 people were included in each of the 16 age groups to generate age-specific norms. The proportions of men and women and racial/ethnic groups at each age group were stratified to approximate the U.S. census estimates. Five educational groups, as delineated by the U.S. Census, were used for the D-KEFS sample: less than or equal to 8 years of education, 9-11 years, 12 years, 13-15 years, and 16 or 
more years. More than 150 sites, across the following four geographic regions, as defined by the 2000 U.S. Census data were used in the standardization study: Northeast, North Central, South, and West. The D-KEFS standardization sample includes only non-clinical samples. Individuals were excluded from the standardization study if they endorsed one or more medical or psychiatric condition that might impact performance on cognitive tests (Homack, Lee, \& Riccio, 2005).

Evidence of reliability is presented in the D-KEFS Technical Manual (Delis, Kaplan, \& Kramer, 2001). Internal consistency, test-retest reliability, standard errors of measurement, and confidence intervals are provided for each D-KEFS test. Test-retest reliability coefficients were reported using a sample of 101 examinees, similar in composition to the standardization sample. Overall, split-half reliability estimates varied significantly across tests, conditions within tests, and age groups. Many of the overall achievement scores of the tests have adequate to good reliability coefficients, whereas some of the optional process measures have lower reliabilities. See the technical manual (Delis, Kaplan, \& Kramer, 2001) for alternate-form validity and reliability estimates.

Evidence of validity is also presented in the D-KEFS Technical Manual. Specifically, correlations between measures of different D-KEFS tests, correlations of DKEFS tests with other cognitive tests, intercorrelations of measures within individual DKEFS tests, and findings from pilot studies with clinical populations are presented. Relatively low positive correlations were found for total achievement scores from the different D-KEFS tests. In regards to intercorrelations, the authors suggest that they were generally consistent with the expected pattern of positive and negative correlations for most D-KEFS tests. However, the direction and strength of associations between tests 
varied considerably depending on age groups (Homack, Lee, \& Riccio, 2005). Validity studies with clinical populations suggest that the D-KEFS tests have reasonable sensitivity in distinguishing several different types of clinical groups from normal controls, including individuals with chronic alcoholism, fetal alcohol exposure, focal frontal lesions, Parkinson's disease, and Schizophrenia. Additional evidence of reliability and validity can be found in the D-KEFS Technical Manual (Delis, Kaplan, \& Kramer, 2001).

In a review, Baron (2004) points out several current limitations of the D-KEFS. Baron suggests that evidence of reliability and validity contributing to an understanding of the utility of each test, combinations of tests, or of the value of optional indices is not yet sufficient in the literature. This limitation may be lessened as the literature emerges. Homack, Lee, and Riccio (2005) also comment that there are many measures in the DKEFS ready for clinical use, while others require additional research to support their clinical utility. Both Baron (2004) and Homack et al. (2005) emphasize that additional research is necessary to fully establish the psychometric properties of the D-KEFS.

D-KEFS Trail Making Test (TMT). The main task of the D-KEFS TMT is number-letter switching (cognitive flexibility) on a visual-motor sequencing procedure. The fundamental skills measured with the TMT include visual scanning, number sequencing, letter sequencing, and motor speed. The internal consistency coefficients for the Total Score range from moderate to high (.57 to .81$)$. Test-retest reliability results, combined for all ages range from .38 for the switching condition to .77 for motor speed. The authors stated that most total score correlations fell in the moderate range. 
D-KEFS Verbal Fluency Test. The D-KEFS Verbal Fluency Test was adapted from the Controlled Oral Word Association Test. There are three main tasks. The first is a Letter Fluency task in which individuals are instructed to say words that begin with a specified letter as quickly as possible in three trials of 60 seconds each. Category Fluency involves saying words that belong to a designated semantic category as quickly as possible in two trials of 60 seconds each. The third condition, Category Switching requires alternating between saying words from two different semantic categories as quickly as possible for 60 seconds. Executive functions measured include the ability to generate words fluently in an effortful, phonemic format (letter fluency), from overlearned concepts (category fluency), and while simultaneously shifting between overlearned concepts (category switching). The internal consistency coefficients across all age groups are as follows: Letter Fluency (.68 to .90), Category Fluency (.53 to .76), Category Switching Total Correct (.37 to .68), and Category Switching Total Switching (.51 to .76). Test-retest reliability results, combined for all ages range from .36 for Category Switching Total Switching to .80 for Letter Fluency.

D-KEFS Design Fluency Test. For the D-KEFS Design Fluency Test, the examinees is presented with rows of boxes with dots that they must connect with four lines only to make different designs. The boxes in the first condition contain filled dots only. The boxes in the second and third conditions contain filled dots and empty dots. For the second condition, the examinee must use only the empty dots. For the third condition, the examinee must alternate between empty and filled boxes. The D-KEFS Design Fluency test is a nonverbal task analogous to verbal fluency which measures mental shifting errors and perseverative responding. The authors state that item interdependence 
preclude the use of internal consistency procedures. Test-retest reliability results, combined for all ages were low (.32) for the Switching condition, moderate (.58) for Correct Designs Filled Dots Only, and moderate (.57) for Correct Designs Empty Dot Only.

D-KEFS Color-Word Interference Test. The D-KEFS Color-Word Interference Test, adapted from the Stroop test, contains four conditions. The first condition involves basic naming of color patches and the second involves basic reading of words that denote colors printed in black ink. The third condition requires the examinee to inhibit reading the words denoting colors in order to name the dissonant ink colors in which those words are printed. The final condition requires switching back and forth between naming the dissonant ink colors and reading the conflicting words. The EFs measured include inhibition of a more automatic verbal response (reading) in order to generate a conflicting response of naming the dissonant ink colors, and cognitive flexibility. The internal consistency coefficients ranged across all age groups from .62 to .86 for the Combined Score. Test-retest reliability results, combined for all ages range from .62 (Word Reading) to .75 (Inhibition).

D-KEFS Sorting Test. An adaptation from the California Card Sorting Test, the D-KEFS Sorting Test employs two conditions. Free Sorting requires the examinee to sort cards based on as many different concepts as possible and to describe the concepts employed. Sort Recognition requires the examinee to identify and describe the concepts used to generate sorts as presented by the examiner. Concept formation, conceptual reasoning, problem solving and the ability to initiate problem solving are executive functions tapped by this test. The internal consistency coefficients ranged from .62 to .81 
across the age groups of 8 to 89 years. Test-retest reliability results, combined for all ages range from .50 to .60 .

D-KEFS Twenty Questions Test. The D-KEFS Twenty Questions Test requires examinees to ask the fewest number of yes/no questions in order to identify the unknown target object on a page with pictures of 30 objects. Executive functions required for this test include concept formation, problem-solving, and abstract thinking. Particularly, this task requires the ability to identify the various categories and subcategories represented in the 30 objects and to formulate abstract, yes/no questions that eliminate the maximum number of objects regardless of the examiner's answer. The internal consistency coefficients ranged from .10 for age group 16 to 19 years to .55 for age group 70 to 79 years for the Total Weighted Achievement score. The coefficients for the Initial Abstraction score ranged from .72 (age group 9 years) to .87 (age group 15 years and 70 to 79 years). Test-retest reliability results, combined for all ages was .24 for the Total Weighted Achievement score to .43 for the Initial Abstraction Score.

\section{D-KEFS Word Context Test. The D-KEFS Word Context Test requires} examinees to discover the meaning of made-up words based on clues given in sentences. Deductive reasoning, integration of multiple bits of information, hypothesis testing, and flexibility of thinking are the executive functions measured by this test. The internal consistency coefficients ranged from .47 (age group 13 years) to .72 (age group 50 to 59 years). Test-retest reliability results, combined for all ages was .70 for Total First Trial Consistently Correct.

D-KEFS Tower Test. For the D-KEFS Tower Test, examinees must move disks across three pegs to build the target tower in the fewest number of moves possible, 
moving only one disk at a time and never placing a larger disk over a smaller disk. Executive functions required for this test include spatial planning, rule learning, inhibition of impulsive responding, inhibition of perseverative responding, and establishing and maintaining the instructional set. The internal consistency coefficients ranged from .43 (age group 14 years) to .84 (age group 10 years). Test-retest reliability results, combined for all ages was .44 for the Total Achievement score.

D-KEFS Proverb Test. There are two conditions in the D-KEFS Proverb Test. The Free Inquiry condition requires examinees to interpret proverbs orally without assistance or cues. The Multiple Choice condition requires examines to select the best interpretation among the four presented. Verbal and novel abstraction skills are required for this test. The internal consistency coefficients ranged from .68 (age group 16 to 19 years) to .81 (age group 60 to 69 years). Test-retest reliability results, combined for all ages was .76 for the Free Inquiry Total Achievement Score.

\section{ADHD Classification}

Data from the clinical interview, IVA, CARE-SRI, CARE-PRI, WAIS-III, and DKEFS were taken into consideration in determining if a diagnosis of ADHD is appropriate. Students were diagnosed with ADHD according to the following criteria: IVA, CARE-SRI, and CARE-PRI (or comparable data) scores consistent with ADHD, presence of cognitive deficits as indicated by the WAIS-III or D-KEFS scores, evidence of functional impairment, and corroborating information obtained by the clinical interview. A diagnosis of LD was determined if an individual's achievement (reading, math, written language), as indicated by the WJ-III, fell at least one standard deviation below their IQ as determined by the WAIS-III. 


\section{Research Design}

Students interested in LD/ADHD testing first consulted with a senior staff member (one of two Assessment Coordinators) at the university clinic to plan the appropriate assessments. Students then completed a demographic information form, notice of privacy practices, and informed consent prior to the testing session. The assessment examiner reviewed informed consent with each student on the day of testing. The assessment batteries were administered by one of nineteen psychology interns and licensed psychologists, over the course of five years. Each battery was reviewed and signed by one of two Assessment Coordinators, both experienced licensed psychologists. Assessment for ADHD included the following: clinical interview, WAIS-III, CARE-SRI, IVA, and D-KEFS. Students being assessed for both LD and ADHD also completed the Bender Gestalt Test of Visual-Motor Integration and WJ-III tests of Achievement. With the student's permission, CARE-PRI forms were also mailed to a parent (or person familiar with the student's behavior during childhood) to complete. If this not was possible, additional data regarding the student's behaviors during childhood was requested (i.e. report cards, teacher behavior rating forms, specific examples as identified by self-report). Five hours was the average time required to complete the battery of measures used to assess for ADHD. Approximately three additional hours were needed for completing a combined LD/ADHD assessment. Students being treated with stimulant medication were required to abstain from medication use on the day of testing.

Students who had all of the study variables available, and who had a diagnosis of ADHD or ADHD and a comorbid LD were included in the study. Specifically, data from the CARE-SRI, CARE-PRI, IVA, WAIS-III, and D-KEFS was needed for analysis, as 
well as gender, age, level of education, and diagnostic information as determined by the assessment. Students with comorbid disorders other than a learning disorder (i.e. mood disorder, anxiety disorder) were excluded from the study.

For data entry and analysis, each student was assigned a number. To assure confidentiality, only this number was connected to the research data. The frequencies and percentages of demographic information for all the participants in the study were computed. The following student characteristics are displayed: age, sex, ethnicity, student status, high-school grade point average, current grade point average, SAT scores, CARE-SRI and CARE-PRI scores, and diagnosis determined following formal testing. The Independent Variable for this study was diagnosis (ADHD, ADHD and LD). The Dependent Variables included scores from the IVA, WAIS-III, and D-KEFS.

Research question one was assessed by conducting four one sample $t$-tests comparing WAIS-III index scores for students diagnosed with ADHD with the WAIS-III standardization sample to investigate whether there were differences for group on the WAIS-III index scores. Research question two was assessed by conducting two one sample $t$-tests comparing IVA scores for students diagnosed with ADHD with the IVA standardization sample to investigate whether there were differences for group on the IVA scores. The $t$-tests for research questions one and two were conducted as two-tailed tests with an alpha level of .05. A Bonferroni correction was applied to data analysis procedures in order to control for the multiple t-tests.

The third research question was assessed with a MANOVA, comparing the WAIS-III index scores for students diagnosed with ADHD as compared to students diagnosed with ADHD and a comorbid LD to investigate whether there were differences 
for group on the WAIS-III index scores. To assess research question four, a MANOVA was used to compare the IVA scores for students diagnosed with ADHD as compared to students diagnosed with ADHD and a comorbid LD to investigate whether there were differences for group on the IVA scores. The MANOVA's were not significant for research questions three or four, thus individual analyses of variance (ANOVA) were not run to assess the significance of each dependent variable. All alpha levels were set at ( $p$ $=.05)$. A Bonferroni correction was applied to data analysis procedures for the third and fourth research questions in order to control for multiple comparisons. Chi Square was run to determine if the distribution of gender and differed between the two groups. Eta squared $\left(\eta^{2}\right)$, the proportion of variance in the dependent variable that is accounted for by group membership (i.e. ADHD, ADHD and LD) and observed power, the probability of finding a statistically significant effect if an effect is truly present were reported.

The fifth research question was assessed by calculating the differences between the WAIS-III index scores (VCI/POI, VCI/WMI, VCI/PSI, POI/WMI, POI/PSI, WMI/PSI) for the ADHD sample. Next, the differences were examined for statistical significance at the $\mathrm{p}<.05$ level according to the appropriate age specific tables found in Appendix B, Table B.1 of the WAIS-III Administration and Scoring Manual. Given that a statistically significant difference in index scores may not represent a clinically significant difference, the frequency of occurrence in the standardization sample according to tables found in Sattler (2001) Appendix C, Tables C-4 and C-5 were also considered. Sattler (2001) suggests that differences between scores that occur in less than $15 \%$ of the standardization sample should be judged as unusual. A cut off score of less than $15 \%$ was used in the calculation of percentages for the study sample. 
Research question six was assessed with descriptive statistics. The percentage of students exhibiting executive functioning deficits (greater than or equal to one standard deviation below the standardization sample mean) in the domains of cognitive flexibility, concept formation, verbal and non-verbal fluency, planning, and problem solving were presented. Deficits in executive functioning were determined based on an idiographic review of each student's scores. For D-KEFS tests that provide contrast scores (Trail Making Test, Verbal Fluency, Design Fluency, Color-Word Interference, and Sorting Test), a deficit in executive functioning were substantiated only if the contrast scores indicate a deficit in executive functioning, over and above deficits in component or fundamental skills measured by the test.

Additional descriptive statistics were also conducted to ascertain the percentage of students exhibiting executive functioning strengths (greater than or equal to one standard deviation above the standardization sample mean) in the domains of cognitive flexibility, concept formation, verbal and non-verbal fluency, planning, and problem solving. Strengths in executive functioning were determined based on an idiographic review of each student's scores. For D-KEFS tests that provide contrast scores (Trail Making Test, Verbal Fluency, Design Fluency, Color-Word Interference, and Sorting Test), a strength in executive functioning was substantiated only if the contrast scores indicate a strength in executive functioning, over and above strengths in component skills measured by the test. In addition, the means and standard deviations for measures of executive functioning were also reported. 


\section{CHAPTER 3}

Results

The purpose of the current study was to examine the neuropsychological functioning of college students with ADHD. A total of 128 students completed testing for ADHD/LD between August of 2003 and March of 2008. Seventy participants received a diagnosis of ADHD, and thirty-one were diagnosed with ADHD and a comorbid LD. Students who did not receive a diagnosis of ADHD and students who exhibited comorbidity other than LD $(n=27)$ were excluded from the study.

The ADHD student group consisted of participants with IVA, CARE-SRI, and CARE-PRI (or comparable data) scores consistent with ADHD, presence of cognitive deficits as indicated by the WAIS-III or D-KEFS scores, evidence of functional impairment, and corroborating information obtained by the clinical interview. The ADHD/LD student group consisted of participants who met criteria for both ADHD and LD. A diagnosis of LD was determined if an individual's achievement (reading, math, and/or written language), as indicated by the Woodcock-Johnson-III, fell at least one standard deviation below their IQ as determined by the WAIS-III.

\section{Demographic Characteristics}

The age distribution of the 70 ADHD student sample was as follows: (a) 40 (57.1\%) were within the 18 to 19 age range, (b) 17 (24.3\%) were within the 20 to 21 age range, (c) $9(12.9 \%)$ were within the 22 to 24 age range, and (d) $4(5.7 \%)$ were in the 25 and above age range. The age distribution of the $31 \mathrm{ADHD} / \mathrm{LD}$ sample was as follows: (a) $8(25.8 \%)$ were within the 18 to 19 age range, (b) 14 (45.2\%) were within the 20 to 21 
age range, (c) $6(19.4 \%)$ were within the 22 to 24 age range, and (d) $3(9.7 \%)$ were in the 25 and above age range.

The gender distribution was comparable for the ADHD and ADHD/LD groups. The ADHD group consisted of 38 (54.3\%) men and $32(45.7 \%)$ women, and the ADHD/LD group included $18(58.1 \%)$ men and 13 (41.9\%) women. The majority of participants were Caucasian. The race distribution of the ADHD sample was as follows: (a) 60 (85.7\%) Caucasian, (b) 1 (1.4\%) African American/Black, (c) 2 (2.9\%) Biracial, (d) $3(4.3 \%)$ Asian, and (e) 4 (5.7\%) Hispanic/Latino. The race distribution of the ADHD/LD group included: (a) 27 (87.1\%) Caucasian, (b) 1 (3.2\%) African American/Black, and (c) 3 (9.7\%) Hispanic/Latino.

Students in the sample spanned all years of college. In regards to year of college for the ADHD sample, 30 (42.9\%) of the students were Freshman, 19 (27.1\%) were Sophomores, 9 (12.9\%) were Juniors, 10 (14.3\%) were Seniors, and 2 (2.9\%) were pursuing a Graduate/Professional degree. The grade distribution of the ADHD/LD sample included 9 (29\%) Freshman, 5 (16.1\%) Sophomores, 10 (32.3\%) Juniors, 6 (19.4\%) Seniors, and $1(3.2 \%)$ Graduate/Professional. The majority of students in the current sample were diagnosed with ADHD-C. The ADHD subtype distribution of the ADHD group was as follows: 10 (14.3\%) ADHD-I, 1 (1.4\%) ADHD-HI, 47 (67.1) ADHD-C, and 12 (17.1) ADHD-NOS. The ADHD subtype distribution of the ADHD/LD group was as follows: 8 (27.6\%) ADHD-I, 2 (6.9\%) ADHD-HI, 12 (41.4) ADHD-C, and 9 (24.1) ADHD-NOS (See Table 2).

The average grade point average (GPA) for the ADHD sample was 2.23 $(\mathrm{SD}=0.84)$, and the average GPA for the ADHD/LD sample was $2.57(\mathrm{SD}=0.80)$. The 
average high-school GPAs for both samples was slightly higher with an average GPA of $3.04(\mathrm{SD}=0.53)$ for the ADHD sample, and $2.97(\mathrm{SD}=0.6)$ for the ADHD/LD sample. The average Standardized Achievement Test score was 1091 ( $\mathrm{SD}=126)$ for the ADHD sample and $1005(\mathrm{SD}=98)$ for the ADHD/LD sample.

The Full Scale Intelligence Quotient (FSIQ) scores for both groups fell in the Average range. The mean FSIQ score was $107(\mathrm{SD}=10.3)$ for the ADHD group and 107 $(\mathrm{SD}=8.1)$ for the ADHD/LD group. A one-sample $t$-test analysis conducted on the FSIQ for the ADHD group yielded a significant group difference, indicating that college students with ADHD have significantly higher FSIQ scores than the WAIS standardization sample; $t(68)=5.404, \mathrm{p}<.000$. The mean Verbal Intelligence Quotient (VIQ) and Performance Intelligence Quotient (PIQ) scores were also in the Average range. The mean VIQ score was $107(\mathrm{SD}=11.4)$ for the ADHD group and $107(\mathrm{SD}=8.3)$ for the ADHD/LD group. The mean PIQ scores were 105 (SD=11.0) for the ADHD sample and 106 (10.3) for the ADHD/LD group.

The College ADHD Response Evaluation - Student Response Inventory (CARESRI) and the College ADHD Response Evaluation - Parent Response Inventory (CAREPRI) mean percentile scores ranged from a minimum of 81.6 to a maximum of 94.9 for the ADHD group, and ranged from 81.0 to 96.4 for the ADHD/LD group (see Table 3). Research Questions

Research Question 1: Do students diagnosed with ADHD exhibit deficits in cognitive functioning as compared to the WAIS-III standardization sample? (Measured by the WAIS-III) Hypothesis 1: There will be no statistically significant difference in the WAIS-III IQ index scores (Verbal Comprehension Index (VCI), Perceptual Organization 
Index (POI), Working Memory Index (WMI), and Processing Speed Index (PSI)) obtained for the ADHD group when compared with the WAIS-III standardization sample. Research question one was assessed by conducting four one sample $t$-tests using the independent variable of ADHD diagnosis and the dependent variables of VCI, POI, WMI, and PSI. The $t$-tests were conducted as two-tailed tests with an alpha level of .05 , with a Bonferroni correction $(.05 / 4=.0125)$ for multiple $t$-tests. The $t$-test analysis conducted on the VCI yielded a significant group difference between college students with ADHD and the WAIS standardization sample; $t(67)=6.124, \mathrm{p}<.000$, thus rejecting the null hypothesis of no difference. Students with ADHD (M=108.99, SD=12.10) had significantly higher VCI scores than did the WAIS standardization sample (M=100, $\mathrm{SD}=15)$. A Cohen's d of 0.6597 was calculated indicating a medium effect size. Approximately $31 \%$ of the variability in the VCI score was accounted for.

The $t$-test analysis conducted on the POI yielded a significant group difference between college students with ADHD and the WAIS standardization sample; $t(67)=$ 5.781, $\mathrm{p}<.000$, thus rejecting the null hypothesis of no difference. Students with ADHD $(\mathrm{M}=109.18, \mathrm{SD}=13.09)$ had significantly higher POI scores than did the WAIS standardization sample $(\mathrm{M}=100, \mathrm{SD}=15)$. A Cohen's d of 0.6521 was calculated indicating a medium effect size. Approximately $31 \%$ of the variability in the POI score was accounted for.

The $t$-test analysis conducted on the WMI did not yielded a significant group difference between college students with ADHD and the WAIS standardization sample; $t$ $(66)=1.643, p=.105$, thus supporting the null hypothesis of no difference. Students with 
ADHD $(M=102.37, \mathrm{SD}=11.826)$ had WMI scores comparable to those in the WAIS standardization sample $(\mathrm{M}=100, \mathrm{SD}=15)$.

The $t$-test analysis conducted on the PSI did not yield a significant group difference between college students with ADHD and the WAIS standardization sample; $t$ $(66)=-2.268, p=.027$, thus supporting the null hypothesis of no difference. Students with ADHD (M=96.78, $\mathrm{SD}=11.633)$ had PSI scores comparable to those demonstrated by the WAIS standardization sample $(\mathrm{M}=100, \mathrm{SD}=15)$. The PSI score for the ADHD sample was significant at an alpha of .05 , but did not retain significance when the Bonferroni correction was applied. The results were approaching significance $(\mathrm{p}=.027)$, however, suggesting a trend of ADHD students having lower PSI scores than those found in the standardization sample (See Table 4).

Research Question 2: Do students diagnosed with ADHD exhibit deficits in attention and response control as compared to the IVA standardization sample? (Measured by the IVA) Hypothesis 2: There will be no statistically significant difference in attention and response control scores (Full Attention Quotient Scale, Full Scale Response Control Quotient) obtained for the ADHD group when compared with the IVA standardization sample.

Research question two was assessed by conducting two one sample $t$-tests using the independent variable of ADHD diagnosis and the dependent variables of Full Scale Response Control Quotient (FSRCQ) and Full Scale Attention Quotient (FSAQ) scores. The $t$-tests were conducted as two-tailed tests with an alpha level of .05 , with a Bonferroni correction $(.05 / 2=.025)$ for multiple $t$-tests. The $t$-test analysis conducted on the FSRCQ yielded a significant group difference between college students with ADHD 
and the IVA standardization sample; $t(69)=-10.821, \mathrm{p}<.000$, thus rejecting the null hypothesis of no difference. Students with ADHD (M=68.41, SD=24.42) had significantly lower FSRCQ scores than did the IVA standardization sample (M=100, $\mathrm{SD}=15)$. A Cohen's d of 0.9929 was calculated indicating a large effect size. Approximately $44 \%$ of the variability in FSRCQ scores was accounted for.

The $t$-test analysis conducted on the FSAQ yielded a significant group difference between college students with ADHD and the IVA standardization sample; $t(69)=-$ 10.752, $\mathrm{p}<.000$, thus rejecting the null hypothesis of no difference. Students with ADHD $(\mathrm{M}=57.70, \mathrm{SD}=32.92)$ had significantly lower FSAQ scores than did the IVA standardization sample $(\mathrm{M}=100, \mathrm{SD}=15)$. A Cohen's d of 1.6537 was calculated indicating a large effect size. Approximately $64 \%$ of the variability in FSAQ scores was accounted for (See Table 5).

Research Question 3: Do students diagnosed with ADHD exhibit deficits in cognitive functioning as compared to students diagnosed with ADHD and a comorbid LD? (Measured by the WAIS-III) Hypothesis 3: There will be no statistically significant difference in WAIS-III IQ index scores (VCI, POI, WMI, and PSI) obtained for students diagnosed with ADHD as compared to students diagnosed with ADHD and a comorbid LD.

In assessing research question three, the Box's Test of Equality of Covariance Matrices was not significant and the four dependent variables were not significantly skewed. Research question three was then assessed by a one-way multivariate analysis of variance to examine the cognitive functioning of students with ADHD as compared to students diagnosed with ADHD and a comorbid LD (ADHD/LD) by using a linear 
combination of the dependent WAIS-III IQ index score variables. The null hypothesis was supported as no significant difference was found. Analysis of the data using one-way MANOVA returned a Wilk's Lambda value of .976, F of .557, and a significance level of .694 (See Table 6).

Research Question 4: Do students diagnosed with ADHD exhibit deficits in attention and response control as compared to students diagnosed with ADHD and a comorbid LD? (Measured by the IVA) Hypothesis 4: There will be no statistically significant difference in IVA attention and response control scores (Full Scale Attention Quotient, Full Scale Response Control Quotient) obtained for students diagnosed with ADHD as compared to students diagnosed with ADHD and a comorbid LD.

In assessing research question four, the Box's Test of Equality of Covariance Matrices was not significant and the two dependent variables were not significantly skewed. Research question four was then assessed by a one-way multivariate analysis of variance to examine the attention and response control skills of students with ADHD as compared to students diagnosed with ADHD and a comorbid LD (ADHD/LD) by using a linear combination of the dependent IVA index score variables. The null hypothesis was supported as no significant difference was found. Analysis of the data using one-way MANOVA returned a Wilk's Lambda value of .993, F of .346, and a significance level of .708 (See Table 7).

Research Question 5: Do students diagnosed with ADHD exhibit significant and unusual discrepancies between their index scores (VCI/POI, VCI/WMI, VCI/PSI, POI/WMI, POI/PSI, WMI/PSI) as compared to the estimated base rates of such differences occurring in the WAIS-III standardization sample? (Measured by the WAIS- 
III) Hypothesis 5: Students diagnosed with ADHD will not exhibit significant and unusual discrepancies between their index scores (VCI/POI, VCI/WMI, VCI/PSI, POI/WMI, POI/PSI, WMI/PSI) as compared to the estimated base rates of such differences occurring in the WAIS-III standardization sample.

The fifth research question was assessed by calculating the differences between the WAIS-III index scores (VCI/POI, VCI/WMI, VCI/PSI, POI/WMI, POI/PSI, WMI/PSI) for the ADHD sample. Next, the differences were examined for statistical significance at the $\mathrm{p}<.05$ level according to the appropriate age specific tables found in Appendix B, Table B.1 of the WAIS-III Administration and Scoring Manual. Given that a statistically significant difference in index scores may not represent a clinically significant difference, the frequency of occurrence in the standardization sample according to tables found in Sattler (2001) Appendix C, Tables C-4 and C-5 were also considered. Sattler (2001) suggests that differences between scores that occur in less than $15 \%$ of the standardization sample should be judged as unusual. A cut off score of less than $15 \%$ was thus used in the calculation of percentages for the study sample.

The null hypothesis that there would be no discrepancies between WAIS-III index scores for students diagnosed with ADHD was rejected. Of the 70 students with ADHD, approximately half had significant discrepancies between all of their index scores. Statistically significant discrepancies ranged from $44.29 \%$ for POI/WMI and WMI/PSI to $55.71 \%$ for POI/PSI. Further, approximately $30 \%$ of the sample had unusual discrepancies or discrepancies that occurred in less than $15 \%$ of the standardization sample. The percentage of unusual discrepancies ranged from $30 \%$ for POI/WMI to $52.86 \%$ for POI/PSI (See Table 8). The greatest level of discrepancy occurred between 
the Perceptual Organization Index (POI) and Processing Speed Index (PSI) scores, with $53 \%$ of the students with ADHD demonstrating unusual discrepancies.

Research Question 6: Do students diagnosed with ADHD exhibit deficits in executive functioning ( $\geq 1$ SD below standardization sample mean)? (Measured by the D-KEFS) Hypothesis 6a: Students diagnosed with ADHD will not exhibit deficits in cognitive flexibility as measured by the following D-KEFS scores: Trail Making Test (TMT) Number-Letter Switching Completion Time, Verbal Fluency Category Switching Total Correct Responses, Design Fluency Total Correct Switching, and Color-Word Interference Test Inhibition/Switching Completion Time. Hypothesis 6b: Students diagnosed with ADHD will not exhibit deficits in verbal fluency as measured by the following D-KEFS scores: Verbal Fluency Letter Fluency Total Correct, and Verbal Fluency Category Fluency Total Correct. Hypothesis $6 c$ : Students diagnosed with ADHD will not exhibit deficits in non-verbal fluency, as measured by the following DKEFS scores: Design Fluency Total Correct Filled Dots, and Design Fluency Total Correct Empty Dots. Hypothesis 6d: Students diagnosed with ADHD will not exhibit deficits in concept formation as measured by the following D-KEFS scores: Sorting Test Free Sorting Confirmed Correct Sorts, Sorting Test Free Sorting Description Score, Sorting Test Sort Recognition Description Score, Twenty Questions Test Initial Abstraction Score, Proverb Test Free Inquiry Total Achievement Score, and Word Context Total Consecutively Correct. Hypothesis 6e: Students diagnosed with ADHD will not exhibit deficits in problem solving as measured by the D-KEFS Twenty Questions Test Total Weighted Achievement score. Hypothesis 6f: Students diagnosed 
with ADHD will not exhibit deficits in planning as measured by the D-KEFS Tower Test Total Achievement Score.

Research question six was assessed with descriptive statistics. The percentage of students exhibiting executive functioning deficits (greater than or equal to one standard deviation below the standardization sample mean) in the domains of cognitive flexibility, concept formation, verbal and non-verbal fluency, planning, and problem solving are presented. Deficits in executive functioning were determined based on an idiographic review of each student's scores. For D-KEFS tests that provide contrast scores (Trail Making Test, Verbal Fluency, Design Fluency, Color-Word Interference, and Sorting Test), a deficit in executive functioning was substantiated only if the contrast scores indicate a deficit in executive functioning over and above deficits in the fundamental skills measured by the test.

In assessing research question six, it appeared that college students with ADHD did not consistently exhibit deficits in a particular domain of executive functioning, thus supporting the null hypothesis of no difference. Percentages of students exhibiting executive functioning deficits in the various domains measured ranged from $1 \%$ to $20 \%$. Percentages of students exhibiting executive functioning deficits in various domains measured were as follows: cognitive flexibility ( $7 \%$ to $11 \%$ ), verbal fluency (11\% to $20 \%$ ), design fluency (9\%), concept formation (1\% to $17 \%$ ), problem solving (4\%), and planning (13\%).

Scores that allow for differentiation of the component skills necessary for task completion were primarily presented for the executive functioning domain of cognitive flexibility. Before taking component functions into consideration, deficits in cognitive 
flexibility appeared more prominent, with percentages of students exhibiting deficits ranging from $9 \%$ to $26 \%$ (as compared to $7 \%$ to $12 \%$ ) (See Table 9).

Additional analyses. Also interesting is that strengths in various domains of executive functioning tasks appear more prominent then deficits. Percentages of students exhibiting executive functioning strengths in the various domains measured are as follows: cognitive flexibility ( $0 \%$ to $11 \%$ ), verbal fluency (32\% to $36 \%$ ), design fluency (14 to $24 \%$ ), concept formation (17\% to $27 \%$ ), problem solving (41\%), and planning (17\%). Before component functions were taken into consideration, strengths in cognitive flexibility also appeared more prominent, with percentages of students exhibiting strengths ranging from $3 \%$ to $30 \%$ (as compared to $0 \%$ to $11 \%$ ) (See Table 10 ).

Interestingly, 74\% $(\mathrm{n}=52)$ of the students with ADHD exhibited one or more scores that were significantly below average. All 70 students with ADHD had at least one score, and 66 students had at least 2 scores that were one or more standard deviations above or below the standardization sample mean. Further, $46(65.71 \%)$ of the 70 students with ADHD had at least one score that was significantly below average, and at least one score that was significantly above average.

Although the hypotheses did not address means for measures of executive functioning, they are reported as supplemental information in Table 11. The mean scores all fell within the average range and ranged from 9.2 to 11.35 for executive functioning domains and from 8.8 to 10 for non-executive functioning domains. Examination of means alone may lead to an assumption that the executive functioning skills among college students with ADHD are average. An idiographic review of each student's scores, 
however, indicates variability in executive functioning performance inclusive of average, significantly below average, and significantly above average scores.

Based on the finding that college students with ADHD performed poorly on response inhibition as measured by the IVA, a condition on the D-KEFS that also assesses inhibition was reviewed. The percentage of students with ADHD who exhibited deficits in response inhibition as measured by the D-KEFS Color Word Inhibition task was $21.43 \%$. This means that a higher percentage of students with ADHD exhibited a deficit in response inhibition than any other domain of executive functioning. Further, only $4.29 \%$ of students exhibited a strength in response inhibition.

\section{Summary of Results}

The results of this study offer some insight into neuropsychological functioning of college students with ADHD. This research study found that students with ADHD had significantly higher VCI and POI scores, but comparable WMI and PSI scores as compared to the WAIS-III standardization sample. Though not statistically significant with the Bonferroni correction, there was a trend for students with ADHD to have lower PSI scores as compared to the standardization sample.

Performance on sustained attention and response inhibition, as measured by the IVA, was significantly lower for the ADHD sample when compared with the general population. Students with ADHD exhibited extremely impaired abilities to sustain attention and inhibit responses. Differences were not found between college students with ADHD and college students with ADHD and a comorbid LD. Approximately half of the students with ADHD had significant discrepancies between all of their index scores, and approximately $30 \%$ of the sample had unusual discrepancies. The largest discrepancy 
occurred between POI and PSI, with 53\% of the students with ADHD demonstrating this unusual discrepancy.

Students in this sample did not appear to exhibit deficits in a particular domain of executive functioning. Percentages of students exhibiting executive functioning deficits in various domains ranged from a minimum of $1 \%$ to a maximum of $20 \%$. An additional review of the D-KEFS data revealed that approximately one-fifth of the students with ADHD also exhibited deficits in response inhibition as measured by the Color Word Inhibition Test.

Interestingly, strengths in various domains of executive functioning were more prominent than deficits, with percentages of students exhibiting strengths ranging from $0 \%$ to $41 \%$. An additional observation is that the majority of students $(94 \%)$ had at least 2 scores that were one or more standard deviations above or below the standardization sample mean. More than half of the students $(65.71 \%)$ had at least one score that was significantly below average, and at least one score that was significantly above average. Additional descriptive analyses of the D-KEFS tests revealed that the mean scores for all executive functioning tests fell in the Average range. An idiographic review of each student's scores, however, indicated variability in executive functioning performance inclusive of average, significantly below average, and significantly above average scores. 


\section{CHAPTER 4}

\section{Discussion}

The primary objective of this study was to examine the neuropsychological functioning of college students with Attention Deficit Hyperactivity Disorder (ADHD). Specifically, we examined performance on measures of cognition, sustained attention, response inhibition, and executive functioning. This chapter provides a brief overview of the literature, purpose of the study and methodology, as well as discussion of the findings, limitations, clinical implications, and directions for future research.

Individuals diagnosed with ADHD often experience lifelong impairment in several functional domains (Mick, Faraone, \& Biederman, 2004; Mick, Faraone, Biederman, \& Spencer, 2004; Murphy, Barkley, \& Bush, 2002; Torgersen, Gjervan, \& Rasmussen, 2006). Although there is no definitive test for identifying ADHD, neuropsychological tests have been proposed as one means of enhancing identification of ADHD in adults. Researchers have theorized that deficits in executive functioning underlie the symptom appearance and resulting impairment experienced by individuals with ADHD (Barkley, 2006). A decade of research addressing the relationship between executive functioning and ADHD in adults suggests that although adults with ADHD exhibit diffuse executive functioning deficits, these deficits alone have not accounted for the full symptom appearance of ADHD (Hervey, Epstein, \& Curry, 2004; Schoechlin \& Engel, 2005). Also, many of the executive functioning assessments used to date have relatively poor reliability and validity, and do not differentiate between the basic skills required for task completion and actual executive functioning skills (Lezak, 2002; Willcutt, Doyle, Nigg, Faraone, \& Pennington, 2005). This differentiation is particularly 
important in light of meta-analytic findings which suggest that fundamental skills provide nearly equal discriminatory validity for differentiating adults with and without ADHD (Boonstra et al., 2005).

Unfortunately, individuals with ADHD are less likely to enter college and are even less likely to successfully complete a college education than are their non-ADHD counterparts (Barkley, 2002). Research has suggested that college students with ADHD may differ from individuals with ADHD in the general population in level of education, degree of impairment, and cognitive abilities (Biederman, 1993). The development of a range of compensatory skills to adapt to academic demands has also been hypothesized as a contributing factor to the success of children and adolescents with ADHD (Heiligenstein, 1996; Ramsay \& Rostain, 2006; Richard, 1995; Weyandt, 2003). However, these compensatory skills may not be as effective for the challenging demands of postsecondary education and college life (Shaw-Zirt, Popali-Lehane, Chaplin, \& Bergman, 2005).

The increasing proportion of students with ADHD who are pursuing postsecondary education and the well-documented academic and psychosocial impairment among individuals with ADHD brings to light the critical importance of better understanding this disorder among college students (Biederman et al., 2006; Brod, Perwien, Adler, Spencer, \& Johnston, 2005; Heiligenstein, Guenther, Levy, Savino, \& Fulwiler, 1999; Murphy, Barkley, \& Bush, 2002). Better understanding the neuropsychological strengths and weaknesses of college students with ADHD may improve treatment planning and enable us to provide these students with support not only for academic success, but also for an improved quality of life. 
Research regarding the neuropsychological performance of college students to date appears to suggest that college students with ADHD are less impaired than their counterparts in the general population, and may only be differentiated by a measure of continuous performance (Weyandt, Linterman, \& Rice, 1995; Weyandt, Rice, \& Linterman, 1998; Weyandt, Mitzlaff, \& Thomas, 2002; Advocat, Martino, Hill, \& Gouvier, 2007). This suggestion is based, however, on only a few studies with limited samples, power and generalizability, and clearly implicates the need for additional research addressing the neuropsychological performance of college students with ADHD.

Therefore, the purpose of this study was to examine neuropsychological functioning among college students with ADHD, using a comprehensive battery of neuropsychological measures with well established psychometric properties, to determine if it is possible to obtain a picture of the neuropsychological profile exhibited by college students with ADHD. Study participants included college students diagnosed with ADHD or ADHD/LD following ADHD/LD assessment at a large university in the midAtlantic region between August of 2003 and March of 2008. Students with comorbidity other than a learning disability were excluded from the study.

Assessment batteries were administered by one of nineteen psychology interns and licensed psychologists, over the course of five years. Testing for ADHD required approximately five hours, and testing for ADHD/LD required approximately eight hours. Students being treated with stimulant medication were required to abstain from medication use on the day of testing. Assessment instruments used in determining diagnosis of ADHD or LD include: Assessment Screening Questionnaire, ADHD/LD Testing Background Information Form, College ADHD Response Evaluation - Student 
Response Inventory, College ADHD Response Evaluation - Parent Response Inventory, Woodcock-Johnson - Third Edition, Wechsler Adult Intelligence Scale - Third Edition (WAIS-III), Integrated Visual \& Auditory Continuous Performance Test (IVA), DelisKaplan Executive Function System (D-KEFS), and an informal clinical interview.

The following research questions were examined: 1) Do students diagnosed with ADHD exhibit cognitive deficits as compared to the WAIS-III standardization sample; 2) Do students diagnosed with ADHD exhibit deficits in attention and response control as compared to the IVA standardization sample; 3) Do students diagnosed with ADHD exhibit deficits in cognitive functioning as compared to students diagnosed with ADHD and a comorbid LD; 4) Do students diagnosed with ADHD exhibit deficits in attention and response control as compared to students diagnosed with ADHD and a comorbid LD; 5) Do students diagnosed with ADHD exhibit significant and unusual discrepancies between their WAIS-III index scores as compared to the estimated base rates of such differences occurring in the WAIS-III standardization sample; and 6) Do students diagnosed with ADHD exhibit deficits in executive functioning as measured by the DKEFS?

One sample t-tests with Bonferroni correction were used to assess the cognitive, attentional and response inhibition skills among adults with ADHD. Multivariate analysis of variance was used to compare the WAIS-III index scores and the IVA global scores for students diagnosed with ADHD as compared to students diagnosed with ADHD and a comorbid LD. Descriptive statistics were used to examine discrepancies between WAISIII index scores as well as to explore the executive functioning performance of college students with ADHD. 
The resulting analysis showed us the following: 1) Students with ADHD had significantly higher VCI and POI scores, but comparable WMI and PSI scores as compared to the WAIS-III standardization sample; 2) Students with ADHD exhibited extremely impaired abilities to sustain attention and inhibit response as compared with the IVA standardization sample; 3) Differences in cognitive, attentional, and response inhibition skills were not found between college students with ADHD and college students with ADHD and a comorbid LD; 4) Approximately half of the students with ADHD had significant discrepancies between all of their index scores, and approximately $30 \%$ of the sample had unusual discrepancies, with $53 \%$ of the students demonstrating unusual discrepancies between POI and PSI; and 5) Students in this sample did not appear to exhibit deficits in a particular domain of executive functioning. Supplemental analyses of the D-KEFS data revealed that strengths in various domains of executive functioning were as common as deficits.

Demographic Characteristics

The majority of students in the ADHD and ADHD/Learning Disordered (ADHD/LD) groups were undergraduates between the ages of 18 and 21, thus representative of a typical college student population. The gender distribution was comparable (38 men and 32 women in the ADHD group and 18 men and 13 women in the ADHD/LD group). Equivalent gender distribution among college students with ADHD was also found by DuPaul et al. (2001). This differs from the vast literature attesting to a higher prevalence of ADHD among males than females (Barkley, 2006; Carlson \& Mann, 2000). This anomaly may reflect that the gender ratios for males and females are skewed by referral patterns as males tend to exhibit externalizing behaviors 
and are thus more likely to be referred. Additional longitudinal research is needed to better understand the nature of ADHD throughout the lifetime, including potential gender differences.

A review of subtypes represented in the participants reflects that ADHD Combined Type was the most common subtype for the ADHD group (67\%) and the ADHD/LD group (41\%). This finding is consistent with results reported in the DSM-IVTR (APA, 2000), but differs from both the DuPaul et al. (2001) study, in which ADHD Predominantly Hyperactive-Impulsive was the most prominent subtype, and the Heiligenstein, Conyers, Berns, and Smith (1998) study, in which ADHD Predominantly Inattentive Type was most prominent. This disparity may be due to sample and diagnostic assessment differences. For example, diagnosis of ADHD was substantiated by selfreport assessment instruments only in both the Heiligenstein et al. (1998) and DuPaul et al. (2001) studies. A more rigorous battery of assessment instruments as well as a clinical interview was used to determine ADHD diagnosis in this study. Further, the sample included students who sought testing for the purposes of receiving academic accommodations. It is possible that this sample differs from other students with ADHD who do not pursue academic accommodations.

The average college grade point averages (GPA) for the ADHD and ADHD/LD samples were 2.23 and 2.57, respectively. This statistic does not include $30 \%$ of the sample who were first semester freshmen yet to achieve a GPA. While many variables impact GPA, these grades may reflect a form of functional impairment.

\section{Cognitive Functioning}


The hypothesis that there would be no difference in the cognitive performance obtained for the ADHD group when compared with the WAIS-III standardization sample was partially supported. College students with ADHD in the current sample had significantly higher Verbal Comprehension Index (109) and Perceptual Organization Index (109) scores, and comparable Working Memory Index (102) and Processing Speed Index (97) scores when compared with the WAIS-III standardization sample. The difference between college students with ADHD and the WAIS-III standardization sample on the Processing Speed Index approached significance, suggesting a trend of ADHD students having lower processing speeds relative to the standardization sample. All of the scores, however, fell within the Average range.

This finding is significant when considering current research on adults with ADHD. In general, researchers have suggested that adults with ADHD have comparable (Nigg et al., 2005; Schweitzer, Hanford, \& Medoff, 2006) to lower IQ scores as compared with non-ADHD adults (Bridgett \& Walker, 2006; Hervey, Epstein, \& Curry, 2004; Murphy, Barkley, \& Bush, 2001; Murphy, 2002; Schoechlin \& Engel, 2005). Bridgett and Walker (2006) emphasized that the difference found in IQ scores in their meta-analysis was statistically significant, but not clinically meaningful.

The finding that college students with ADHD in the current sample had comparable to higher IQ scores appears to support theories suggesting that college students may represent a unique subset of individuals with ADHD who are less impaired (Biederman, 1993; Glutting, Youngstrom, \& Watkins, 2005; Heiligenstein, Conyers, Berns, \& Miller, 1998). Glutting et al. (2005), in specific, suggested that college students with ADHD are likely to have higher ability levels, better academic success in primary 
and secondary school, and greater compensatory skills than individuals with ADHD from the general population.

Only a few studies provide data regarding the intellectual functioning of college students with ADHD. Advocat, Martino, Hill, and Gouvier (2007), Weyandt, Mitzlaff, and Thomas (2002), and Shaw-Zirt, Popali-Lehane, Chaplin, and Bergman (2005) found no significant differences in the intellectual performance between college students with ADHD and control groups on the WAIS-III, WAIS-R, and Kaufman Brief Intelligence Scale, respectively. The discrepancy between the results of the current study and those found by Advocat et al. (2007), Shaw-Zirt et al. (2006), and Weyandt et al. (2002) is not surprising when considering the samples. We compared college students with ADHD to the general population while the other studies made comparisons with other college students. College students with ADHD had cognitive abilities comparable to or higher than the general population according to the current study, and cognitive abilities comparable to other college students according to other studies (Advocat et al., 2007; Shaw-Zirt et al., 2006; Weyandt et al., 2002). The results regarding the intellectual functioning of college students with ADHD in other studies should be interpreted with caution, however, given the extensive limitations of the studies used to support the results, including small sample sizes, variable diagnostic and inclusion criteria, and the impact of moderating variables. Limitations aside, the results appear to support the notion that college students with ADHD exhibit fewer cognitive deficits than adults with ADHD in the general population, as hypothesized by Heiligenstein, Conyers, Berns, and Miller (1998). 
Examination of WAIS-III index scores for the current sample in comparison to meta-analytic results also appears to lend support to the theory that college students with ADHD may represent a subset of individuals with ADHD who are less impaired. Metaanalytic findings indicated that adults with ADHD performed significantly worse than non-ADHD adults in the domain of verbal intelligence, which included the three subtests that make up the WAIS-III Verbal Comprehension Index (WAIS-R Similarities, Information, Vocabulary) plus a measure of reading (Schoechlin \& Engel, 2005). Adults with ADHD also performed significantly worse than non-ADHD adults in the domain of visual-figural problem solving (WAIS-R Block Design, Picture Completion, Picture Arrangement; Rey-Osterrieth Complex Figure; Embedded Figures Test), which included 2 of the 3 subtests that made up the WAIS-III Perceptual Organization Index. This is interesting given that students in the current sample achieved mean scores that did not reflect a deficit in performance. It is possible, however, that the results were moderated by the additional measures used to comprise the verbal intelligence and visual-figural problem solving domains.

\section{Attention and Response Inhibition}

The hypothesis that there would be no statistically significant difference in attention and response control scores obtained for the ADHD group when compared with the IVA standardization sample was strongly rejected in the results of this study. Students with ADHD had significantly lower scores in relation to the IVA standardization sample, indicating an extremely impaired ability to sustain attention and to inhibit responses. What we know about adults and college students with ADHD is that they consistently demonstrate impaired performance on measures of sustained attention and response 
inhibition as measured by tests of continuous performance in this and other studies (Boonstra, Oosterlaan, Sergeant, \& Buitelaar, 2005; Epstein, Johnson, Varia, \& Conners, 2001; Hervey, Epstein, \& Curry, 2004; Murphy, Barkley, \& Bush, 2001; Nigg et al., 2005; Schoechlin \& Engel, 2005; Walker, Shores, Troller, Lee, \& Sachdev, 2000; Weyandt, Mitzlaff, \& Thomas, 2002). These results suggest that a measure of attention and response inhibition plays a vital role in the diagnosis of ADHD and understanding its impairment.

\section{ADHD and Learning Disorders}

Results from this study supported the hypothesis that performance on cognition, attention, and response control did not differ for college students with ADHD as compared to college students with ADHD and a comorbid Learning Disorder (LD). In fact, the means for the groups across measures of intellectual functioning, attention, and response inhibition were nearly exact. It remains unknown the nature of the functional impact of learning disorders that occur either alone or comorbidly with ADHD. Additional research including a "pure" LD sample would shed light on this important question. Unfortunately, the small sample size for the ADHD/LD group ( $\mathrm{n}=31)$ did not provide adequate power to detect a difference. While nearly equivalent performance appears to suggest this would not have much impact, additional research with adequate sample sizes are needed to statistically support or negate this finding.

\section{WAIS-III Index Discrepancy Scores}

Results from the current study do not appear to support the hypothesis that students diagnosed with ADHD would not exhibit significant and unusual discrepancies between their WAIS-III index scores as compared to the estimated base rates of such 
differences occurring in the WAIS-III standardization sample. Instead, the results found that approximately half of the students had significant discrepancies between all of their WAIS-III index scores, with discrepancies ranging from $44 \%$ to $56 \%$. Further, approximately $30 \%$ to $53 \%$ of the students had unusual discrepancies. The largest unusual discrepancy occurred between the Perceptual Organization Index and Processing Speed Index scores. Fifty-three percent of the students with ADHD demonstrated this rare discrepancy. This suggests that the nonverbal reasoning, spatial processing skills, attentiveness to detail and visual-motor integration skills of college students with ADHD are unusually discrepant from their ability to process simple or routine visual information quickly and efficiently and to quickly perform tasks based on that information. It is important to note, however, that the student's Perceptual Organization (109) and Processing Speed (97) Index scores both fell in the Average range. The discrepancies between an individual's scores may be clinically meaningful in better understanding the neuropsychological functioning of college students with ADHD and warrants further research. Woods, Lovejoy, and Ball (2002) also found index score discrepancies.

\section{Executive Functions}

Our results support the hypothesis that students diagnosed with ADHD would not exhibit deficits in the executive functioning domains of cognitive flexibility, verbal fluency, non-verbal fluency, concept formation, problem solving, and planning. An idiographic review of each student's scores, taking the role of component scores into consideration, appears to suggest that college students with ADHD do not consistently exhibit deficits in a particular domain of executive functioning. Percentages of students 
exhibiting executive functioning deficits in the various domains measured ranged from $1 \%$ to $20 \%$.

Our finding that executive functions were not generally impaired in college students with ADHD is consistent with meta-analytic review of the neuropsychological functioning of adults with ADHD. Schoechlin and Engel (2005) discuss the surprisingly small effect sizes found for the executive functioning domain in light of Barkley's theory suggesting that deficits in inhibition lead to secondary deficits in executive functions. They believe that this discrepancy may reflect frontal lobe maturation or population bias, given that most adults in the meta-analytic study were intelligent without comorbid conditions.

Boonstra et al. (2005) and Hervey et al. (2004) also found that neuropsychological deficits among adults with ADHD were diffuse among both executive functioning and non-executive functioning domains. The authors note that the presence of deficits in nonexecutive functioning tasks suggests that impairment in executive functioning only partially characterizes the impairments among adults with ADHD. Results from the current study appear consistent with those found by meta-analytic review. Though diffuse impairments were found in each domain, a domain specific deficit was not identified.

During a secondary analysis of the D-KEFS data, we found that $21 \%$ of the students with ADHD also exhibit deficits in response inhibition as measured by the Color Word Inhibition task. Difficulty with response inhibition as evidenced by both the DKEFS and the IVA provides substantiation of the importance of response inhibition by two separate assessment instruments. These results indicate that response inhibition is a 
domain of particular importance for college students with ADHD and warrants further research.

There may also be a need for additional research addressing variability in attention over time, a construct that is difficult to capture. All 70 (100\%) students with ADHD had at least one score that was one or more standard deviations above or below the standardization sample mean, and $94 \%$ of the students had at least two of these scores. Three-fourths of the students also had one or more scores that were significantly below average. Further, $66 \%$ of the students had at least one score that was significantly below average, and at least one score that was significantly above average.

While these scores likely represent true areas of strength and weakness, they may also reflect the variability in performance that is inherent with ADHD. If executive functioning were viewed as a unitary construct captured by all nine D-KEFS tests of executive functioning, then variability in performance across several tests would lend itself to an interpretation of variability in performance over time. However, executive functioning is rarely viewed as a unitary construct. The D-KEFS provides an objective measure of variability in performance over time for only one of the nine tests, Verbal Fluency. Research including a measure of performance variability may prove useful in more clearly detailing the nature of ADHD, a recommendation supported by Boonstra, Oosterlaan, Sergeant, and Buitelaar (2005).

An important contribution of this study is the finding that measures of executive functioning that do not take component scores into consideration may result in overestimation of deficits. The percentages of students exhibiting deficits in the domain of cognitive flexibility decreased in as little as $2 \%$ for the Trail Making Test to a high of 
$14 \%$ for the Color Word Interference Test. For example, deficits on the Color Word Inhibition/Switching task dropped from $26 \%$ of the sample to only $12 \%$ of the sample when taking performance on component skills into consideration. This means that $14 \%$ of the students with ADHD would have been incorrectly identified as having a deficit in cognitive flexibility. Instead, these students may have exhibited a deficit in their ability to quickly read the names of colors, or to quickly identify and verbalize the name of an ink blot color. Researchers emphasize the importance of differentiating between the fundamental skills required for task completion and the executive functioning skills purportedly measured by assessment instruments (Boonstra et al., 2005; Willcutt, Doyle, Nigg, Faraone, \& Pennington, 2005). This differentiation is particularly important in light of meta-analytic findings which have suggested that component skills provide nearly equal discriminatory validity for differentiating adults with and without ADHD (Boonstra et al., 2005).

Also of importance is the observation that strengths in various domains of executive functioning appear more prominent than deficits. Percentages of students exhibiting executive functioning strengths in the various domains measured ranged from $0 \%$ to $11 \%$ for measures of cognitive flexibility to $41 \%$ for problem solving. Very few studies emphasize strengths in executive functioning. However, identification of these strengths is essential for treatment planning as a means of adaptive compensation for areas of weakness.

The development of a range of compensatory skills to adapt to academic demands has also been hypothesized as a contributing factor to the success of students with ADHD (Heiligenstein, 1996; Ramsay \& Rostain, 2006; Richard, 1995; Weyandt, 2003). The use 
of compensatory strategies may have masked functional impairments in the current sample. In addition, the role of higher intellectual functioning may have impacted performance on other measures. A topic of significant debate is the role of intelligence in executive functioning (Bridgett \& Walker, 2006). Hervey, Epstein, and Curry (2006, p. 498) argue that "removing variability associated with differential IQ performance may be removing part of what constitutes ADHD." It is recommended that results be presented both with and without controlling for or covarying IQ, given that no consensus has been reached regarding this question (Bridget \& Walker, 2006; Murphy, Barkley, \& Bush, 2001). The role of IQ was not assessed because measures of central tendency were not included in the hypotheses in this study. It is recommended that future studies report results with and without IQ covaried, until there is agreement among researchers.

We used nine tests to represent executive functioning instead of the more common approach of only using a single test (i.e. Trail Making Test or Wisconsin Card Sorting Test). Our results assert the importance of using a battery of assessments to capture the nature of neuropsychological functioning, which was also suggested by Schoechlin and Engel (2005). The use of a battery of neuropsychological measures appears imperative until the meaning of executive functioning is clarified, and until measures that clearly differentiate individuals with ADHD from those without ADHD are determined. Instruments detailing the specific neuropsychological strengths and weaknesses, particularly for individuals pursuing secondary education are also warranted.

\section{Limitations}

A significant limitation of this study is generalizability. Results from the current study represent a convenience sample consisting of only students at a large university in 
the mid-Atlantic region who received ADHD/LD assessment for the purposes of receiving academic accommodations. Thus, the results may not be generalizable to students with ADHD from other regions or students who do not pursue academic accommodations. Ethnic diversity was also virtually absent in this study, although comparable to the diversity represented at this university. Further, the current study used pre-existing data which did not allow for manipulation of sample sizes. While adequate sample sizes were available for students with ADHD, the sample size of students with ADHD/LD was not sufficient, resulting in decreased power for detecting significant differences.

Another limitation of this study involves the methodology. A causal comparative study contains the weakness associated with lack of randomization. It is possible that the groups may be different on other important variables in addition to the independent variable of diagnosis (ADHD, ADHD/LD). This results in difficulty in establishing a firm cause-effect relationship.

Additional limitations relate to the protocol for obtaining data. The assessment batteries were administered by one of nineteen psychology pre-doctoral interns and licensed psychologists, over the course of five years. Consistency in adherence to standardization cannot be assured, though each battery was reviewed and signed by one of two Assessment Coordinators. In addition, a formal clinical interview was not used, though potential comorbid conditions were fully explored.

The generalization of results from ADHD testing to real-world learning environments has also been called into question. Testing is typically completed on a oneon-one basis in an environment with minimal distractions. Several tests, particularly tests 
of executive functioning, also tend to be highly engaging. These factors could potentially improve testing performance, thus obscuring areas of impairment that are typically present in general education settings. Research seeking to identify the impact of neuropsychological impairments on everyday academic and social functioning holds great clinical implications.

\section{Implications for Intervention}

This study provides insight into the neuropsychological functioning of college students with ADHD. Several of the findings provide useful clinical implications regarding treatment planning for college students with ADHD. In addition, results of this study provide insight into important directions for future research regarding college students with ADHD.

A large emphasis in the ADHD literature has addressed the role of executive functioning. Results from the current sample suggest that on average, college students with ADHD do not consistently exhibit deficits in a particular domain of executive functioning, though individually most students exhibit diffuse deficits. Deficits in sustained attention and response inhibition, however, were prominent in this group of students. Additional research regarding the functional implications of these deficits for the academic performance of college students with ADHD may be clinically useful.

The results of the current study also highlight the importance of using a battery of measures to assess for the neuropsychological functioning of college students with ADHD. This battery of assessment instruments should include measures of the domains implicated in ADHD (i.e. attention, response inhibition, executive functioning) as well as measures with solid psychometric properties, measures that provide for differentiation of 
component versus executive functioning skills, and measures that provide information regarding variability in performance.

The results of this study along with established recommendations for the treatment of ADHD implicate the use of a multimodal approach including medication, academic accommodations, and psychotherapeutic interventions (Asherson, 2005). Stimulant medication is considered the treatment of choice for ADHD in both children and adults (Asherson, 2005; Faraone, Spencer, Aleardi, Pagano, \& Biederman, 2004; Fitzgerald, 2001; Weiss, Murray, \& Weiss, 2002). In addition to symptom reduction, adults with ADHD have demonstrated improvements in response inhibition (Schweitzer et al., 2004), and working memory, sustained attention, and motor and processing speed following stimulant medication use (Turner, Blackwell, Dowson, McLean, \& Sahakian, 2005). The finding that stimulant medication improved response inhibition, sustained attention, and processing speed for adults with ADHD suggests that stimulant medication may also be particularly helpful in decreasing functional impairment for college students with ADHD.

Medication does not inherently provide individuals with coping skills and strategies for managing functional or quality of life impairments associated with ADHD (Safren, Sprich, Chulvick, \& Otto, 2004). Many students with ADHD may experience significant academic problems for the first time upon arriving to college as their previous coping mechanisms may not continue to be as effective for the challenging demands of college life (Shaw-Zirt, Popali-Lehane, Chaplin, \& Bergman, 2005). These difficulties include a transition to advanced education, which requires sustained attention and inhibition (Weyandt, 2003). At the postsecondary level, students are now expected to 
take responsibility for planning, structuring their time, and securing their medical and academic accommodations that were previously taken care of by others (Ramsay \& Rostain, 2006). Deficits in attention and inhibition are likely to become more apparent and problematic in the absence of external supervision. Postsecondary support systems including academic, counseling, disability, and housing services can be particularly helpful during this transition.

Overall, it appears that the intellectual level and executive functioning skills of college students with ADHD are average. However, students with ADHD are extremely impaired on a relatively simple task of sustained attention and response inhibition. Thus, the implementation of academic accommodations that specifically target deficits with sustained attention and response inhibition is a logical next step.

Academic interventions such as peer tutoring, note taking skills, study and organizational skills, instructional and task modifications (i.e. assignment choices, oral presentation, explicit instructions, computer-assisted instruction), self-monitoring and reinforcement, and homework interventions including goal setting and external structuring have demonstrated some success in improving the functional impairment of children and adolescents with ADHD (Raggi \& Chronis, 2006). However, literature regarding the efficacy of academic accommodations for college students with ADHD is largely absent. Although academic accommodations for college students with ADHD make intuitive sense, most lack empirical studies investigating their effectiveness (Weyandt \& DuPaul, 2006). Nonetheless, the following recommendations intuitively follow the findings that the abilities to sustain attention and to inhibit responses were extremely impaired in college students with ADHD. 
The first recommendation is the allotment of additional time to complete examinations. This additional time may provide students with ADHD with the opportunity to "attend" an amount of time commensurate with other college students who do not have difficulty with attention. Additional time also appears relevant when taking into consideration the trend of lower processing speed scores when compared with the general population. Similarly, the provision of lecture notes may provide written structure for attending in class. The provision of notes would also provide students with a more organized and complete set of notes that would otherwise be difficult to obtain by a student with an extremely impaired ability to attend and to inhibit off-task behaviors. An alternative option would be audio recording lectures, which could be reviewed for consistency with the notes taken. Tutoring may also provide the opportunity to clarify and better integrate the information presented in class and through reading, particularly for material that may have been missed due to distractibility or difficulty attending.

Additional recommendations for academic accommodations relate to the learning and testing environment. The presentation of information in both visual and auditory modalities may improve attention and decrease distractibility. In addition, the use of a computer for completing essay tests may provide students with spell check and the ability to better organize their thoughts. Sitting in the front of the classroom, and completing testing in an environment with minimal distractions may also improve attention and decrease distractions. Though not an academic accommodation, the use of a daily planner is also recommended. An engaging electronic device that is easily accessible may be particularly helpful. 
Further, an ideographic review of each student's neuropsychological strengths and weaknesses is recommended as the identification of these strengths and weaknesses may be particularly useful for college students seeking academic accommodations. An emphasis on strengths, in particular, may not only enhance learning and performance, but also provide a sense of accomplishment and faith in one's ability to succeed. Ramsay and Rostain (2006) also emphasize the important contribution that neuropsychological testing can provide in guiding the development of treatment plans tailored to each student's relative strengths and weaknesses. To date, research investigating the efficacy of interventions tailored specifically to an individual's strengths and weaknesses as identified by neuropsychological testing is not available. Future research in this area may prove particularly useful for college students with ADHD.

Research seeking to clarify whether students with ADHD develop compensatory strategies and how these strategies impact adaptive functioning is also important as compensatory strategies could be taught in conjunction with neuropsychological strengths. Together, individual strengths and adaptive compensatory strategies may hold great clinical implications for the academic success of students with ADHD. Research addressing the efficacy of academic accommodations for ADHD is also needed.

Therapeutic interventions are also recommended in consideration of existing guidelines for the treatment of ADHD (Asherson, 2005), though the results of this study do not directly implicate therapy. Therapeutic interventions such as psychotherapy, coaching, social skills training, support groups, and group therapy have demonstrated some efficacy in improving functioning among adults with ADHD (Asherson, 2005; Safren, 2006; Weiss, Murray, \& Weiss, 2002). Shaw-Zirt, Popali-Lehane, Chaplin, and 
Bergman (2005) also found that self-esteem and social skills may partially mediate adjustment to college. Thus, interventions to improve self-esteem and social skills may be an important avenue for bolstering students' adjustment to and success with college. Weiss et al. (2002) emphasize that psychotherapeutic interventions can aid in teaching specific strategies to improve daily functioning, in modifying beliefs about the meaning of ADHD symptoms, and in addressing any problems that may have developed as a result of ADHD. A Cognitive Behavioral Treatment approach specific to ADHD in adulthood has also been developed and found to successfully decrease ADHD symptoms, as well as symptoms of anxiety and depression (Safren, 2006).

\section{Conclusions}

The results of this study are similar to those found by the minimal other studies of college students with ADHD. It appears that a measure of inattention and response inhibition consistently differentiated students with ADHD from college students without ADHD and standardization samples. Although individuals with ADHD tend to exhibit IQs that are lower than those found in the general population, this finding does not hold true for college students. The Full Scale IQ of college students with ADHD in the current study fell at the high end of the average range. Measures of verbal comprehension and perceptual organization were also higher, whereas measures of working memory and processing speed were commensurate with the general population, with a trend for processing speed skills to fall lower than those in the standardization sample. In regards to executive functioning, results suggest that most students with ADHD tend to exhibit performance in the average range. Individually, however, most students exhibited both 
strengths and impairment in at least one domain of executive functioning. The results do not indicate a domain specific neuropsychological impairment.

Research on children with ADHD appears to support the theory that deficits in attention and response inhibition contribute to executive functioning deficits (Barkley, 2006). However, this theory does not appear to hold true for college students with ADHD. Though students with ADHD in the current sample clearly exhibited impaired attention and response inhibition skills, these deficits did not consistently translate into deficits in particular domains of executive functioning. This discrepancy may reflect frontal lobe maturation as suggested by Schoechlin and Engel (2005) or perhaps the development of adaptive compensatory skills that contributed to improved neuropsychological performance among college students with ADHD (Heiligenstein, 1996; Ramsay \& Rostain, 2006; Richard, 1995; Weyandt, 2003).

Results from the current study suggest interventions for improving the academic success of college students with ADHD. Medication is recommended as research has demonstrated that stimulant medication improves response inhibition (Schweitzer et al., 2004), working memory, sustained attention, and motor and processing speed (Turner, Blackwell, Dowson, McLean, \& Sahakian, 2005). Academic and psychological support services are also recommended. These services are particularly important as students transition to the challenging demands of college life (Shaw-Zirt, Popali-Lehane, Chaplin, \& Bergman, 2005).

Academic accommodations that specifically target deficits with sustained attention and response inhibition are also recommended. These include additional time to complete examinations, lecture notes, lecture audio-taping, tutoring, the use of a 
computer for essay exams, preferential seating, and the use of a daily planner. Further, a review of neuropsychological performance can guide the development of treatment plans tailored to each student's relative strengths and weaknesses. Therapeutic interventions such as Safren's (2006) Cognitive Behavioral Treatment approach, or interventions aimed at improving self-esteem and social skills are also recommended.

Results of this study highlight the need for further research in the field of ADHD, particularly college students with ADHD. Several researchers have suggested that inconsistent neurological profiles among individuals with ADHD may be due to deficits specific to ADHD subtype (Milich et al., 2001). In particular, research addressing sluggish cognitive tempo (SCT) is warranted as SCT may represent a distinct and unrelated disorder (Barkley, 2006; Milich, Balentine, \& Lynam, 2001). Thus, neuropsychological functioning among the different subtypes of ADHD is an important area for future research.

In addition, results of this study need to be replicated with a larger sample to more clearly determine the neuropsychological functioning of college students with ADHD, including students who do not request testing for the purposes of academic accommodations. Lastly, additional research needs to be conducted that examines the outcome of individuals with ADHD who pursue postsecondary education. Factors contributing to academic success, especially successful completion of a degree, may hold great clinical promise. 


\section{References}

Adler, L., Barkley, R., Wilens, T., \& Ginsberg, D. (2006). Differential diagnosis of attention-deficit/hyperactivity disorder and comorbid conditions. Primary Psychiatry, 13(5), 1-14.

Advokat, C., Martino, L., Hill, B.D., \& Gouvier, W. (2007). Continuous performance test (CPT) of college students with ADHD, psychiatric disorders, cognitive deficits, or no diagnosis. Journal of Attention Disorders, 10(3), 253-256.

American Psychiatric Association. (2000). Diagnostic and Statistical Manual of Mental Disorders ( $4^{\text {th }}$ ed., Text Revision). Washington, DC: Author.

Americans with Disabilities Act of 1990, P.L. 101-336, 42 U.S.C. § 12101 et seq.

Applegate, B., Lahey, B.B., Hart, E.L., Biederman, J., Hynd, G.W., Barkley, R.A., et al. (1997). Validity of the age-of-onset criterion for ADHD: a report from the DSM IV field trials. Journal of the American Academy of Child and Adolescent Psychiatry, 36(9), 1211-1221.

Armstrong, C.L., Hayes, K.M., \& Martin, R. (2001). Neurocognitive problems in attention deficit disorder: Alternative concepts and evidence for impairment in inhibition of selective attention. Annals of the New York Academy of Sciences, 931, 196-215.

Asherson, P. (2005). Clinical assessment and treatment of attention deficit hyperactivity disorder in adults. Expert Review of Neurotherapeutics, 5(4), 525-539.

Barkley, R. (2002). Major life activity and health outcomes associated with attention deficit/hyperactivity disorder. Journal of Clinical Psychiatry, 63(12), 10-15. 
Barkley, R. (2006). Attention-Deficit Hyperactivity Disorder: A Handbook for Diagnosis and Treatment (3rd ed.). New York, NY: The Guilford Press.

Barkley, R.A. \& Biederman, J. (1997). Toward a broader definition of the age-of-onset criterion for attention-deficit hyperactivity disorder. Journal of the American Academy Of Child and Adolescent Psychiatry, 36(9), 1204-1210.

Baron, I.S. (2004). Delis-Kaplan Executive Function System. Child Neuropsychology, $10(2), 147-152$.

Biederman, J., Faraone, S., Milberger, S., Curtis, S., Chen, L., Marrs, A., et al. (1996). Predictors of persistence and remission of ADHD into adolescence: results from a four-year prospective follow-up study. Journal of the American Academy of Child and Adolescent Psychiatry, 35(3), 343-351.

Biederman, J., Faraone, S.V., Spencer, T.J., Mick, E., Monuteaux, M.C., \& Aleardi, M. (2006). Functional impairments in adults with self-reports of diagnosed ADHD: A controlled study of 1001 adults in the community. The Journal of Clinical Psychiatry, 67(4), 524-540.

Biederman, J., Farone, S.V., Spencer, T., Wilens, T., Norman, D., Lapey, K.A., et al. (1993). Patterns of psychiatric comorbidity, cognition, and psychosocial functioning in adults with attention deficit hyperactivity disorder. American Journal of Psychiatry, 150, 1792-1798.

Biederman, J., Spencer, T., \& Wilens, T. (2004). Evidence-based pharmacotherapy for attention-deficit hyperactivity disorder. The International Journal of Neuropsychopharmacology, 7(1), 77-97.

Boonstra, A.M., Oosterlaan, J., Sergeant, J.A., \& Buitelaar, J.K. (2005). Executive 
functioning in adult ADHD: a meta-analytic review. Psychological Medicine, 35, $1097-1108$.

Bridgett, D.J. \& Walker, M.E. (2006). Intellectual functioning in adults with ADHD: A meta-analytic examination of full scale IQ differences between adults with and without ADHD. Psychological Assessment, 18(1), 1-14.

Brod, M., Perwien, A., Adler, L., Spencer, T., \& Johnston, J. (2005). Conceptualization and assessment of quality of life for adults with attention-deficit/hyperactivity disorder. Primary Psychiatry, 12(6), 58-64.

Carlson, C.L. \& Mann, M. (2000). Attention deficit hyperactivity disorder, predominantly inattentive subtype. Child and Adolescent Psychiatric Clinics of North America, 9, 499-510.

Carlson, C.L. \& Mann, M. (2002). Sluggish cognitive tempo predicts a different pattern of impairment in the attention deficit hyperactivity disorder, predominantly inattentive type. Journal of Clinical Child and Adolescent Psychology, 31(1), 123-129.

Carr, L.A., Nigg, J.T., \& Henderson, J.M. (2006). Attentional versus motor inhibition in adults with attention-deficit/hyperactivity disorder. Neuropsychology, 20(4), 430441.

Castellanos, F.X., Lee, P.P., Sharp, W., Jeffries, N.O., Greenstein, D.K., Clasen, L.S., et al. (2002). Developmental trajectories of brain volume abnormalities in children and adolescents with attention deficit/hyperactivity disorder. The Journal of the American Medical Association, 288(14), 1740-1748.

Castellanos, F.X. \& Tannock, R. (2002). Neuroscience of attention-deficit/hyperactivity 
disorder: the search for endophenotypes. Nature Reviews. Neuroscience, 3(8), 617-628.

Delis, D.C., Kaplan, E., \& Kramer, J.H. (2001). The Delis-Kaplan Executive Function System: Examiner's Manual. San Antonio, TX: Psychological Corporation.

Doyle, R. (2004). The history of adult attention-deficit/hyperactivity disorder. Psychiatric Clinics of North America, 27, 203-214.

Doyle, A.E., Faraone, S.V., Seidman, L.J., Willcutt, E.G., Nigg, J.T., Waldman, I.D., et al. (2005). Are endophenotypes based on measures of executive functions useful for molecular genetic studies of ADHD? Journal of Child Psychology and Psychiatry, 46(7), 778-803.

DuPaul, G.J., Schaughency, E.A., Weyandt, L.L., Tripp, G., Kiesner, J., Ota, K., et al. (2001). Self-report of ADHD symptoms in university students: Cross gender and cross-national prevalence. Journal of Learning Disabilities, 34(4), 370-379.

Epstein, J., Johnson, D., Varia, I., \& Conners, K. (2001). Neuropsychological assessment of response inhibition in adults with ADHD. Journal of Clinical \& Experimental Neuropsychology, 23(3), 362-371.

Faraone, S.V., Biederman, J., Lehman, B.K., Spencer, T., Norman, D., Seidman, L.J., et al. (1993). Intellectual performance and school failure in children with attention deficit hyperactivity disorder and in their siblings. Journal of Abnormal Psychology, 102(4), 616-623.

Faraone, S.V. (2004). Etiology and pathophysiology of adult attention deficit/Hyperactivity Disorder. Primary Psychiatry, 11(7), 28-40.

Faraone, S.V., Spencer, T., Aleardi, M., Pagano, C., \& Biederman J. (2004). Meta 
analysis of the efficacy of methylphenidate for treating adult attention deficit/hyperactivity disorder. Journal of Clinical Psychopharmacology, 24(1), 24-29.

Fischer, M., Barkley, R.A., Smallish, L., \& Fletcher, K. (2005). Executive functioning in hyperactive children as young adults: attention, inhibition, response perseveration, and the impact of comorbidity. Developmental Neuropsychology, 27(1), 107-33.

Fitzgerald, M. (2001). Psychopharmacological treatment of adolescent and adult attention deficit hyperactivity disorder. Irish Journal of Psychological Medicine, 18(3), 93 98.

Frazier, T.W., Youngstrom, E.A., Glutting, J.J., \& Watkins, M.W. (2007). ADHD and achievement: Meta-analysis of the child, adolescent, and adult literatures and a concomitant study with college students. Journal of Learning Disabilities, 40(1), 49-65.

Frick, P.J., Lahey, B.B., Applegate, B., Kerdyck, L., Ollendick, T., Hynd, G.W., et al. (1994). DSM-IV field trials for the disruptive behavior disorders: symptom utility estimates. Journal of the American Academy of Child and Adolescent Psychiatry, 33(4), 529-539.

Gansler, D.A., Fucetola, R., Krengel, M., Stetson, S., Zimering, R., \& Makaray, C. (1998). Are there cognitive subtypes in adult Attention Deficit/Hyperactivity Disorder? The Journal of Nervous and Mental Disease, 186, 776-781.

Gaub, M. \& Carlson, C. (1997). Behavioral characteristics of DSM-IV ADHD subtypes in a school-based population. Journal of Abnormal Child Psychology, 25, 103 111. 
Glutting, J., Sheslow, D., \& Adams, W. (2002). The College ADHD Response Evaluation (CARE). Wilmington, DE: Wide Range, Inc.

Glutting, J.J., Youngstrom, E.A., \& Watkins, M.W. (2005). ADHD and college students: exploratory and confirmatory factor structures with student and parent data. Psychological Assessment, 17(1), 44-55.

Gordon, M. (2000). College students and the diagnosis of attention deficit hyperactivity disorder. Journal of American College Health, 49(1), 46-47.

Gordon, M., Lewandowski, L., Murphy, K., \& Dempsey, K. (2002). ADA-based accommodations in higher education: a survey of clinicians about documentation requirements and diagnostic standards. Journal of Learning Disabilities, 35(4), $357-363$

Guerts, H.M., Verte, S., Ooserlaan, J., Roeyers, H., \& and Sergeant, J.A. (2005). ADHD subtypes: do they differ in their executive functioning profile. Archives of Clinical Neuropsychology, 20, 457-477.

Heiligenstein, E., Conyers, L.M., Berns, A.R., and Miller, M.A. (1998). Preliminary normative data on DSM-IV attention deficit hyperactivity disorder in college students. Journal of American College Health, 46(4), 185-188.

Heiligenstein, E., Johnston, H.F., \& Nielsen, J.K. (1996). Pemoline therapy in college students with attention deficit hyperactivity disorder: a retrospective study. Journal Of American College Health, 45(1), 35-39.

Heiligenstein, E., Guenther, G., Levy, A., Savino, F., \& Fulviler, J. (1999). Psychological and academic functioning in college students with attention deficit hyperactivity disorder. Journal of American College Health, 47(4), 181-185. 
Heiligenstein, E. \& Keeling, R.P. (1995). Presentation of unrecognized attention deficit hyperactivity disorder in college students. Journal of American College Health, 43(5), 226-228.

Henderson, C. (1999). College Freshman with Disabilities, 1999: A Biennial Statistical Profile. Statistical Year 1998. (ED436900). American Council on Education, Washington, DC. HEATH Resource Center. Department of Education, Washington, DC.

Hervey, A.S., Epstein, J.N., \& Curry, J.F. (2004). The neuropsychology of adults with attention-deficit/hyperactivity disorder: A meta-analytic review. Neuropsychology, 18(3), 485-503.

Hesslinger, B., Tebartz van Elst, L., Thiel, T., Haegele, K., Hennig, J., \& Ebert, D. (2002). Frontoorbital volume reductions in adult patients with attention deficit hyperactivity disorder. Neuroscience Letters, 328(3), 319-321.

Hinshaw, S.P., Carte, E.T., Fan, C., Jassy, J.S., Owens, E.B. (2007). Neuropsychological functioning of girls with attention-deficit/hyperactivity disorder followed prospectively into adolescence: Evidence for continuing deficits? Neuropsychology, 21(2), 263-273.

Homack, S., Lee, D., \& Riccio, C.A. (2005). Test review: Delis-Kaplan executive function system. Journal of Clinical and Experimental Neuropsychology, 27(5), 599-609.

Kessler, R.C. (2006). The prevalence and correlates of adult ADHD in the United States: results from the National Comorbidity Survey Replication. The American Journal of Psychiatry, 163(4), 716-23. 
Kovner, R., Budman, C., Yitzchak, F., Sison, C., Lesser, M., \& Halperin, J. (1998). Neuropsychological testing in adult attention deficit hyperactivity disorder: A pilot study. International Journal of Neuroscience, 96, 225-235.

Lezak, M.D. (2002). Responsive assessment and the freedom to think for ourselves. Rehabilitation Psychology, 47(3), 339-353.

McBurnett, K., Pfiffner, L.J., \& Frick, P.J. (2001). Symptom properties as a function of ADHD type: an argument for continued study of sluggish cognitive tempo. Journal of Abnormal Child Psychology, 29(3), 207-213.

McLean, A., Dowson, J., Toone, B., Young, S., Bazanis, E., Robbins, T.W., \& Sahakian, B.J. (2004). Characteristic neurocognitive profile associated with adult attention deficit/hyperactivity disorder. Psychological Medicine, 34(4), 681-692.

Mehta, M.A., Goodyer, I.M., \& Sahakian, B.J. (2004). Methylphenidate improves working memory and set-shifting in AD/HD: relationships to baseline memory capacity. Journal of Child Psychology and Psychiatry and Allied Disciplines, 45(2), 293-305.

Mick, E., Faraone, S.V., \& Biederman, J. (2004). Age-dependent expression of attention deficit/hyperactivity disorder symptoms. The Psychiatric Clinics of North America, 27(2), 215-224.

Mick, E., Faraone, S.V., Biederman, J., \& Spencer, T.J. (2004). The course and outcome of attention deficit/hyperactivity disorder. Primary Psychiatry, 11(7), 42-48.

Milich, R., Balentine, A.C., \& Lynam, D.R. (2001). ADHD combined type and ADHD predominantly inattentive type are distinct and unrelated disorders. Clinical Psychology: Science and Practice, 8(4), 463-488. 
Millstein, R.B., Wilens, T.E., Biederman, J., \& Spencer, T.J. (1997). Presenting ADHD symptoms and subtypes in clinically referred adults with ADHD. Journal of Attention Disorders, 2(3), 159-166.

Murphy, K. \& Barkley, R.A. (1996). Prevalence of DSM-IV symptoms of ADHD in adult licensed drivers: Implications for clinical diagnosis. Journal of Attention Disorders, 1(3), 147-161.

Murphy, K.R., Barkley, R.A., \& Bush, T. (2001). Executive functioning and olfactory identification in young adults with attention deficit-hyperactivity disorder. Neuropsychology, 15(2), 211-220.

Murphy, K.R., Barkley, R.A., \& Bush, T. (2002). Young adults with attention deficit hyperactivity disorder: subtype differences in comorbidity, educational, and clinical history. The Journal of Nervous and Mental Disease, 190(3), 147-157.

Murphy, P. (2002). Cognitive functioning in adults with attention-deficit/hyperactivity disorder. Journal of Attention Disorders, 5(4), 203-209.

Nigg, J.T. (2006). What Causes ADHD? Understanding What Goes Wrong and Why. The Guilford Press, New York, NY.

Nigg, J.T., Blaskey, L.G., Huang-Pollock, C.L., \& Rappley, M.D. (2002). Neuropsychological executive functions and DSM-IV ADHD subtypes. Journal of the American Academy of Child Adolescent Psychiatry, 41(1), 59-66.

Nigg, J.T., Stavro, G., Ettenhofer, E., Hambrick, D.Z., Miller, T., \& Henderson, J.M. (2005). Executive functions and ADHD in adults: Evidence for selective effects on ADHD symptoms domains. Journal of Abnormal Psychology, 114(3), 706717. 
Pennington, B.F. \& Ozonoff, S. (1996). Executive functions and developmental psychopathology. Journal of Child Psychology and Psychiatry, 37(1), 51-87.

Raggi, V.L. \& Chronis, A.M. (2006). Interventions to address the academic impairment of children and adolescents with ADHD. Clinical Child and Family Psychology Review, 9(2), 85-111.

Ramsay, J.R. \& Rostain, A.L. (2006). Cognitive behavior therapy for college students with attention-deficit/hyperactivity disorder. Journal of College Student Psychotherapy, 21(1), 3-19.

Richard, M.M. (1995). Pathways to success for the college student with ADD: Accommodations and Preferred Practices. Journal of Postsecondary Education and Disability, 11, 16-30.

Safren, S.A., Sprich, S.S., Chulvick, S., \& Otto, M.W. (2004). Psychosocial treatments for adults with attention-deficit/hyperactivity disorder. Psychiatric Clinics of North America, 27, 349-360.

Sagvolden, T., Aase, H., Zeiner, P., \& Berger, D. (1998). Altered reinforcement mechanisms in attention-deficit/hyperactivity disorder. Behavioural Brain Research, 94(1), 61-71.

Sagvolden, T., Johansen, E.B., Aase, H., Russell, V.A. (2005). A dynamic developmental theory of attention-deficit/hyperactivity disorder (ADHD) predominantly hyperactive/impulsive and combined subtypes. The Behavioral and Brain Sciences, 28(3), 397-419.

Sandford, J.A. \& Turner, A. (2000). Manual for the Integrated Visual and Auditory Continuous Performance Test. Richmond, VA, Braintrain. 
Sattler, J.M. (2001). Assessment of Children: Cognitive Applications-Fourth Edition. San Diego: Jerome Sattler Publishing.

Schmitz, M., Cadore, L., Paczko, M., Kipper, L., Chaves, M., Rohde, L.A., et al. (2002). Neuropsychological performance in DSM-IV ADHD subtypes: an exploratory study with untreated adolescents. Canadian Journal of Psychiatry, 47(9), 863869.

Schoechlin, C. \& Engel, R. (2005). Neuropsychological performance in adult attention deficit hyperactivity disorder: Meta-analysis of empirical data. Archives of Clinical Neuropsychology, 20(6), 727-744.

Schweitzer, J.B., Hanford, R.B., \& Medoff, D.R. (2006, December 15). Working memory deficits in adults with ADHD: is there evidence for subtype differences? Behavioral and Brain Functions, 2, 43-53. Retrieved July 06, 2007, from http://www.behavioralandbrainfunctions.com/content/pdf/1744-9081-2-43.pdf.

Schweitzer, J.B., Lee, D.O., Hanford, R.B., Zink, C.F., Ely, T.D., Tagamets, M.A., et al. (2004). Effect of methylphenidate on executive functioning in adults with attention-deficit/hyperactivity disorder: normalization of behavior but not related brain activity. Biological Psychiatry, 56(8), 597-606.

Seidman, L.J., Valera, E.M., \& Bush, G. (2004). Brain function and structure in adults with attention-deficit/hyperactivity disorder. Psychiatric Clinics of North America, 27, 323-347.

Shaw-Zirt, B., Popali-Lehane, L., Chaplin, W., \& Bergman, A. (2005). Adjustment, social skills, and self-esteem in college students with symptoms of ADHD. Journal Of Attention Disorders, 8(3), 109-120. 
Solanto, M.V., Abikoff, H., Sonuga-Barke, E., Schachar, R., Logan, G.D., Wigal, T., et al. (2001). The ecological validity of delay aversion and response inhibition as measures of impulsivity in AD/HD: a supplement to the NIMH multimodal treatment study of AD/HD. Journal of Abnormal Child Psychology, 29(3), 215 228.

Sonuga-Barke, E.J.S. (2002). Psychological heterogeneity in AD/HD - a dual pathway model of behaviour and cognition. Behavioural Brain Research, 130, 29-36.

Sonuga-Barke, E.J.S. (2005). Causal models of attention-deficit/hyperactivity disorder: from common simple deficits to multiple developmental pathways. Biological Psychiatry, 57, 1231-1238.

Spencer, T.J., Biederman, J., \& Mick, E. (2007). Attention-deficit/hyperactivity disorder: Diagnosis, lifespan, comorbidities, and neurobiology. Ambulatory Pediatrics, 7(18), 73-81.

Stavro, G.M., Ettenhofer, M.L., \& Nigg, J.T. (2007) Executive functions and adaptive functioning in young adult attention-deficit/hyperactivity disorder. Journal of the International Neuropsychological Society, 13, 324-334.

Todd, R.D., Rasmussen, E.R., Wood, C., Levy, F., \& Hay, D.A. (2004). Should sluggish cognitive tempo symptoms be included in the diagnosis of attention deficit/hyperactivity disorder? Journal of the American Academy of Child Adolescent Psychiatry, 43(5), 588-597.

Torgersen, T., Gjervan, B., \& Rasmussen, K. (2006). ADHD in adults: A study of clinical characteristics, impairment and comorbidity. Nordic Journal of Psychiatry, 60(1), $38-43$ 
Turner, D.C. (2005). Neurocognitive effects of methylphenidate in adult attention deficit/hyperactivity disorder. Psychopharmacology, 178(2-3), 286-295.

Wadsworth, J. \& Harper, D. (2007). Adults with attention-deficit/hyperactivity disorder: Assessment and treatment strategies. Journal of Counseling and Development, 85(1), 101-108.

Walker, A.J., Shores, E.A., Trollor, J.N., Lee, T., \& Sachdev, P.S. (2000).

Neuropsychological functioning of adults with attention deficit hyperactivity disorder. Journal of Clinical and Experimental Neuropsychology, 22(1), 115-124.

Wechsler, D. (1997). Manual for the Wechsler Adult Intelligence Scale - Third Edition. San Antonio, TX: The Psychological Corporation.

Weiss, M. \& Murray, C. (2003). Assessment and management of attention-deficit hyperactivity disorder in adults. Canadian Medical Association Journal, 168(6), 715-722.

Weiss, M., Murray, C., \& Weiss, G. (2002). Adults with attention-deficit/hyperactivity disorder: Current concepts. Journal of Psychiatric Practice, 8(2), 99-111.

Welsh, M.C. \& Pennington, B.F. (1988). Assessing frontal lobe functioning in children: Views from developmental psychology. Developmental Neuropsychology, 4(3), 199-230.

Wender, P.H., Wolf, L.E., \& Wasserstein, J. (2001). Adults with ADHD: an overview. Annals New York Academy of Sciences, 931, 1-16.

Weyandt, L.L. \& DuPaul, G. (2006). ADHD in college students. Journal of Attention Disorders, 10(1), 9-19.

Weyandt, L.L., Iwaszuk, W., Fulton, K., Ollerton, M., Beatty, N., Fouts, H., et al. (2003). 
The internal restlessness scale: Performance of college students with and without ADHD. Journal of Learning Disabilities, 36(4), 382-389.

Weyandt, L.L., Linterman, I., \& Rice, J.A. (1995). Reported prevalence of attentional difficulties in a general sample of college students. Journal of Psychopathology and Behavioral Assessment, 17(3), 293-304.

Weyandt, L.L., Mitzlaff, L., \& Thomas, L. (2002). The relationship between intelligence and performance on the test of variables of attention (TOVA). Journal of Learning Disabilities, 35(2), 114-120.

Weyandt, L.L., Rice, J.A., Linterman, I., Mitzlaff, L., \& Emert, E. (1998). Neuropsychological performance of a sample of adults with ADHD, developmental reading disorder, and controls. Developmental Neuropsychology, 14, 643-656.

Wilens, T. (2003). Drug therapy for adults with attention-deficit hyperactivity disorder. Drugs, 63(22), 2395-2411.

Wilens, T.E., Biederman, J., \& Spencer, T.J. (2002). Attention deficit/hyperactivity disorder across the lifespan. Annual Review of Medicine, 53, 113-131.

Willcutt, E.G., Doyle, A.E., Nigg, J.T., Faraone, S.V., \& Pennington, B.F. (2005). Validity of the executive function theory of attention deficit hyperactivity disorder: A meta-analytic review. Biological Psychiatry, 57, 1336-1346.

Wolf, L.E. (2001). College students with ADHD and other hidden disabilities. Outcomes and interventions. Annals of the New York Academy of Sciences, 931, 385-395.

Wolf, L.E. \& Wasserstein, J. (2001). Adult ADHD: Concluding thoughts. Annals of the New York Academy of Sciences, 931, 396-408. 
Woods, S.P., Lovejoy, D.W., \& Ball, J.D. (2002). Neuropsychological characteristics of adults with ADHD: A comprehensive review of initial studies. The Clinical Neuropsychologist, 16(1), 12-34.

Woods, S.P., Lovejoy, D.W., Stutts, M.L., Ball, J.D., \& Fals-Stewart, W. (2002).

Comparative efficiency of a discrepancy analysis for the classification of attention-deficit/hyperactivity disorder in adults. Archives of Clinical Neuropsychology, 17, 351-369.

Young, S. \& Toone, B. (2000). Attention deficit hyperactivity disorder in adults: clinical issues. A report from the first NHS clinic in the UK. Counselling Psychology Quarterly, 13(3), 313-319.

Young, S., Toone, B., \& Tyson, C. (2003). Comorbidity and psychosocial profile of adults with attention deficit hyperactivity disorder. Personality and Individual Differences, 35(4), 743-755. 
Appendix A.

ADHD/LD Assessment Screening Questionnaire 
ADHD/LD Assessment Screening Questionnaire

Full Name:

Please describe current academic difficulties, as well as a brief history of the problem.

\section{$\underline{\text { SECTION 1. - Attention and Concentration }}$}

1. How is your attention and concentration?

2. Please check all of the following that apply:

I am often easily distracted

I am often forgetful in daily activities

I often fail to give close attention to details

I often make careless mistakes in schoolwork, work, or other activities

People tell me that it does not appear that I'm listening when spoken to

I often have trouble following through and completing tasks

I often have difficulty organizing tasks and activities

I tend to avoid or dislike tasks that require continuous mental effort over a period of time.

I often lose things necessary for tasks or activities (e.g., car keys, school assignment)

I am fidgety

I frequently have difficulty sitting still

I often feel restless

I tend to talk excessively

I sometimes "blurt out" answers before questions have been completed

I often have difficulty awaiting my turn

I often interrupt others' conversations or activities

SECTION 2. - Reading 
1. Are you aware of any difficulties with reading? If so, please explain:

2. With regard to reading, please check all of the following that apply:

I am a slow reader.

I often must read and re-read text before it sinks in.

I often have difficulty understanding what I've read.

I tend to mix up letters when reading.

I often think I've read a word that is different from what was actually written.

I tend to miss or overlook words.

I have difficulty keeping track of my place on the page.

I get frustrated when reading.

$\square$ I have a hard time locating the main idea of what I read.

3. Any other reading-related difficulties:

\section{SECTION 3. - Mathematics}

4. Are you aware of any difficulties with mathematics? If so, please explain:

5. With regard to mathematics, please check all of the following that apply:

I am slow with math.

$\square$ I have difficulty doing math in my head.

$\square$ I often have trouble understanding new math concepts and ideas.

$\square$ I tend to mix up numbers.

$\square$ I make careless mistakes when doing math.

$\square$ I struggle with using formulas.

$\square$ I struggle with memorizing formulas.

$\checkmark$ I have difficulty keeping track of my place when doing math problems. 
I often have difficulty sequencing the steps of a math problem.

6. Do/did you have problems with basic math skills, such as:

\author{
Addition \\ Multiplication \\ Time \\ Balancing a checkbook \\ Measurement
}

$\square$ Geometry

$\square$ Subtraction

$\checkmark$ Money $\square$ Division

7. Any other mathematics-related difficulties:

\section{SECTION 4. - Written Expression}

1. Are you aware of any difficulties with writing? If so, please explain:

2. With regard to writing, please check all of the following that apply:

I am not a very good speller.

I have difficulty organizing my thoughts when writing.

I often have difficulty putting my thoughts onto paper.

I tend to mix up words when writing or sometimes forget to include words.

I often make grammatical and punctuation errors.

I am a slow writer.

My handwriting is not as clear/legible as I would like.

When writing, I tend to wander from the main point or focus.

3. Any other writing-related difficulties: 


\section{SECTION 5. - Other Concerns}

1. Please check all of the following that apply:

I have difficulty remembering things.

I have visual problems that affect my learning.

I have auditory or hearing problems that affect my learning.

$\square$ I tend to mix up my left from my right.

2. Any other difficulties you feel might be important to mention: 
Appendix B.

ADHD/LD Testing Background Information Form. 
ADHD/LD Testing Background Information Form

Full Name

Age Date of Birth

May we contact you at the number listed?

Who referred you for testing?

Current Status

Marital Status:

Academic Status:
Today's Date Phone \# 
Have you ever been held back or failed a year of school?

If yes what grades?

Have you ever skipped a year of school?

If yes what grades?

Have you ever been evaluated for learning problems before?

If yes when?

Was a diagnosis made? What diagnosis?

Have you ever received accommodations, special services, or been placed in a resource room?

If yes what grades and what services?

Have you ever received individual tutoring?

If yes what grades?

What subjects?

Please list any courses you have failed and what year/grade.

Medical / Developmental History

General Physical Health

Hand Preference

Do you wear corrective lenses?

$\begin{array}{ll}\text { Excellent } & \text { Good } \\ \text { Left } & \text { Right } \\ \text { Yes } & \text { No }\end{array}$

Do you require a hearing aid or have any hearing impairment? Yes No

Have you received regular check-ups, eye exams, and hearing exams? Yes No

Are you currently under a physician's care?

Yes No

If yes, explain:

Do you currently take any prescription medications?

If yes, please list:

Are you currently receiving any counseling services?

Have you received counseling services in the past?

Yes

Yes

No

Yes

No

Describe any prenatal complications, or complications in your birth or infancy:

Describe any thing unusual or notable in the achievement of your developmental "milestones" (ex. learning to walk and talk.) 
Have you ever received speech therapy?

Yes

No

If yes, for which letter or sounds?

Please list any significant past illnesses or injuries:

Please list any chronic illnesses:

Please describe any head injuries:

Please list any allergies:

Please list any hospitalizations:

Year

Reason

Length of stay

Substance use. Please check all columns that apply:

\begin{tabular}{|l|l|l|l|l|l|}
\hline & Daily use & Weekly use & Monthly use & Never used & $\begin{array}{l}\text { Past use } \\
\text { only }\end{array}$ \\
\hline caffeine & & & & & \\
\hline nicotine & & & & & \\
\hline alcohol & & & & & \\
\hline marijuana & & & & & \\
\hline hallucinogens & & & & & \\
\hline crack/cocaine & & & & & \\
\hline inhalants & & & & & \\
\hline other drugs & & & & & \\
\hline
\end{tabular}

Have you ever experienced memory loss due to substance use? Yes No

Have you ever been cited for a DUI or public intoxication? Yes No

Has your substance use ever caused academic, social or family problems? Yes No

Family History

Please list the following information for all family members: 


\begin{tabular}{|c|c|c|c|c|}
\hline & Age & Marital Status & Occupation & $\begin{array}{l}\text { Highest grade } \\
\text { completed }\end{array}$ \\
\hline \multicolumn{5}{|l|}{ Birth Father } \\
\hline \multicolumn{5}{|c|}{ Birth Mother } \\
\hline \multicolumn{5}{|l|}{ Step-father } \\
\hline \multicolumn{5}{|c|}{ Step-Mother } \\
\hline \multicolumn{5}{|l|}{ Brother(s) } \\
\hline & & & & \\
\hline \multirow{2}{*}{\multicolumn{5}{|c|}{ Sister(s) }} \\
\hline & & & & \\
\hline & & & & \\
\hline & & & & \\
\hline \multicolumn{5}{|l|}{ Spouse } \\
\hline Child(ren) & & & & \\
\hline & & & & \\
\hline
\end{tabular}

Have any family members been diagnosed with the following:

$\begin{array}{lcc}\text { Learning disability } & \text { Yes } & \text { No } \\ \text { ADD/ADHD } & \text { Yes } & \text { No } \\ \text { Mental illness } & \text { Yes } & \text { No } \\ \text { Mental retardation } & \text { Yes } & \text { No }\end{array}$

Comments

Is there any other information that you believe is important to note?

If you have copies of previous evaluations or school records please feel free to bring them. They can be very helpful. 
Table 1

DSM-IV-TR Diagnostic Criteria for ADHD (APA, 2000)

A. Either (1) or (2):

(1) six (or more) of the following symptoms of inattention have persisted for at least 6 months to a degree that is maladaptive and inconsistent with developmental level:

\section{Inattention}

(a) often fails to give close attention to details or makes careless mistakes in schoolwork, work, or other activities

(b) often has difficulty sustaining attention in tasks or play activities

(c) often does not seem to listen when spoken to directly

(d) often does not follow through on instructions and fails to finish school work, chores, or duties in the workplace (not due to oppositional behavior or failure to understand instructions)

(e) often has difficulty organizing tasks and activities

(f) often avoids, dislikes, or is reluctant to engage in tasks that require sustained mental effort (such as schoolwork or homework)

(g) often loses things necessary for tasks or activities (e.g., toys, school assignments, pencils, books, or tools)

(h) is often easily distracted by extraneous stimuli

(i) is often forgetful in daily activities

(2) six (or more) of the following symptoms of hyperactivity-impulsivity have persisted for at least 6 months to a degree that is maladaptive and inconsistent with developmental level:

\section{Hyperactivity}

(a) often fidgets with hands or feet or squirms in seat

(b) often leaves seat in classroom or in other situations in which remaining seated is expected

(c) often runs about or climbs excessively in situations in which it is inappropriate (in adolescents or adults, may be limited to subjective feelings of restlessness)

(d) often has difficulty playing or engaging in leisure activities quietly

(e) is often "on the go" or often acts as if "driven by a motor"

(f) often talks excessively 
Impulsivity

(g) often blurts out answers before questions have been completed

(h) often has difficulty awaiting turn

(i) often interrupts or intrudes on others (e.g., butts into conversations or games)

A. Some hyperactive-impulsive or inattentive symptoms that caused impairment were present before age 7 years.

C. Some impairment from the symptoms is present in two or more settings (e.g., at school [or work] and at home).

D. There must be clear evidence of clinically significant impairment in social, academic, or occupational functioning.

E. The symptoms do not occur exclusively during the course of a Pervasive Developmental Disorder, Schizophrenia, or other Psychotic Disorder and are not better accounted for by another mental disorder (e.g., Mood Disorder, Anxiety Disorder, Dissociative Disorders, or a Personality Disorder).

Code based on type:

314.01 Attention-Deficit/Hyperactivity Disorder, Combined Type: if both Criteria $\mathrm{A} 1$ and $\mathrm{A} 2$ are met for the past 6 months 314.00 Attention-Deficit/Hyperactivity Disorder, Predominantly Inattentive Type: if Criterion A1 is met but Criterion A2 is not met for the past 6 months 314.01 Attention-Deficit/Hyperactivity Disorder, Predominantly HyperactiveImpulsive Type: if Criterion A2 is met but Criterion A1 is not met for the past 6 months

Coding note: For individuals (especially adolescents and adults) who currently have symptoms that no longer meet full criteria, "In Partial Remission" should be specified. 
Table 2

Demographics of $A D H D$ and $A D H D / L D$ Participants $(N=101)$

\begin{tabular}{|c|c|c|c|c|}
\hline \multirow[t]{2}{*}{ Variable } & \multicolumn{2}{|c|}{ ADHD } & \multicolumn{2}{|c|}{ ADHD/LD } \\
\hline & Frequency & Percent & Frequency & Percent \\
\hline \multicolumn{5}{|l|}{$\overline{\text { Gender }}$} \\
\hline Male & 38 & 54.3 & 18 & 58.1 \\
\hline Female & 32 & 45.7 & 13 & 41.9 \\
\hline \multicolumn{5}{|l|}{ Ethnicity } \\
\hline Caucasian/White & 60 & 85.7 & 27 & 87.1 \\
\hline African American/Black & 1 & 1.4 & 1 & 3.2 \\
\hline Biracial & 2 & 2.9 & 0 & 0 \\
\hline Asian & 3 & 4.3 & 0 & 0 \\
\hline Hispanic/Latino & 4 & 5.7 & 3 & 9.7 \\
\hline \multicolumn{5}{|l|}{ Year } \\
\hline Freshman & 30 & 42.9 & 9 & 29 \\
\hline Sophomore & 19 & 27.1 & 5 & 16.1 \\
\hline Junior & 9 & 12.9 & 10 & 32.3 \\
\hline Senior & 10 & 14.3 & 6 & 19.4 \\
\hline Graduate/Professional & 2 & 2.9 & 1 & 3.2 \\
\hline \multicolumn{5}{|l|}{ Age } \\
\hline $18-19$ & 40 & 57.1 & 8 & 25.8 \\
\hline $20-21$ & 17 & 24.3 & 14 & 45.2 \\
\hline $22-24$ & 9 & 12.9 & 6 & 19.4 \\
\hline $25+$ & 4 & 5.7 & 3 & 9.7 \\
\hline \multicolumn{5}{|l|}{ ADHD Subtype } \\
\hline Inattentive & 10 & 14.3 & 8 & 27.6 \\
\hline Hyperactive-Impulsive & 1 & 1.4 & 2 & 6.9 \\
\hline Combined & 47 & 67.1 & 12 & 41.4 \\
\hline Not Otherwise Specified & 12 & 17.1 & 9 & 24.1 \\
\hline
\end{tabular}


Table 3

Descriptive Statistics of ADHD and ADHD/LD Participants $(N=101)$

\begin{tabular}{|c|c|c|c|c|c|c|}
\hline \multirow[t]{2}{*}{ Variable } & \multirow[b]{2}{*}{$\mathrm{N}$} & \multicolumn{2}{|c|}{ ADHD } & \multirow[b]{2}{*}{$\mathrm{N}$} & \multicolumn{2}{|c|}{$\mathrm{ADHD} / \mathrm{LD}$} \\
\hline & & Mean & S.D. & & Mean & S.D. \\
\hline Grade Point Average (GPA) & 42 & 2.23 & 0.84 & 22 & 2.57 & 0.8 \\
\hline High School GPA & 63 & 3.04 & 0.53 & 26 & 2.97 & 0.6 \\
\hline SAT Total Score & 44 & 1091 & 126 & 21 & 1005 & 98 \\
\hline Full Scale Intelligence Quotient & 69 & 107 & 10.3 & 31 & 107 & 8.1 \\
\hline Verbal IQ & 68 & 107 & 11.4 & 30 & 107 & 8.3 \\
\hline Performance IQ & 68 & 105 & 11.0 & 30 & 106 & 10.3 \\
\hline \multicolumn{7}{|l|}{ CARE-SRI } \\
\hline \multicolumn{7}{|l|}{ Factor Scales } \\
\hline Inattention & 70 & 94.7 & 11.3 & 30 & 96.2 & 5.5 \\
\hline Hyperactivity & 70 & 88.8 & 17.0 & 30 & 87.4 & 16.9 \\
\hline Impulsivity & 70 & 85.2 & 18.3 & 28 & 84.5 & 17.4 \\
\hline Total & 70 & 93.4 & 12.9 & 30 & 94.8 & 8.5 \\
\hline \multicolumn{7}{|l|}{ DSM-IV Scales } \\
\hline Inattention & 70 & 94.9 & 11.3 & 30 & 96.4 & 8.9 \\
\hline Hyperactivity & 70 & 87.3 & 17.0 & 30 & 86.0 & 16.6 \\
\hline \multicolumn{7}{|l|}{ CARE-PRI } \\
\hline \multicolumn{7}{|l|}{ Factor Scales } \\
\hline Inattention & 59 & 86.4 & 14.8 & 25 & 86.1 & 15.1 \\
\hline Hyperactivity & 59 & 91.0 & 15.0 & 25 & 92.5 & 13.4 \\
\hline Total & 59 & 92.9 & 13.1 & 25 & 93.6 & 13.5 \\
\hline \multicolumn{7}{|l|}{ DSM-IV Scales } \\
\hline Inattention & 59 & 91.2 & 17.5 & 25 & 94.2 & 11.4 \\
\hline Hyperactive Impulsive & 59 & 81.6 & 18.9 & 25 & 81.0 & 18.9 \\
\hline
\end{tabular}


Table 4

Research Question 1: Cognitive Functioning of Students with ADHD (N=70)

Cognitive Functioning of Students with ADHD compared to the WAIS Standardization Sample $($ Mean $=100, \mathrm{SD}=15)$ :

$\mathrm{N} \quad$ Mean Standard Deviation $t \quad$ df $\quad$ Sig. (2-tailed)

\begin{tabular}{llrrrrl}
\hline VCI & 68 & 108.99 & 12.100 & 6.124 & 67 & $.000^{*}$ \\
POI & 68 & 109.18 & 13.090 & 5.781 & 67 & $.000^{*}$ \\
WMI & 67 & 102.37 & 11.826 & 1.643 & 66 & .105 \\
PSI & 67 & 96.78 & 11.633 & -2.268 & 66 & .027
\end{tabular}

$* \mathrm{p}<.000$ 
Table 5

Research Question 2: Attention and Response Control of Students with ADHD (N=70)

Attention and Response Control of Students with ADHD compared to the IVA Standardization Sample $($ Mean $=100, \mathrm{SD}=15)$ :

N Mean Std. Dev. $t \quad$ df $\quad$ Sig. (2-tailed)

$\begin{array}{lllllll}\text { FSRCQ } & 70 & 68.41 & 24.422 & -10.821 & 69 & .000^{*} \\ \text { FSAQ } & 70 & 57.70 & 32.917 & -10.752 & 69 & .000^{*}\end{array}$

$* \mathrm{p}<.000$ 
Table 6

Research Question 3: WAIS-III Index Scores of Students with ADHD $(\mathrm{N}=70)$ and ADHD/LD (N=31)

Cognitive Functioning of Students with ADHD Compared to Students with ADHD/LD:

$$
\mathrm{F} \quad d f \quad \mathrm{p} \quad \eta^{2} \quad \text { Power }
$$

WAIS Index Scores

$\begin{array}{lll}557 \quad 3 & .694\end{array}$

$.024 \quad .180$


Table 7

Research Question 4: Attention and Response Control of Students with ADHD (N=70) and $\mathrm{ADHD} / \mathrm{LD}(\mathrm{N}=31)$

Attention and Response Control of Students with ADHD Compared to Students with ADHD/LD:

$$
\mathrm{F} \quad d f \quad \mathrm{p} \quad \eta^{2} \quad \text { Power }
$$

$\begin{array}{llllllll}\text { IVA Full Scale Scores } & & .346 & 1 & .708 & .007 & .104\end{array}$ 
Table 8

Research Question 5: Index Score Discrepancy (N=69)

Discrepancies among WAIS-III Index Scores for Students with ADHD as Compared to the Estimated Base Rates of Such Differences Occurring in the WAIS-III Standardization Sample:

\begin{tabular}{lll} 
& $\mathrm{N}(\%$ Statistically Significant $)$ & $\mathrm{N}(\%$ Unusually Different $)$ \\
\hline VCI/POI & $32(45.71 \%)$ & $23(32.86 \%)$ \\
VCI/WMI & $38(54.29 \%)$ & $26(37.14 \%)$ \\
VCI/PSI & $35(50.00 \%)$ & $27(38.57 \%)$ \\
POI/WMI & $31(44.29 \%)$ & $21(30.00 \%)$ \\
POI/PSI & $39(55.71 \%)$ & $37(52.86 \%)$ \\
WMI/PSI & $31(44.29 \%)$ & $26(37.14 \%)$ \\
\end{tabular}


Table 9

Research Question 6: Deficits in Executive Functioning as measured by the D-KEFS $(\mathrm{N}=70)$

Executive Functions

Descriptive Statistics

Below Average

Frequency $(\%)$

Cognitive Flexibility

TMT Number-Letter Switching $\quad 7 \quad$ (10.00)

Verbal Fluency, Category Switching 6

Design Fluency, Switching 5

Color Word, Inhibition/Switching $\quad 8 \quad$ (11.43)

Verbal Fluency

Verbal Fluency, Letter Fluency $14 \quad$ (20.00)

Verbal Fluency, Category Fluency $\quad 8$ (11.43)

Non-Verbal Fluency

Design Fluency, Filled Dots 6

Design Fluency, Empty Dots Only 6

Concept Formation

Sorting, Free Sorting Confirmed Sorts

Sorting, Free Sorting Description 2

Sorting, Sort Recognition Description 12

Twenty Questions, Initial Abstraction $\quad 6 \quad$ (8.57)

Proverb Test, Total Achievement $\quad 5 \quad$ (7.14)

Word Context, Consecutively Correct 6

Problem Solving

Twenty Questions, Total Achievement 3

Planning

Tower Test, Total Achievement $\quad 9$ 


\section{Supplemental Tables}

Table 10

Strengths in Executive Functioning as measured by the D-KEFS $(\mathrm{N}=70)$

Executive Functions

$$
\text { Descriptive Statistics }
$$

Below Average Above Average

Frequency $(\%) \quad$ Frequency $(\%)$

Cognitive Flexibility

TMT Number-Letter Switching

Verbal Fluency, Category Switching

Design Fluency, Switching

Color Word, Inhibition/Switching

$\begin{array}{llll}7 & (10.00) & 0 & (0.00) \\ 6 & (8.57) & 8 & (11.43) \\ 5 & (7.14) & 8 & (11.43) \\ 8 & (11.43) & 5 & (7.14)\end{array}$

Verbal Fluency

Verbal Fluency, Letter Fluency

Verbal Fluency, Category Fluency

$\begin{array}{rrr}14 & (20.00) & 22 \\ 8 & (11.43) & 25\end{array}$

Non-Verbal Fluency

Design Fluency, Filled Dots

$\begin{array}{lll}6 & (8.57) \quad 17\end{array}$

(24.29)

Design Fluency, Empty Dots Only

$6 \quad(8.57)$

$10 \quad(14.29)$

Inhibition

Color Word, Inhibition

$15 \quad(21.43) \quad 3$

Concept Formation

Sorting, Free Sorting Confirmed Sorts $\quad 1 \quad$ (1.43) $\quad 14 \quad$ (20.00)

$\begin{array}{lllll}\text { Sorting, Free Sorting Description } & 2 & (2.86) & 15 & (21.43)\end{array}$

Sorting, Sort Recognition Description $\quad 12 \quad(17.14) \quad 12 \quad$ (17.14)

Twenty Questions, Initial Abstraction $\quad 6 \quad$ (8.57) $\quad 19 \quad$ (27.14)

Proverb Test, Total Achievement $\quad 5 \quad$ (7.14) $16 \quad$ (22.86)

Word Context, Consecutively Correct $\quad 6 \quad$ (8.57) $\quad 17 \quad$ (24.29)

Problem Solving

Twenty Questions, Total Achievement $3 \quad$ (4.29)

Planning

Tower Test, Total Achievement

$9 \quad(12.86) \quad 12$

$(17.14)$ 
Table 11.

Descriptive Statistics for D-KEFS Tests of Executive Function (N=70)

Tests of Executive Function

Descriptive Statistics

\begin{tabular}{|c|c|c|}
\hline & Mean & Standard Deviation \\
\hline \multicolumn{3}{|l|}{ Trail Making Test } \\
\hline Visual Scanning & 9.07 & 3.141 \\
\hline Number Sequencing & 9.49 & 2.603 \\
\hline Letter Sequencing & 9.83 & 2.818 \\
\hline Number-Letter Switching & 9.54 & 2.495 \\
\hline Motor Speed & 10.03 & 2.787 \\
\hline \multicolumn{3}{|l|}{ Verbal Fluency } \\
\hline Letter Fluency & 10.86 & 3.633 \\
\hline Category Fluency & 11.10 & 3.056 \\
\hline Category Switching & 10.59 & 3.638 \\
\hline \multicolumn{3}{|l|}{ Design Fluency } \\
\hline Filled Dots & 10.26 & 2.758 \\
\hline Empty Dots Only & 10.29 & 2.562 \\
\hline Switching & 11.14 & 2.814 \\
\hline \multicolumn{3}{|l|}{ Color Word } \\
\hline Color Naming & 8.80 & 3.027 \\
\hline Word Reading & 9.39 & 2.704 \\
\hline Inhibition & 9.24 & 2.683 \\
\hline Inhibition/Switching & 9.20 & 3.044 \\
\hline \multicolumn{3}{|l|}{ Sorting } \\
\hline Free Sorting Confirmed Sorts & 10.74 & 1.901 \\
\hline Free Sorting Description & 10.83 & 2.014 \\
\hline Sort Recognition & 9.99 & 2.590 \\
\hline \multicolumn{3}{|l|}{ Twenty Questions } \\
\hline Initial Abstraction & 11.10 & 3.048 \\
\hline Total Weighted Achievement & 11.35 & 2.149 \\
\hline \multicolumn{3}{|l|}{ Word Context } \\
\hline Total Consecutively Correct & 10.86 & 2.164 \\
\hline \multicolumn{3}{|l|}{ Tower Test } \\
\hline Total Achievement & 10.36 & 2.466 \\
\hline \multicolumn{3}{|l|}{ Proverb Test } \\
\hline Total Achievement & 10.52 & 2.656 \\
\hline
\end{tabular}

